\title{
Modelling and Optimization Methods for a Microchannel Heat Exchanger
}

by

\section{Josh Mc Lellan}

\author{
A thesis submitted to \\ the Faculty of Graduate and Postdoctoral Affairs \\ in partial fulfilment of \\ the requirements for the degree of \\ Master of Applied Science \\ in \\ Mechanical Engineering
}

Ottawa-Carleton Institute for Mechanical and Aerospace Engineering

Department of Mechanical and Aerospace Engineering

Carleton University

Ottawa, Ontario, Canada

May 2015

Copyright (C)

2015 - Josh McLellan 
The undersigned recommend to

the Faculty of Graduate and Postdoctural Affairs

acceptance of the thesis

\title{
Modelling and Optimization Methods for a Microchannel Heat Exchanger
}

\author{
Submitted by Josh $\mathbf{M}^{\mathrm{c}}$ Lellan \\ in partial fulfilment of the requirements for the degree of \\ Master of Applied Science
}

Dr. Tarik Kaya, Supervisor

Dr. John A. Goldak, Co-Supervisor

Dr. Metin Yaras, Department Chair

Carleton University

2015 


\section{Abstract}

Microchannel heat exchangers are being considered for use in the Generation IV nuclear reactors for their ability to provide increased thermal efficiency in a small volume relative to other types of heat exchangers via an extremely high surface area-to-volume ratio. Three distinct analysis methods that may be used to evaluate the technology are presented in this work: finite element method modelling, application of smoothed particle hydrodynamics, and Kriging-based optimization. The finite element method model generated for a single pair of channels for the hot and cold working fluids yields results that agree with those produced using the effectiveness-number of transfer units method, and is a suitable base for the optimization performed. More complex free surface flows are effectively modelled using smoothed particle hydrodynamics in a number of demonstrative cases, including boiling flow through a heated channel. The results generated with this method qualitatively simulate the observed physics and match analytical solutions in problems where one exists, although further evaluation

of the VrSuite solvers must occur once additional data extraction capabilities are introduced. An initial optimization of a microchannel heat exchanger is performed using the effectiveness-number of transfer units method once more, determining an optimal design with an objective function value of 0.081 that is obtained through a combination of a $3.29 \mathrm{~m}$ long channel, $0.023 \mathrm{~m}$ channel width, a $0.0001 \mathrm{~m}$ wall thickness between channels, and hot and cold inlet flow velocity of $3.91 \mathrm{~m} / \mathrm{s}$. A preliminary optimization using finite element methods is also performed. 
To my family 


\section{Acknowledgments}

I would first like to express the most sincere gratitude to my supervisors, Dr. John Goldak and Dr. Tarik Kaya of Carleton University, for their excellent guidance and seemingly endless patience throughout this process. Furthermore, I owe a debt of gratitude to the Goldak Technologies Inc ${ }^{\circledR}$ staff, which has included Dan Downey, Stanislav Tchernov, and Jianguo Zhou, for their technical support throughout my endeavours with the VrSuite software. Their assistance has been tremendous.

I offer thanks to my colleagues Graeme Schmidt, Hossein Nimrouzi, Komeil Kazemi, and Alex Zakharchenko for their time and support. I would also like to thank Anne Wice for her companionship, for always being there to keep me balanced and focused, and for providing motivation when it was most needed. I could not have done this without you.

Finally, I would like to thank the Carleton University Department of Mechanical and Aerospace Engineering for this opportunity, and to Natural Resources Canada (NRCan) and the National Sciences and Engineering Research Council (NSERC) for their support of the Canadian Generation IV National Program. 


\section{Table of Contents}

Abstract $\quad$ iii

Acknowledgments $\quad$ v

Table of Contents $\quad$ vi

List of Tables $\quad$ ix

List of Figures $\quad$ X

List of Acronyms xii

List of Symbols $\quad$ xiv

1 Introduction 1

1.1 Background ......................... 4

1.2 Literature Review . . . . . . . . . . . . . . . . . . . . . 6

1.2.1 Compact Heat Exchanger Analyses . . . . . . . . . . . . . . . 8

1.2.2 Smoothed Particle Hydrodynamics . . . . . . . . . . . . . . 10

1.2.3 Optimization ................... . . 12

1.3 Motivation and Outline of the Research . . . . . . . . . . . . . . 14

1.3.1 Thesis Outline. . . . . . . . . . . . . 14 
2 Microchannel Heat Exchanger Analysis with FEM 17

2.1 VrSuite FEM Solvers and Governing Equations . . . . . . . . . . . 18

2.1.1 Governing Equations . . . . . . . . . . . . . . . . . 18

2.1.2 Operator Splitting and Data Flow . . . . . . . . . . . 20

2.2 Heat Exchanger Analysis - Number of Transfer Units Method . . . . 22

2.2.1 The $\epsilon$-NTU Method . . . . . . . . . . . . . . . 23

2.3 MCHX FEM Modelling . . . . . . . . . . . . . . . . . . 25

2.3.1 Initial MCHX Model - Single Channel Pair . . . . . . . . . . . 26

2.3.2 Mesh Sensitivity Analysis . . . . . . . . . . . . . . . 30

2.3.3 Model Refinement - Quarter Channel Pair . . . . . . . . . . . 32

3 Introduction to Smoothed Particle Hydrodynamics 34

3.1 What is Smoothed Particle Hydrodynamics? . . . . . . . . . . . . . . 34

3.2 VrSuite SPH Solvers and Governing Equations . . . . . . . . . . . 36

3.2.1 Governing Equations . . . . . . . . . . . . . . . . 36

3.2.2 Operator Splitting and Solver Data Flow . . . . . . . . . . . 37

3.3 Required Data Pre-Processing . . . . . . . . . . . . . . 38

3.3.1 Material Property Solver . . . . . . . . . . . . . . . . 39

3.4 Developed SPH Models . . . . . . . . . . . . . . . . . . . . . . 40

3.4.1 Free Surface Flow: Falling Drop (NS) . . . . . . . . . . . . . . 40

3.4.2 Free Surface Flow: Inlet and Outlet (NS) . . . . . . . . . . . . 42

3.4.3 Fluid Solidification: The Stefan Problem (Thermal) . . . . . . 43

3.4.4 Pool Boiling of Water (Coupled NS and Thermal) . . . . . . 45

3.4.5 Heated Channel Flow (Coupled NS and Thermal) . . . . . . . 47

3.5 Possible Model Adaptations . . . . . . . . . . . . . . . . . . . . 49

4 Optimization of the MCHX Design 50

4.1 Optimization Methods . . . . . . . . . . . . 51 
4.1.1 Local and Global Optimization . . . . . . . . . . . . . 52

4.1.2 General Requirements for Performing Optimization . . . . . . 53

4.2 VrSuite Optimization Capabilities . . . . . . . . . . . . 53

4.3 Initial MCHX Optimization - Number of Transfer Units Method . . . 58

4.3.1 Objective Function . . . . . . . . . . . . . . . . . 59

4.3.2 Design Evaluation Method . . . . . . . . . . . . . . . . 60

4.3.3 Design Space and Optimization Setup . . . . . . . . . . 63

4.3.4 Results..................... 65

4.4 Initial Expansion to Optimization using FEM . . . . . . . . . . 70

4.4.1 Choice of Design Space and Objective Function . . . . . . . . 71

4.4 .2 Results ..................... . . . 72

5 Conclusions $\quad 75$

5.1 MCHX FEM Model Development and Analysis . . . . . . . . . 76

5.2 Use of Smoothed Particle Hydrodynamics . . . . . . . . . . . . . . 78

5.3 Optimization Method for the MCHX . . . . . . . . . . . . 80

5.4 Recommendations for Future Work . . . . . . . . . . . . . . 83

$\begin{array}{lr}\text { References } & 87\end{array}$

$\begin{array}{lll}\text { Appendix A VrSuite Solver Verification } & 92\end{array}$

A.1 FEM Solvers . . . . . . . . . . . . . . . . . . . . . . 92

A.1.1 VrSuite Thermal Solver . . . . . . . . . . . . . . . . . . 92

A.2 SPH Solvers . . . . . . . . . . . . . . . . . . . 94

A.2.1 SPH Thermal Solver . . . . . . . . . . . . . . 94

A.2.2 Navier-Stokes (NS) Solver . . . . . . . . . . . . . . 96

Appendix B MCHX Optimization: VrSuite-Generated Report $\quad 97$ 


\section{List of Tables}

2.1 Mesh sensitivity analysis summary . . . . . . . . . . . . . 31

4.1 Design variable bounds for initial MCHX optimization . . . . . . . 64

4.2 Additional MCHX objective function solver parameters . . . . . . . 65

4.3 MCHX DoE matrix for Kriging iteration 5 . . . . . . . . . 66

B.1 VrSuite design variable designations for optimization report graphs . 98 


\section{List of Figures}

1.1 World net operating capacity and total number of nuclear reactors since $1954 \ldots \ldots \ldots \ldots$. . . . . . . . . . . . . . . . . 1

1.2 Lifecycle GHG emissions intensity of various power generation methods 2

1.3 Generalized temperature and pressure capabilities of the compact HX

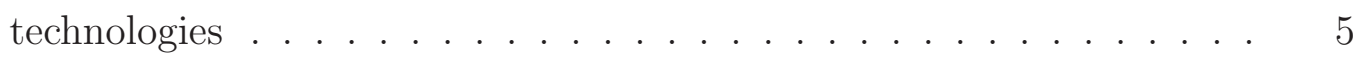

1.4 MCHX and shell-and-tube HX comparison, with micrograph . . . . 6

1.5 Stacking of chemically etched MCHX plates prior to diffusion bonding 7

1.6 Oscillations of a VdW drop modelled using $\mathrm{SPH} \ldots \ldots \ldots \ldots$

2.1 VrSuite FEM solver data flow for coupled analyses . . . . . . . . . . 21

2.2 Three levels of complexity for initial MCHX model . . . . . . . 25

2.3 Mesh used for the MCHX full channel pair model . . . . . . . . 27

2.4 Fluid and solid temperature profiles - Full channel pair . . . . . . 28

2.5 Initial MCHX FEM results normalized to the analytical values . . . . 29

2.6 Initial MCHX model mesh sensitivity plots for $\mathrm{q}_{\text {sim }}$ and $\epsilon \ldots \ldots 31$

2.7 Proposed mesh for the MCHX quarter channel pair model . . . . . . 33

3.1 Comparison of typical FEM mesh and SPH discretization . . . . . 35

3.2 Material property pre-processing code output . . . . . . . . 39

3.3 Temporal evolution of particle location for a free-falling liquid 'drop' . 41

3.4 Free surface flow with inlet and outlet . . . . . . . . . . . . . 42

3.5 Simulated results for the Stefan problem using SPH . . . . . . . . 45 
3.6 Temporal evolution of particle temperature and location in boiling pool 47

3.7 Boiling flow within a heated channel using $\mathrm{SPH} \ldots$. . . . . . . . 48

4.1 Semi-infinite bar mesh with applied BCs . . . . . . . . . . . . 54

4.2 VrSuite Kriging optimization output - Obj. fn. versus design value . 56

4.3 VrSuite Kriging optimization output - Overall obj. fn. values . . . . . 56

4.4 Resulting temperature profiles for six designs selected by Kriging op-

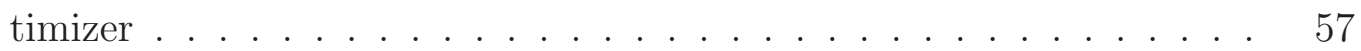

4.5 Solution procedure for MCHX modelling with $\epsilon$-NTU . . . . . . . . . 61

4.6 Convergence of MCHX optimization with $\epsilon-\mathrm{NTU}$. . . . . . . . . 65

4.7 Objective function response to MCHX design variables . . . . . . . 67

4.8 Kriging $1^{\text {st }}$ iteration results for MCHX optimization with FEM . . . . 72

4.9 Kriging $3^{\text {rd }}$ iteration results for MCHX optimization with FEM . . . 74

5.1 Possible SPH particle discretization for MCHX channel pair . . . . . 85

A.1 VrSuite FEM thermal solver verification - 1-D semi-infinite conduction

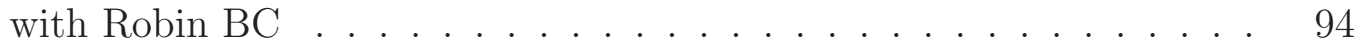

A.2 VrSuite SPH thermal solver verification - 1-D semi-infinite conduction with Dirichlet BC . . . . . . . . . . . . . . . . . 96 


\section{List of Acronyms}

\begin{tabular}{ll}
\hline Acronyms & Definition \\
\hline \hline AD & Advection-diffusion (solver) \\
BC & Boundary condition \\
CFD & Computational fluid dynamics \\
DoE & Design of experiment \\
FEM & Finite element method \\
FS & Solid fraction \\
GA & Genetic algorithm \\
Gen IV & Generation four (of nuclear reactor designs) \\
GHG & Leat exchanger \\
LC & Greenhouse gas
\end{tabular}




\begin{tabular}{ll} 
Acronyms & Definition \\
\hline \hline MCHX & Microchannel heat exchanger \\
NIST & National Institute of Standards and Technology \\
NRCan & Nuclear power plant \\
NS & Natural Resources Canada \\
NSERC & Navier-Stokes (solver) \\
NTU & Number of transfer units \\
PCHE & Printed circuit heat exchanger \\
PDE & Partial differential equation \\
PWR & Pressurized water reactor \\
SPH & Smoothed particle hydrodynamics \\
SPH-NS & Smoothed particle hydrodynamics Navier-Stokes (solver) \\
\hline
\end{tabular}




\section{Nomenclature (In order of appearance)}

\begin{tabular}{|c|c|}
\hline Symbol & Definition \\
\hline Re & Reynolds number \\
\hline$j$ & Colburn factor \\
\hline$f$ & Fanning friction factor \\
\hline$v$ & Velocity, $\left[\frac{m}{s}\right]$ \\
\hline$t$ & Time, $[s]$ \\
\hline$P$ & Pressure, $[P a]$ \\
\hline$T$ & Temperature, $[K]$ \\
\hline$k$ & Thermal conductivity, $\left[\frac{W}{m \cdot K}\right]$ \\
\hline$c$ & Specific heat capacity, $\left[\frac{\mathrm{J}}{\mathrm{kg} \cdot \mathrm{K}}\right]$ \\
\hline$q^{\prime \prime}$ & Heat flux, $\left[\frac{W}{m^{2}}\right]$ \\
\hline$Q$ & Volumetric heat generation, $\left[\frac{W}{m^{3}}\right]$ \\
\hline$b$ & Body force, $[N]$ \\
\hline$E$ & $6 \times 6$ elasticity tensor, $[\mathrm{Pa}]$ \\
\hline
\end{tabular}




\begin{tabular}{|c|c|}
\hline Symbol & Definition \\
\hline$u$ & Displacement, $[m]$ \\
\hline$q$ & Heat transfer rate, $[W]$ \\
\hline C & Heat capacity rate, $\left[\frac{J}{K \cdot s}\right]$ \\
\hline$m$ & Mass, $[k g]$ \\
\hline$H$ & Overall heat transfer coefficient, $\left[\frac{W}{m^{2} \cdot K}\right]$ \\
\hline$A$ & Heat transfer area, $\left[\mathrm{m}^{2}\right]$ \\
\hline$w$ & Width, $[m]$ \\
\hline$L$ & Length, $[m]$ \\
\hline$x$ & Location, $[m]$ \\
\hline$W$ & Gaussian interpolation function \\
\hline$r$ & Particle position, $[\mathrm{m}]$ \\
\hline$g$ & Gravity, $\left[\frac{m}{s^{2}}\right]$ \\
\hline$U$ & Internal energy, $[J]$ \\
\hline$X$ & Interpolation length $[\mathrm{m}]$ \\
\hline$B$ & Constant related to the bulk modulus of elasticity, $\left[\frac{\mathrm{kg}}{\mathrm{m} \cdot \mathrm{s}^{2}}\right]$ \\
\hline$s$ & Speed of sound, $\left[\frac{\mathrm{m}}{\mathrm{s}}\right]$ \\
\hline$h$ & Specific enthalpy, $\left[\frac{J}{k g}\right]$ \\
\hline$M$ & Matrix row count \\
\hline
\end{tabular}




\begin{tabular}{ll}
\hline Symbol & Definition \\
\hline \hline$N$ & Matrix column count \\
$N u$ & Nusselt number \\
$P r$ & Prandtl number \\
$F$ & Objective function value \\
$f$ & Darcy friction factor \\
$e$ & Surface roughness, $[m]$ \\
& \\
\hline
\end{tabular}

\section{Greek Letter Definition}

\begin{tabular}{ll}
\hline \hline$\epsilon$ & Effectiveness \\
$\rho$ & Density, $\left[\frac{\mathrm{kg}}{\mathrm{m}^{3}}\right]$ \\
$\mu$ & Dynamic viscosity, $[\mathrm{Pa} \cdot \mathrm{s}]$ \\
$\alpha$ & Thermal diffusivity, $\left[\frac{\mathrm{m}^{2}}{\mathrm{~s}}\right]$ \\
$\sigma$ & Cauchy stress tensor, $[\mathrm{Pa}]$ \\
$\varepsilon$ & Green-Lagrange strain tensor \\
$\xi$ & Viscous calibration parameter \\
$\phi$ & Small parameter
\end{tabular}




\begin{tabular}{ll} 
Greek Letter & Definition \\
\hline \hline$\gamma$ & Empirically determined equation of state constant \\
$\lambda$ & Property-dependant constant for Stefan problem \\
$\eta$ & Objective function component \\
$\beta$ & Objective function weighting factor \\
\hline
\end{tabular}

\begin{tabular}{ll}
\hline Subscript & Definition \\
\hline \hline wall & Wall value (e.g. Wall thickness) \\
$c r$ & Critical value \\
$p$ & Constant pressure \\
max & Maximum value \\
$h$ & Hot fluid value \\
$i$ & Inlet condition \\
$c$ & Cold fluid value \\
min & Minimum value \\
&
\end{tabular}




\begin{tabular}{|c|c|}
\hline Subscript & Definition \\
\hline ana & Analytical value \\
\hline solve & Solution value (e.g. Total solver run time) \\
\hline$a$ & Variable value for arbitrary particle $a$ \\
\hline$b$ & Variable value for arbitrary particle $b$ \\
\hline$a b$ & $\begin{array}{l}\text { Variable value for arbitrary particle } a \text { minus that for arbi- } \\
\text { trary particle } b\end{array}$ \\
\hline 0 & Reference or initial value \\
\hline$m$ & Melting value \\
\hline 1 & Boundary condition value (Dirichlet or Neumann) \\
\hline$S F$ & Solidification front \\
\hline$N u$ & Thermal-related objective function value \\
\hline$f$ & Friction-related objective function value \\
\hline$S m$ & Smooth surface value \\
\hline$D$ & Based on hydraulic diameter (e.g. Reynolds number) \\
\hline$b$ & Bulk value \\
\hline$h y$ & Hydraulic value (e.g. Hydraulic diameter) \\
\hline$\infty$ & Ambient value (e.g. Ambient temperature) \\
\hline
\end{tabular}




\section{Chapter 1}

\section{Introduction}

Since the first commercial nuclear power station started operation in the 1950's, there have been two distinct trends in the total net operating capacity of the world's fleet of commercial nuclear reactors. Between the infancy of the industry and the mid-1990's, there was a somewhat steady increase in operating capacity, where nuclear power's share in the world's overall power generation reached a historic peak of $17 \%$ in 1993 with a global net operating capacity of approximately 360 GWe [1].

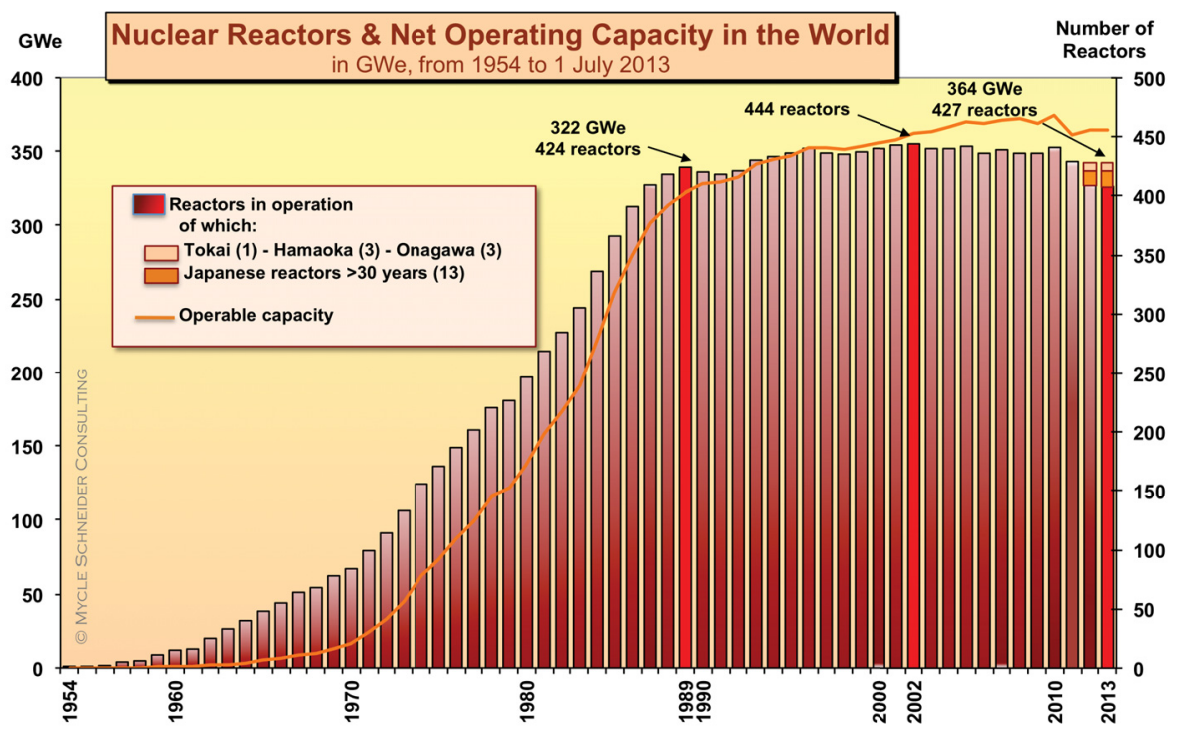

Figure 1.1: The net operating capacity and total number of nuclear reactors in the world since 1954 [1]. 
Since then, the overall net operating capacity in the world has remained fairly steady, hitting a peak of 375 GWe in 2010 and subsequently falling slightly to a current installed capacity of 371 GWe, a decline due in large part to the Fukushima accident $[1,2]$. Despite this small drop in overall operating capacity, nuclear power still holds approximately a 11\% of the world's overall power generation, and is a key contributor to meeting ever-increasing global energy demands [1]. The current estimate of the world's population is approximately 7.18 billion people, a figure that is projected to rise to nearly 9.38 billion people by the year 2050 [3]. This population growth imposes a significant increase in the demand for energy, which is expected to grow $81 \%$ from the 19,004 TWh quoted for 2011 to 34,454 TWh in the year 2035 [4]. Nuclear energy is a viable option for meeting the increased load imposed by a larger population as it "is especially suitable for large-scale, continuous electricity demands which [require] reliability (i.e. base-load), and hence ideally matched to increasing urbanisation worldwide [4]." Furthermore, nuclear power generation offers low lifecycle greenhouse gas (GHG) emissions intensity when compared with other methods of producing electricity, exhibited in Figure 1.2.

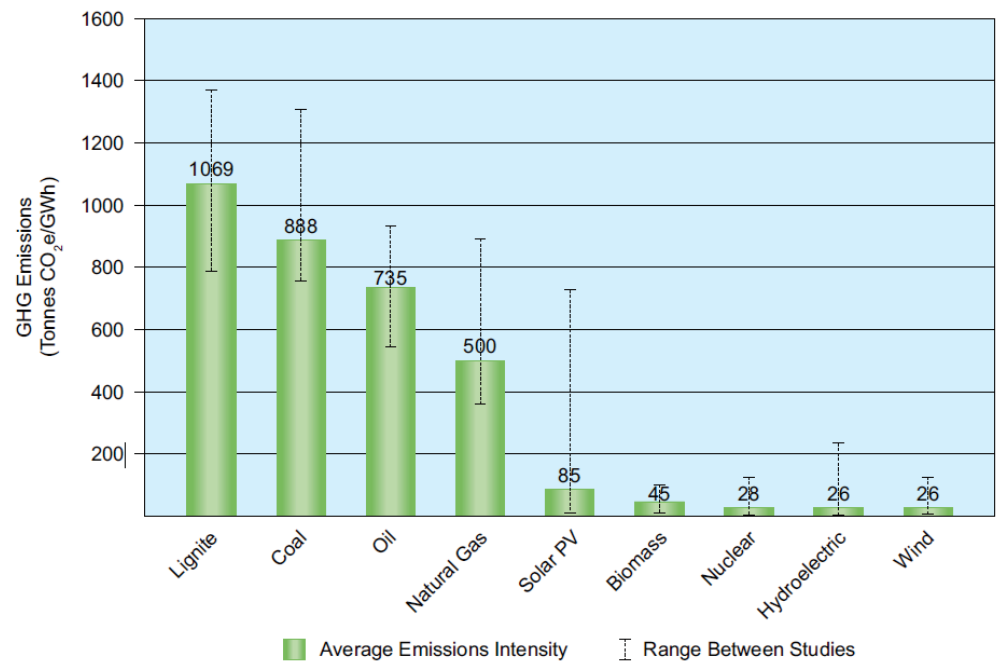

Figure 1.2: The lifecycle GHG emissions intensity of various power generation methods [5]. 
Nuclear technologies are also promising from an economic point of view. An often cited metric for evaluating the competitiveness of the various energy generation technologies is the levelized cost of electricity (LCOE), which represents "per-kilowatthour cost (in real dollars) of building and operating a generating plant over an assumed financial life and duty cycle," including factors such as capital, fuel, and operations and maintenance costs [6]. A recent projection of costs associated with plants expected to enter service in 2019 estimates nuclear power generation to have an average LCOE of 86.1 \$/MWh, a figure that is much lower than the 95.6 \$/MWh of conventional coal, 103.8 \$/MWh of advanced combustion turbine natural gas, and the 102.6 \$/MWh of biomass, but is only marginally higher than the $80.3 \$ / \mathrm{MWh}$ associated with wind energy production [6]. These figures indicate that nuclear power offers a lower cost option for alleviating the increasing energy demand of a rising global population, despite high capital and equipment costs associated with construction of a nuclear power plant (NPP) making up a significant part of the total cost. This prohibits alleviating the world's evolving energy needs by simply increasing the size of its reactor fleet, and necessitates improving the overall designs or the components used. The next generation of nuclear reactors (Gen IV) is currently being developed by a consortium of countries, including Canada and the United States of America. While focus is being placed on development of the new designs, a number of the components used in the current generation of NPPs provide an opportunity to improve the overall performance of the plant and possibly reduce the aforementioned capital and equipment costs associated with construction or operations and maintenance. A microchannel heat exchanger (MCHX) is a technology that has potential to not only reduce the capital costs associated with construction of NPPs by acting as a potential substitute for other pieces of significantly more expensive equipment, but possibly improve the overall efficiency of the plant by offering excellent heat transfer characteristics. This work serves to detail methods by which the design of an MCHX can be analyzed and 
subsequently optimized to be suitable for use in Gen IV of advanced nuclear reactor designs, beginning with a brief description of the MCHX technology and its origins.

\section{$1.1 \quad$ Background}

Development of the compact heat exchanger technology started with the initial efforts of Tuckerman and Pease in 1981 "on the cooling of very large-scale integrated circuits... where they first explained the concepts behind microchannel heat sinks and predicted that single-phase forced convective cooling in microchannels could potentially remove heat at a rate of the order of $1,000 \mathrm{~W} / \mathrm{m}^{2}$ [7]." Their work encouraged research in a new field of study for electronics cooling where channels smaller than $200 \mu \mathrm{m}$ were used in small heat exchangers (HXs). MCHXs have been predominantly used in the electronics industry since then, finding use in "applications where fluxes exceed $100 \mathrm{~W} / \mathrm{cm}^{2}$, such as high-powered microelectronics, diode array lasers, supercomputers and plasma-facing components [8]." This advancement in the MCHX technology was paralleled in the process industry, beginning with development of the printed circuit heat exchanger (PCHE), another form of compact heat exchanger very similar to an MCHX, in 1980 at the University of Sydney for refrigeration applications $[9,10]$. Heatric Ltd. capitalized on these advancements and commercialized the technology in the mid-1980s, and was eventually purchased by the Meggitt group in 1990. They have since become the leading manufacturer and developer of MCHXs.

The MCHXs created for use in the process industry today commonly utilize channels that are exceptionally small, having hydraulic diameters on the order of $1 \mathrm{~mm}$ to $3 \mathrm{~mm}$ [11]. While these dimensions aren't technically "micro" scale, the heat exchanger retains the moniker "microchannel" given the similarities in manufacturing, design, and the heat transfer principles that were initially utilized in designing the 
electronics MCHXs. A common method used to manufacture an MCHX is diffusion bonding, beginning by taking a plate of a desired material and chemically etching channels into its face. A large number of these etched plates are then pressed together and heated to near the melting temperature of the material to encourage grain growth across their interfaces, producing a monolithic piece that has the same mechanical properties as the parent material [12]. The resulting compact heat exchanger typically has much larger range of operating conditions than other types of heat exchanger, which is generalized in Figure 1.3.

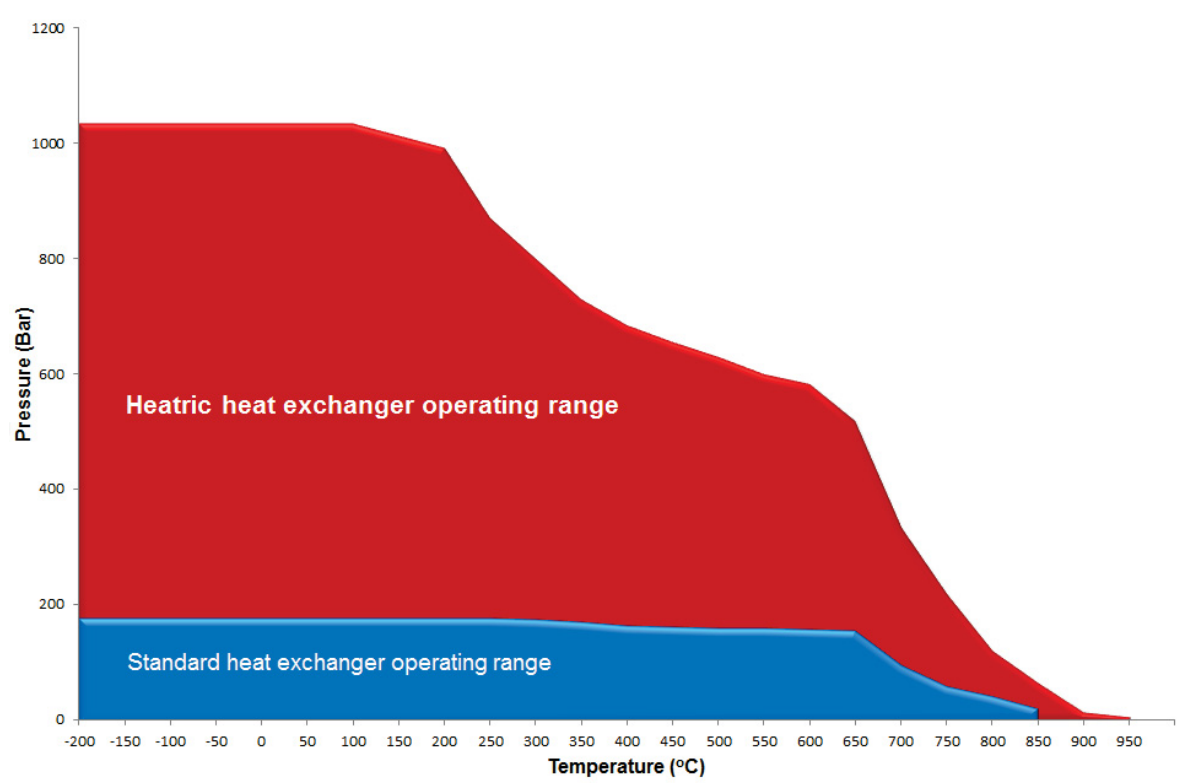

Figure 1.3: Temperature and pressure capabilities of the compact heat exchanger technology (PCHE) relative to standard heat exchangers [13].

In addition to the excellent mechanical properties yielded, the diffusion bonding process allows for the creation of an MCHX that has an exceptionally high number of small channels that is capable of transferring higher amounts of heat than a traditional shell-and-tube heat exchanger due to a large surface area-to-volume ratio for the channels, while reducing the total volume of exchanger required for an equivalent heat load. This ability to provide excellent heat transfer and a large operating 
range in a relatively small package with ease in manufacturing is a leading motive for attempting to utilize the MCHX technology in the Gen IV reactor designs. This is emphasized in Figure 1.4 when a comparison of the relative sizes of a shell-and-tube heat exchanger and an MCHX designed for an equivalent heat load and input conditions are observed. A micrograph of a pair of etched plates that have been diffusion bonded is also depicted to provide a better sense of the size of the channels.
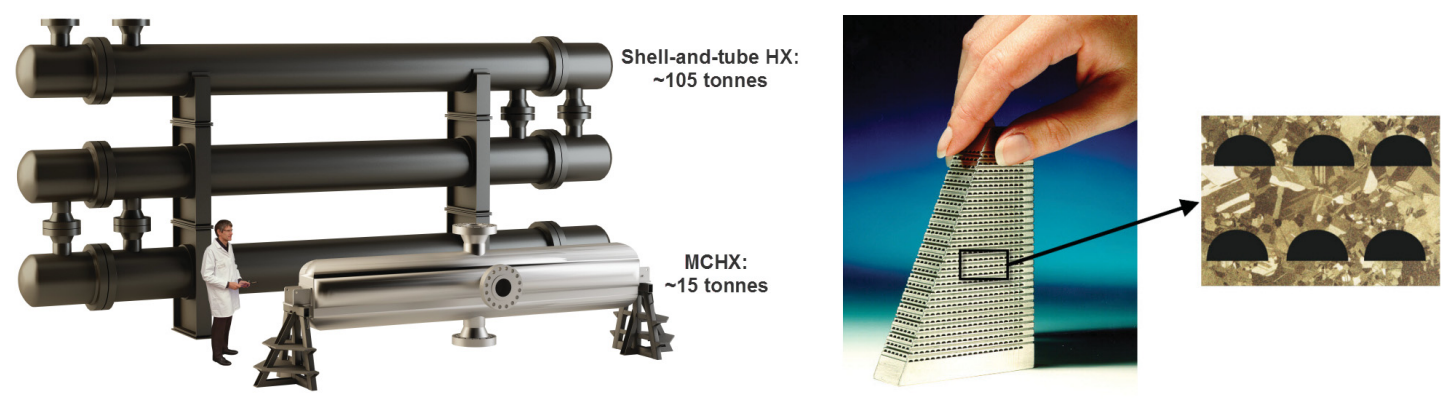

Figure 1.4: A shell-and-tube heat exchanger and equivalent MCHX (left), and sample MCHX segment with enlarged micrograph of the stacked plates (right) (Both courtesy of Heatric Ltd.)

\subsection{Literature Review}

As mentioned in Section 1.1, some of the most desirable characteristics of the microchannel heat exchanger technology are the excellent heat transfer and mechanical properties it can provide while maintaining a very small total volume. Commercially available MCHXs are known to be able to accommodate pressures upwards of $100 \mathrm{MPa}$, temperatures as high as $980^{\circ} \mathrm{C}$, and reach effectiveness values of $\epsilon=0.98$ or higher, all with a core that is 5 to 10 times smaller than an equivalent shell-andtube heat exchanger $[13,14]$. These characteristics appear to indicate that an MCHX could be suitable for a number of applications, including use in the Gen IV advanced reactor designs. M.G. Khan and A. Fartaj performed a review of the various applications, which include electronics and nuclear. They also surveyed many sources 
of literature and provided estimates of the percentage of literature sources that use various correlations for fluid flow and heat transfer within the heat exchanger or microchannels, such as friction factor, critical Reynolds number, or Nusselt number, but noted that accurate fluid flow correlations have not been established for microchannel flow yet [11]. Of specific importance is that the critical Reynolds number, $\operatorname{Re}_{\mathrm{cr}}$, is reported by many researchers to be lower for microchannels than for conventional tubes, and that transition flows in microchannels cannot be analyzed using traditional correlations before appropriate modifications are made. Finally, the lack of design data and experimental results for entire MCHXs is once again reiterated, and is certainly a motive for the work contained within this thesis. Despite this, a reasonable amount of information of the manufacturing methods utilized by companies that produce compact heat exchangers, such as Heatric Ltd., is available.

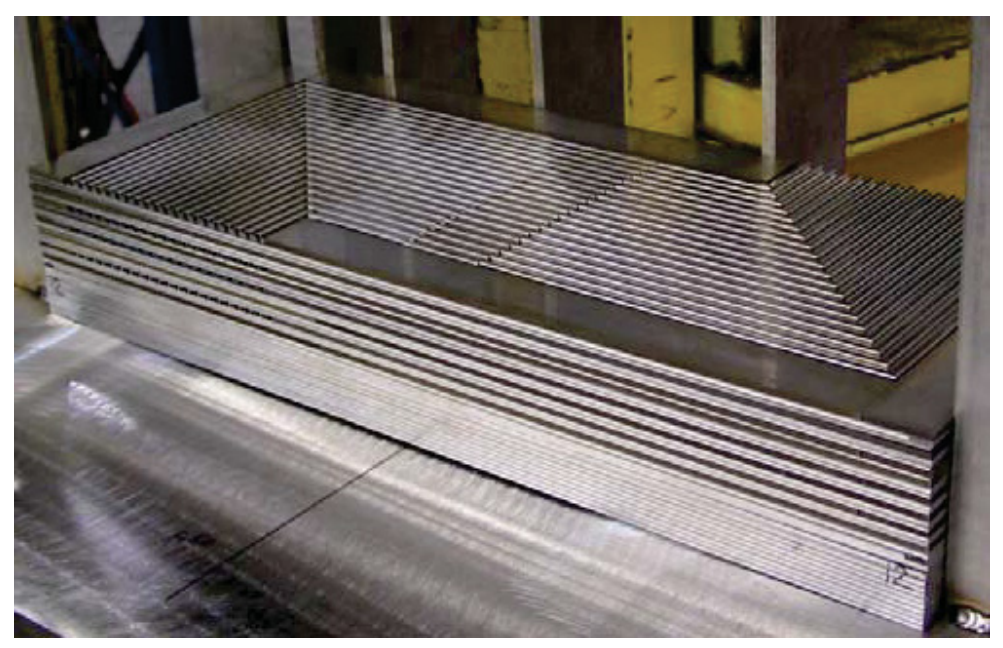

Figure 1.5: A set of chemically etched plates for an MCHX, stacked prior to diffusion bonding [9].

For example, D. Southall and S.J. Dewson provide an excellent overview of the manufacturing process of a Heatric MCHX in [9] and include a series of images detailing the process, such Figure 1.5 that shows the stacking of the chemically etched plates before diffusion bonding occurs. 


\subsubsection{Compact Heat Exchanger Analyses}

Although design data and experimental results for analysis of commercially available microchannel heat exchangers is lacking in the open literature, attempts have been made to create models and perform some analysis. As a start, M.M.A. Bhutta et al. have done a review of various computational fluid dynamics (CFD) methodologies used for the design and development of HXs, including an evaluation of some analyses performed by other authors on topics ranging from overall pressure drop and temperature differential to fouling and corrosion [15]. They note that the bulk of the analysis performed has been done using ANSYS CFX or Fluent. An attempt at performing a CFD analysis of a PCHE using ANSYS Fluent can be found in a thesis written by S.P Kar, where "the flow behaviour and heat transfer characteristics of semi elliptical and sinusoidal channels [were] studied successfully [16]." One of the larger conclusions drawn is that the performance of a particular channel geometry can be estimated when the ratio of the Colburn factor to the Fanning friction factor, $\mathrm{j} / \mathrm{f}$, is observed, where a value of 0.7 is deemed ideal. Further treatment of fluid flow and heat transfer in microchannels is available in [17], which provides a description of fluid dynamics in microchannels, and in [18], which contains information on two-phase flow within microchannels subject to nearly constant heat flux boundary conditions.

The potential for the MCHX technology to be used in the Gen IV advanced reactor designs has been noted by a number of authors as well. C.H. Oh et al. provide an introductory analysis of a PCHE for use in a very high temperature gas-cooled reactor concept design, with comparison to helical-coil and shell-and-tube HX types [19]. While the PCHE offered excellent thermal performance in a small and compact package, they estimated that difficulties regarding inspections and maintenance, as well as a lack of practical data for long-lifetime use, could be limiting factors in implementing 
the technology. S. Mylavarapu et al. examine PCHEs in a similar very high temperature reactor capacity, but provide an experimental perspective in addition to detailing the setup of the test facilities and fabrication of their own PCHEs [20]. K. Shirvan et al. perform an initial CFD analysis of a PCHE implementation into a compact medium size pressurized water reactor (PWR). The excellent thermal performance of the compact heat exchanger was noted once again, and their analysis showed that it could theoretically be possible to increase the reactor power by up to $50 \%$ when the technology is combined with an advanced fuel such as externally cooled annular fuel, while maintaining the same reactor vessel size. They also concluded that reactor design containing the PCHE was less expensive per unit electric power produced, and that while "there are many concerns in using PCHEs for long operating periods, its performance showed great potential to improve economics of any integral reactor design [21]."

There is also limited experimental research on the use of compact heat exchangers in a reactor-type capacity. J. Van Meter detailed the use of a PCHE in the Secure Transportable Autonomous Reactor developed by Argonne National Laboratories, a system that "allows for significant reductions in plant costs, size, and complexity, combined with a significant increase in plant efficiency through use of an advanced Brayton power conversion cycle using supercritical carbon dioxide," in an effort to evaluate the heat transfer characteristics of the exchanger when the working fluid is supercritical [22]. A methodology to estimate the internal geometry of a commercially available PCHE, provided by Heatric Ltd., was also developed, and both 2-dimensional and 3-dimensional CFD models were also created with ANSYS Fluent, which reproduced the supercritical carbon dioxide outlet temperatures to within nearly $15 \%$ and $3 \%$ respectively. In [23], K. Natesan et al. consider a PCHE for use in a specific application, being the intermediate heat exchanger in a very high 
temperature reactor design, which can be required to pass upwards of $50 \mathrm{MWt}$ to the intermediate loop in some configurations. The relevant thermal-hydraulic parameters and stresses are evaluated for the PCHE and shell-and-tube HXs in this particular application, and once again the compact HX is found to be superior. The allowable design life of the PCHE is estimated to be about 4 times greater than its shell-andtube counterpart, and they found that the "PCHE technology shows even greater heat exchanger volume reduction compared to the shell-and-tube configuration even with the surface fins [23]."

\subsubsection{Smoothed Particle Hydrodynamics}

When considering application of MCHXs within the Gen IV advanced reactor designs, it is necessary to account for the possibility of encountering multi-phase flow conditions. A typical fixed-mesh finite element method (FEM) is not well suited for the analysis of these types of problems, as it is extremely challenging to deal with the large volumetric changes between the liquid and the gas phases and the moving phase boundaries at the liquid-gas interface. Smoothed particle hydrodynamics (SPH), a Lagrangian formulation, has the potential to overcome this issue by tracking the properties of individual particles instead of observing mass and energy flux through fixed elemental volumes. D.J. Price provides an introduction to the method, "showing how the equations of motion and energy can be self-consistently derived from the density estimate [24]."

Use of SPH to model various fluid flow and heat transfer problems has already been successfully demonstrated. S. Nugent and H.A. Posch were able to apply the Lagrangian particle method to a liquid drop, surrounded by vapour, for "a Van der Waals (VdW) fluid exhibiting a liquid-gas phase transition below the critical point [25]." They were not only able to show stable drop configurations at various temperatures, 
but also generate oscillations in a drop, seen in Figure 1.6, that is initially deformed due to the VdW cohesive pressure. Successfully modelling surface tension is a highlight of that work.

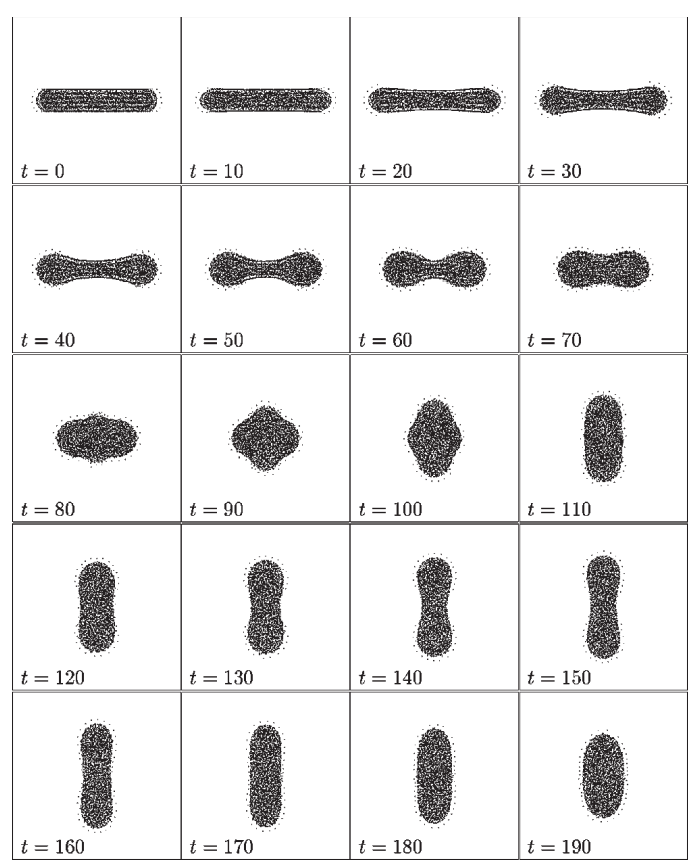

Figure 1.6: Observed oscillation in a VdW fluid drop, solved at various times using the SPH method [25].

SPH can also be used to model heat transfer problems. P.W. Cleary demonstrates the capabilities of the SPH method using test problems such as a discontinuity in the initial temperature within a small problem domain, heat conduction within a disk, and natural convection, all of which show excellent agreement with analytical solutions for each problem [26]. Cleary concludes that "SPH can accurately predict high temperature gradients with only small numbers of particles. The full transient evolutions are very accurate. Furthermore, any errors decay faster than the heat diffuses making the method extremely robust." He also notes that the "heat flux conservation across interfaces with different material properties... ensures accurate temperature profiles [26]." Finally, J.J. Monaghan et al. are able to demonstrate phase change via solidification using the SPH method. Using the Stefan problem as an example, 
they are able to produce results that accurately depict the transient evolution of both the number of solidified particles, as well the location of the solidification front and numerous instances in time [27]. The above cases demonstrate the power of the SPH method for modelling problems that would be very challenging using traditional fixed-mesh FEM techniques.

\subsubsection{Optimization}

Optimization of a design has historically occurred through attempts to cover as much of a design space as possible using exhaustive methods. However, it is possible to use statistical techniques to facilitate the process and catalyze the development of an optimal design in significantly fewer trials than the "brute force" methods. G. Box describes the benefits of using statistical methods, such as response surface methodology, and how the designer should not only use the results generated for a specific experiment to indicate which modifications to make, but to also iteratively update the design of experiments (DoE) to reflect any information garnered from previous trials $[28,29]$. The example in this case is the design of a paper helicopter. Box and Liu show that use of statistical methods allowed for a superior design with flight times nearly twice as long as their original prototype to be obtained in a much quicker fashion than if an exhaustive design methodology had been used. The importance of statistical methods in global optimization is further elaborated on by D.R. Jones in [30], where a handful of methods are outlined, including the aforementioned response surface methodology. The Kriging method, a basis-function method that has parameters that are tuned, is introduced here as well. Unlike other methods, use of the Kriging approach allows the designer to come up with an estimate of the potential error in the predictions of value for an objective function between design points. This can be exploited to selectively choose points within a design space that will minimize the overall uncertainty by the largest margin. What remains is a significantly stronger 
evaluation of an optimum solution [30].

As far as optimization of the MCHX design goes, there are varying stances on how to approach this problem. Typically, an economically-driven approach is used, where the optimal design is that which provided the lowest pressure drop, and thus lowest pumping requirement. H.J. Fontein and J.G. Wassink provide a brief overview of how to perform such an optimization on an arbitrary heat exchanger, basing their analysis on two kinds of costs: overall depreciation on investment and operating, or pumping, costs [31]. A.C. Caputo et al. perform a similar analysis using the total present cost, which is the sum of the capital investment cost and discounted operating cost, as the basis for their cost function. However, they also employ a genetic algorithm (GA), which starts "from an initial population of randomly created individuals representing the candidate solutions... [and] applies the principle of survival of the fittest to produce better performing individuals in subsequent evolutionary generations of the examined population [32]." In all examples, the solution produced by their GA was superior to the reference geometry pulled from literature for examination. A similar methodology is used by A. Hadidi and A. Nazari in their work on optimization of shell-and-tube heat exchangers using genetic algorithms, which produced similarly positive results of reduced operating and total costs for the optimized design [33]. Finally, A.G. Kanaris et al. performed an optimization on a plate heat exchanger with undulated surfaces using response surface methodology. While the use of statistical methods for optimization was recommended, a key point is that a balance should be struck between enhancement of heat transfer and reduction of frictional losses when creating a cost function for analysis of a HX [34]. Using this procedure, they were able to generate an optimal plate geometry, and were able to validate their CFD results using accepted values from literature. 


\subsection{Motivation and Outline of the Research}

As mentioned in the preamble of Chapter 1, it is desired to improve the overall performance of NPPs by not only using a more refined design than previous generations, but also by modifying and improving the components used. One technology that has the potential for use in the Gen IV reactor designs is the microchannel heat exchanger by virtue of its excellent heat transfer and mechanical properties, that are provided in a small package when compared to other similar technologies such as the much larger, more expensive, and maintenance-prone shell-and-tube HX. The aim of this work is to provide organization to the available MCHX research, primarily through an introduction of methods that can be used for analysis of the technology to aid in determining its suitability for the aforementioned application. With relatively little design information for commercial MCHXs available in open literature, development of a robust model and optimization thereof for use in nuclear reactors takes on significant importance [11]. It should be noted that this work is highly exploratory in nature where the models presented are mostly preliminary, and that the methods used to perform the analyses are the significant takeaway. Modelling of specific micro-scale effects or more complex effects associated with flows through microchannels, such as Ledinegg instability, have not been accounted for in these analyses and are beyond the scope of this work. These effects and experimental results have been discussed in open literature, and the reader is encouraged to explore works such as [35] or [36] for more detail.

\subsubsection{Thesis Outline}

The first analysis method, which is a traditional fixed-mesh finite element method, for the MCHX is introduced in Chapter 2. Beginning with the governing equations used, the VrSuite software that is developed by Goldak Technologies Inc ${ }^{\circledR}$ at Carleton 
University is presented. Brief mention of how operator splitting allows for coupling of the VrSuite solvers is also provided before detailing the FEM analysis performed on a sample MCHX design. The design presented is also used as reference design for the optimization performed in a later chapter.

To account for the possibility of multi-phase flows, an alternate solution method is proposed in Chapter 3. After an introduction to the smoothed particle hydrodynamics method, the details for the VrSuite SPH solvers will be presented in a similar manner to the information provided on the VrSuite FEM solvers in Chapter 2. Some small adaptations to the modelling method are outlined, which essentially manifest as changes in the primary variables used for specifying material properties from simply temperature to pressure, enthalpy, or both depending on the solver used. While most solver verification will be explicitly detailed in Appendix A, the various models created over the course of generating familiarity with the VrSuite SPH capabilities are presented in this chapter as they provide an outline of how the process of creating an SPH model of heated channel evolved, which should ultimately be used as the foundation for a future MCHX model. Additionally, it should be noted that interface thermodynamics have not been taken into account for problems involving phase change. A series of additional model adaptations that should be considered is proposed, closing the chapter.

With models created for the MCHX, the focus of this work turns towards performing an optimization of the design in Chapter 4, starting with a general overview of the concepts behind optimization, as well as a brief description of a handful of different optimization routines. This includes the Kriging optimization routine, which is ultimately chosen for the analysis performed. The general requirements to perform optimization are also outlined here. This is followed by the problem definition for the 
MCHX problem, an overview of VrSuite's optimization capabilities, and finally the results of the MCHX optimization.

A summary of the work performed and the major conclusions of this work are provided in Chapter 5. This includes the generation of an initial MCHX FEM model and corresponding results, a review of the additional modelling capabilities provided by the SPH methodology, and the optimizations performed for the MCHX technology. Some of the limitations of these models are also reviewed, and future work and considerations are also mentioned.

Please note that segments of this work have been adapted from two conference papers, which were written by this author as both the primary contributor and first author $[8$, $37]$. 


\section{Chapter 2}

\section{Microchannel Heat Exchanger Analysis with FEM}

To act as a starting point in the analysis and optimization of an MCHX, finite element methods offer a suitable balance between simplicity, depending on the level detail included in the models, as well as an opportunity to examine many different designs in a relatively short amount of time compared to more antiquated methods. Granted, FEM models can become extremely complex depending on the requirements for an analysis and the components which are included, but they provide a desirable level of flexibility and ease of implementation when used for preliminary analyses that do not require high levels of fidelity.

This chapter details the first steps of the MCHX analysis, including the initial FEM model that has been created of a single pair of channels in a HX. Furthermore, the initial model provides the added benefit of generating familiarity with the VrSuite software package, and is the basis for the model used in the expansion of the optimization problem presented in Chapter 4 to include FEM. To begin, an introduction to the VrSuite solvers, the governing equations used, and the data flow utilized for coupled thermal and fluid flow analyses are presented in the following sections. 


\subsection{VrSuite FEM Solvers and Governing Equa- tions}

VrSuite is a software package developed by Goldak Technologies Inc ${ }^{\complement}$, which is designed to solve coupled, transient problems in three spatial dimensions [38]. For solution of problems using FEM, it uses a number of different solvers that allow the user to evaluate individual phenomena, or a more complex system should the solvers be coupled. For the MCHX analysis, four solvers are used: the VrSuite Navier-Stokes (NS), advection-diffusion (AD), thermal, and stress solvers. Using them individually allows for fluid flow properties, such as velocity or pressure, or thermal properties, such as fluid temperatures, to be solved. Coupling them allows for a more complete analysis of the heat transfer that occurs from a flowing hot fluid, through the HX solid domain, and a flowing cold fluid, including stresses that are generated within the solid. More complete descriptions of these solvers follow.

\subsubsection{Governing Equations}

Many of the VrSuite FEM solvers use a traditional Eulerian approach, where conservation of mass, momentum, and energy occurs for fluxes through the fixed-mass elements that make up the meshed solution domain. The fluid flow solver obtains pressure and fluid velocity within the fluid domains using the incompressible continuity and momentum equations, Equations (2.1) and (2.2) respectively [39]:

$$
\begin{gathered}
\nabla \cdot \boldsymbol{v}=0 \\
\rho\left(\frac{\partial \boldsymbol{v}}{\partial t}+\boldsymbol{v} \cdot \nabla \boldsymbol{v}\right)=-\nabla P+\mu \nabla^{2} \boldsymbol{v}
\end{gathered}
$$

where $\mathbf{v}, \rho, \mathrm{t}, \mathrm{P}$, and $\mu$ are the velocity, time, density, pressure, and dynamic viscosity 
respectively. Ideally, a flow solver that uses the fully compressible continuity and momentum equations would be used, but was not available at the time of writing.

Solution of the temperature profile within the fluid domain can be achieved using the AD solver, which is governed by the following expression [40]:

$$
\frac{\partial T}{\partial t}=\nabla \cdot \alpha \nabla T-\boldsymbol{v} \cdot \nabla T
$$

where $\mathrm{T}$ is the fluid temperature and the thermal diffusivity is given by $\alpha=\mathrm{k} / \rho \mathrm{c}_{\mathrm{p}}$, with $\mathrm{k}$ representing the thermal conductivity and $c_{\mathrm{p}}$ being the specific heat capacity at constant pressure.

Determining the temperature profile within the solid domain entails use of the thermal solver. Conservation of energy, Equation (2.4) below, provides the means to do so [41]:

$$
c_{p} \frac{D T}{D t}-\nabla \cdot q^{\prime \prime}+Q=0
$$

where Q a volumetric heat generation term, and the heat flux is provided by Fourier's law in the form $\mathrm{q}^{\prime \prime}=-\mathrm{k} \nabla \mathrm{T}$.

Finally, the stresses within the solid domain can be computed using the VrSuite stress solver, governed by a combination of conservation of momentum with the inertial term $\rho(\partial \mathbf{v} / \partial \mathrm{t}+\mathbf{v} \cdot \nabla \mathbf{v})$ ignored, the stress-strain constitutive relation, and the relationship between displacement and strain, Equations (2.5), (2.6), and (2.7) respectively [40]:

$$
\nabla \cdot \sigma+b=0
$$




$$
\begin{gathered}
\sigma=E \varepsilon \\
\varepsilon=\frac{\nabla u+(\nabla u)^{T}+(\nabla u)^{T} \nabla u}{2}
\end{gathered}
$$

where $\sigma$ is the Cauchy stress tensor, $\mathrm{b}$ is a body force, $\mathrm{E}$ is the $6 \times 6$ elasticity tensor, $\varepsilon$ is the Green-Lagrange strain tensor, and $\mathrm{u}$ is displacement.

\subsubsection{Operator Splitting and Data Flow}

While it is possible to utilize the VrSuite FEM solvers individually to perform simplified analyses of the MCHX, such as determination of fluid flow properties within the channels using the NS solver or temperature fields within the solid domain using the thermal solver, a more complete examination of the technology that includes all the individual work flow elements should be evaluated. We wish to analyze a single pair of channels, where heat is transferred from the flowing hot fluid through the HX solid domain and into the cold fluid. While fairly involved, by taking advantage of symmetry it is possible to decompose this more complex problem into smaller, simpler sub-problems that can be treated individually. To accomplish this, the operators required for solution of a pressure and velocity field or temperature field can be split and subsequently utilized by the appropriate solver, and any data required to prescribe boundary conditions and state can be passed from one solver to another.

As mentioned in the preamble for Section 2.1, four VrSuite solvers are used for the MCHX analysis. The NS solver computes the fluid domain velocity and pressure fields by solving Equations (2.1) and (2.2), fluid domain temperature profiles are obtained via solution of Equation (2.3) by the $\mathrm{AD}$ solver, the temperature field in the solid

domain is solved by the thermal solver using Equation (2.4), and stresses within the 
solid domain are determined using Equations (2.5), (2.6), and (2.7) with the stress solver. To perform the desired analysis, the solvers are run in parallel and data is passed between them as described in the workflow diagram of Figure 2.1:

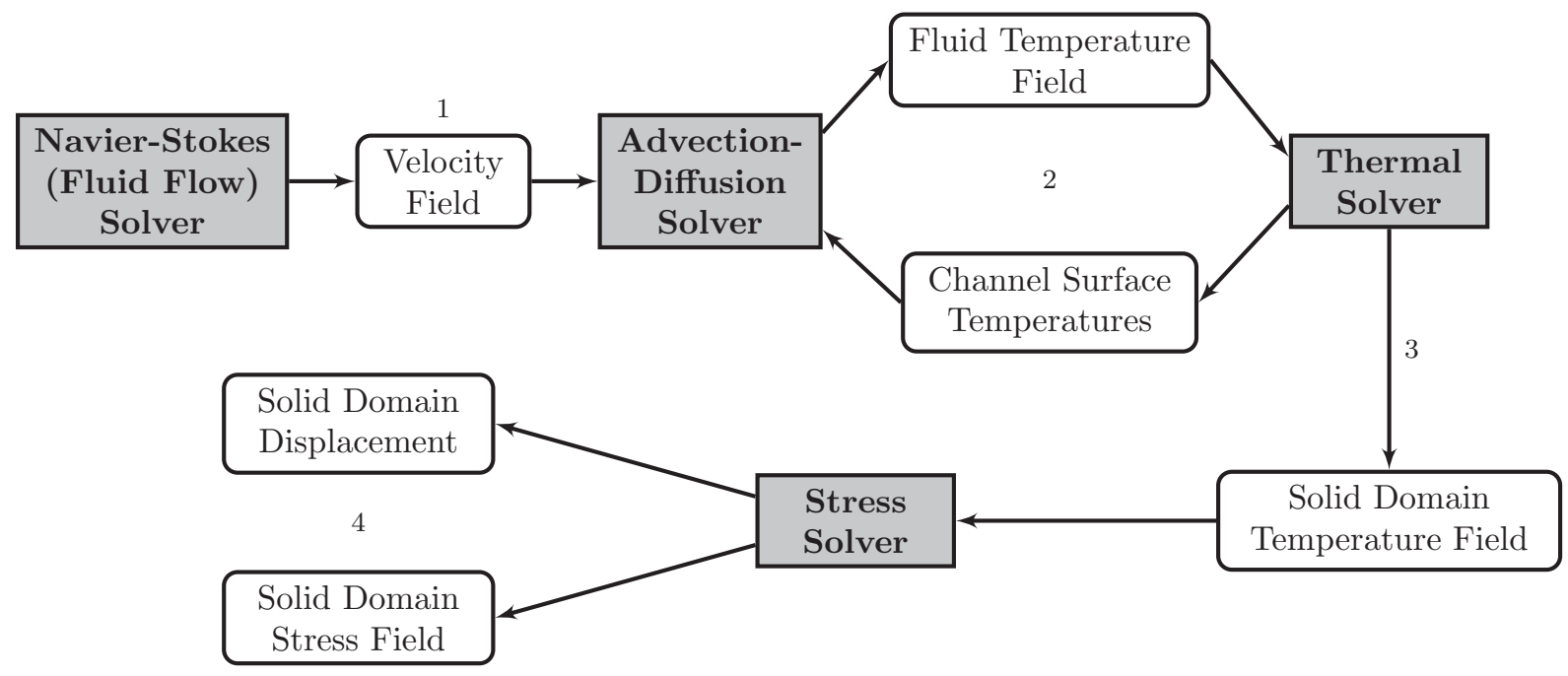

Figure 2.1: The data flow used by the VrSuite FEM solvers for coupled analyses.

The first step is to determine the velocity and pressure field within the fluid domain (1) using the NS solver, which is done independently. The solved data is then passed to the AD solver, which is coupled directly with the thermal solver. Beginning with prescribed boundary conditions (BCs) and initial conditions (ICs) for the fluid and solid domain temperatures, the AD solver computes the temperature at each location within both the hot and cold fluids. The temperature of each fluid at the surface of their respective channels is passed to the thermal solver to be used as a boundary condition for that time step (2). With that data, the thermal solver can determine the temperature profile within the entire solid domain, and passes the newly determined channel surface temperature to the AD solver. This process repeats for each time step, where the solid domain temperature at the end of the simulation is passed to the stress solver (3). The stress solver can then compute stresses and deformation within the solid domain (4). 
Notably, there is no interaction between the stress solver and the NS solver in the above procedure. To maintain simplicity for the initial MCHX analysis, it is assumed that any deformation caused by thermal expansion or hydrostatic stresses would be minimal and would not drastically change the velocity or pressure fields of either fluid, a claim which should be examined more thoroughly at a later time. The process described here can be adjusted to fully couple the solvers, starting once again with the NS solver and obtaining all other data before proceeding to the next iteration. At the end of each iteration, the stress and deformation data would be passed from the stress solver back to the NS solver to be used in computations for the subsequent iteration, and the process would repeat until the desired end time is reached.

\subsection{Heat Exchanger Analysis - Number of Trans- fer Units Method}

Heat exchanger analyses are typically classified into one of two types of problems: heat exchanger design problems and heat exchanger performance calculations. In the former, the designer will have knowledge of the inlet conditions and will attempt to determine a HX design that is capable of producing a specified outlet temperature. For the analyses in this work, the outlet temperatures remain unknown and must be obtained from either simulation or by calculation. This type of problem is classified as a HX performance calculation where a "heat exchanger [design] is analyzed to determine the heat transfer rate and the fluid outlet temperatures for prescribed heat flow rates and inlet temperatures [41]."

To perform the analysis, there are two commonly used methods: the log mean temperature difference (LMTD) method and the effectiveness-number of transfer units 
( $€$-NTU) method. Although the LMTD method is suitable for HX performance calculations, it becomes cumbersomely iterative when outlet conditions are not known, making the use of the $\epsilon$-NTU method preferable in such cases [41]. The following section provides a description of that method.

\subsubsection{The $\epsilon$-NTU Method}

For heat exchanger performance calculations, the $\epsilon$-NTU method provides a means to quickly evaluate the effectiveness of an HX by comparing the actual heat transfer rate to the maximum possible rate for the exchanger, $\mathrm{q}_{\max }$, that "could, in principle, be achieved in a [hypothetical] counterflow heat exchanger ... of infinite length. In such an exchanger, one of the fluids would experience the maximum possible temperature difference, $\mathrm{T}_{\mathrm{h}, \mathrm{i}}-\mathrm{T}_{\mathrm{c}, \mathrm{i}}[41]$." Use of this method begins by determining the heat capacity rate for both fluids, defined as $\mathrm{C}=\dot{\mathrm{m}} \mathrm{c}_{\mathrm{p}}$. Since the fluid undergoing the maximum possible temperature change in this hypothetical HX must have the smaller heat capacity rate, it is possible to calculate the maximum heat transfer rate:

$$
q_{\max }=C_{\min }\left(T_{h, i}-T_{c, i}\right)
$$

"where $\mathrm{C}_{\text {min }}$ is equal to $\mathrm{C}_{\mathrm{c}}$ or $\mathrm{C}_{\mathrm{h}}$, whichever is smaller [41]." A heat capacity ratio can also be defined using the heat capacity rates of both the hot and cold fluids as $\mathrm{C}_{\mathrm{r}}=\mathrm{C}_{\min } / \mathrm{C}_{\max }$, where $\mathrm{C}_{\max }$ is the larger rate. The effectiveness, $\epsilon$, of the heat exchanger can then be defined:

$$
\epsilon=\frac{q}{q_{\max }}
$$

Noting that the heat transfer rate can be expressed for either fluid as $\mathrm{q}=\mathrm{C}_{\mathrm{h}}\left(\mathrm{T}_{\mathrm{h}, \mathrm{i}}-\mathrm{T}_{\mathrm{h}, \mathrm{o}}\right)$ or $\mathrm{q}=\mathrm{C}_{\mathrm{c}}\left(\mathrm{T}_{\mathrm{c}, \mathrm{o}}-\mathrm{T}_{\mathrm{c}, \mathrm{i}}\right)$ for the hot or cold fluid respectively, it 
follows that the effectiveness can be defined alternatively as:

$$
\epsilon=\frac{C_{h}\left(T_{h, i}-T_{h, o}\right)}{C_{\min }\left(T_{h, i}-T_{c, i}\right)}=\frac{C_{c}\left(T_{c, o}-T_{c, i}\right)}{C_{\min }\left(T_{h, i}-T_{c, i}\right)}
$$

By definition, $\epsilon$ is a dimensionless quantity and must fall within the range $0 \leq \epsilon \leq 1$. Provided that the effectiveness is known for a particular HX, as well as the inlet conditions for both fluids, the method allows for easy computation of the actual heat transfer rates:

$$
q=\epsilon C_{\min }\left(T_{h, i}-T_{c, i}\right)
$$

A second quantity widely used for HX analysis is the number of transfer units, which can be defined using the overall heat transfer coefficient, $\mathrm{H}$, the heat transfer area, A, and the minimum heat capacity rate:

$$
N T U=\frac{H A}{C_{\min }}
$$

Knowledge of either $\epsilon$ or NTU allows for the other to be determined should the heat capacity ratio be known as well, and relations for these parameters are readily available in literature for various HX configurations. As an example, for the counterflow configuration that will be examined in this work, the following expressions apply [41]:

$$
\begin{aligned}
& \begin{cases}\epsilon=\frac{1-\exp \left[-N T U\left(1-C_{r}\right)\right]}{1-C_{r} \exp \left[-N T U\left(1-C_{r}\right)\right]} & C_{r}<1 \\
\epsilon=\frac{N T U}{1+N T U} & C_{r}=1\end{cases} \\
& \begin{cases}N T U=\frac{1}{C_{r}-1} \ln \left(\frac{\epsilon-1}{\epsilon C_{r}-1}\right) & C_{r}<1 \\
N T U=\frac{\epsilon}{1-\epsilon} & C_{r}=1\end{cases}
\end{aligned}
$$


Considering that $\epsilon$ is a function of NTU and $\mathrm{C}_{\mathrm{r}}$, which can both be determined from knowledge of the geometry and inlet conditions, the method is particularly powerful for this HX analysis and the initial optimization of an MCHX to be performed in Chapter 4 .

\subsection{MCHX FEM Modelling}

There are various approaches that can be used to create the initial models of an MCHX, which differ mostly by the level of complexity involved and depend on how much of the heat exchanger is modelled:

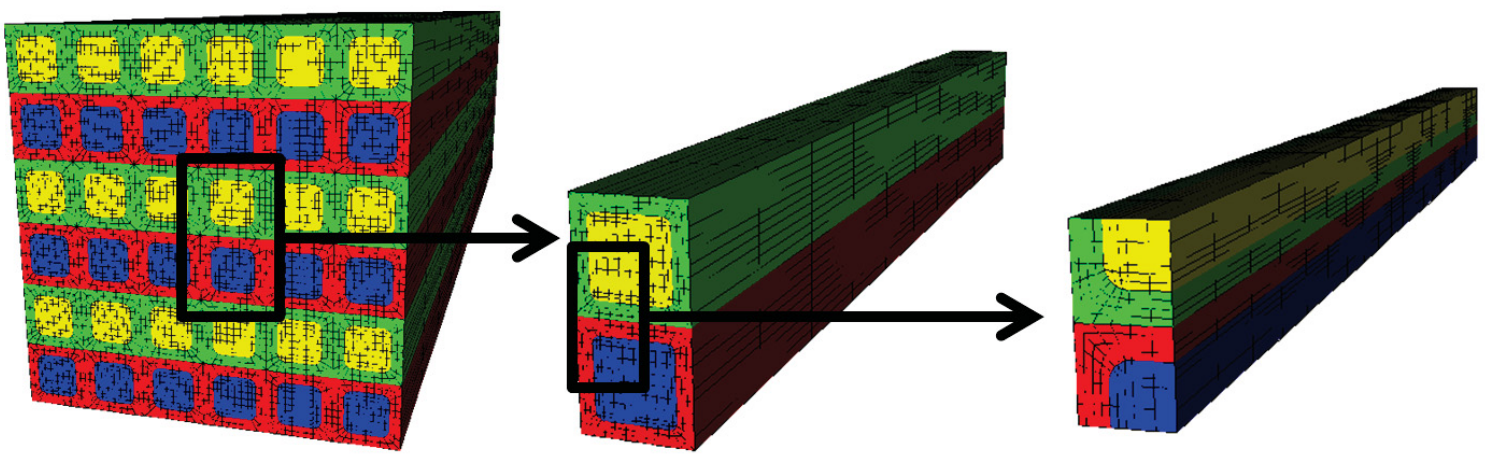

Figure 2.2: The three levels of complexity considered for the initial MCHX modelling attempts.

A "chunk" of an MCHX consisting of a series of plates each with multiple channels, seen at the far left of Figure 2.2, provides the greatest level of complexity of anything considered. While beneficial to capture heat transfer between channels of the same plate or from the HX to the surrounding environment, such a problem is more involved than is necessary for the initial analysis. A simpler problem should serve as a start for examination of an MCHX. Modelling a single pair of channels for a hot and a cold fluid stream, seen in the middle of the above figure, using simple single phase inlet conditions is a suitable option and is chosen for the initial analysis. The quarter channel pair model seen at the far right in Figure 2.2 will be discussed thereafter as 
an alternative to the full channel pair model, as it offers the possibility of a significant reduction in total computational load.

\subsubsection{Initial MCHX Model - Single Channel Pair}

The initial model created of an MCHX consists of a single pair of channels, one housing the hot fluid and the second for the cold fluid, set up in a counterflow configuration. This simplified numerical model was generated to create a functioning model from which familiarity with the software could be enhanced, and also to potentially be used as a basis model for optimization. Only the VrSuite NS, AD, and thermal solvers are used for the preliminary modelling efforts.

\section{Model Description}

The initial MCHX channel pair model uses two identical square channels with a width and height of $\mathrm{w}=2.75 \mathrm{~mm}$, and a length of $\mathrm{L}=50 \mathrm{~mm}$. A thickness between the channels of $\mathrm{L}_{\text {wall }}=2.75 \mathrm{~mm}$ is also used. The geometry is selected somewhat arbitrarily given the preliminary nature of the model, although the channel size is intentionally chosen to remain very close to the "micro" scale dimensions of currently available MCHXs.

The solution domain for this problem is split into three parts, composed of an FEM mesh with 1680 8-node volume elements: the MCHX solid domain, the hot fluid domain, and the cold fluid domain. The mesh generated by the VrSuite MCHX mesh type can be seen in Figure 2.3. The hot and cold fluid domains are represented by the yellow and blue components respectively while the MCHX solid is composed of both the red and green elements. Note that although two distinct meshes are used to create the solid domain, this is simply a result of the VrSuite meshing process and the solid domain is continuous without any interfaces, that is, the mesh is conforming. 


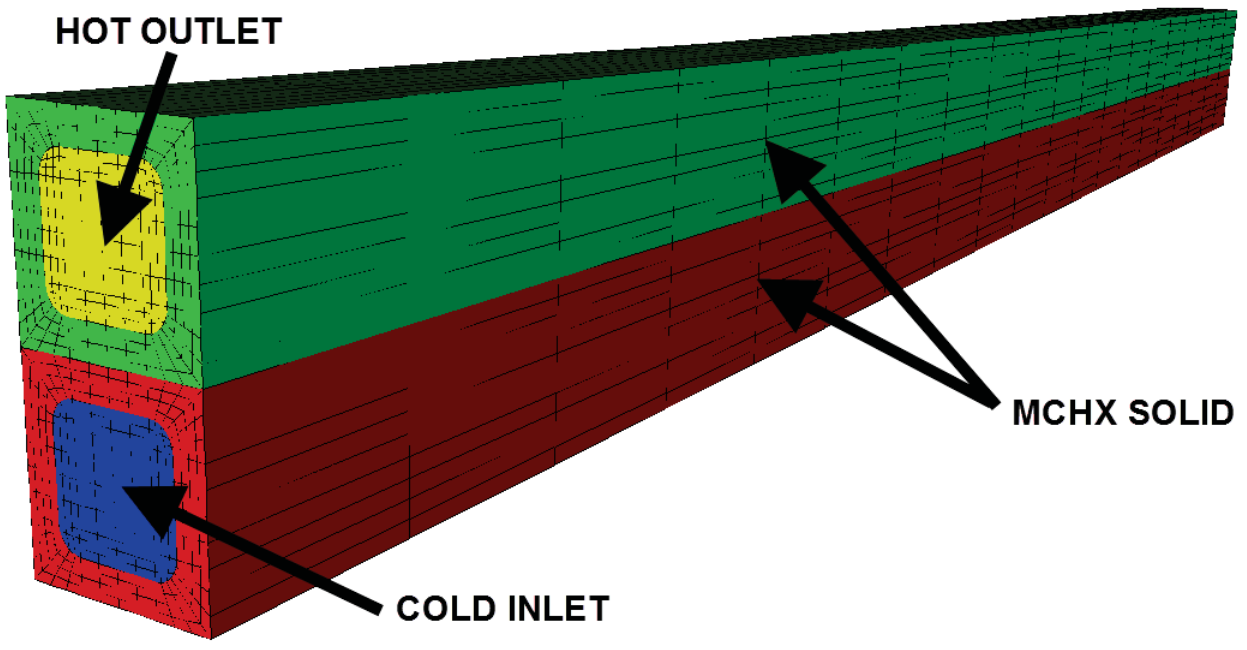

Figure 2.3: The mesh used for the full channel pair model.

The inlet conditions are selected arbitrarily for the hot and cold fluid, being set to $\mathrm{T}_{\mathrm{c}}=25^{\circ} \mathrm{C}, \mathrm{T}_{\mathrm{h}}=75{ }^{\circ} \mathrm{C}$, and $\mathrm{P}_{\mathrm{c}, \mathrm{h}}=1$ atm to maintain simplicity for the initial test by ensuring single phase flows within both channels. The inlet normal velocity of both fluids is set to be $\mathrm{v}_{\mathrm{c}, \mathrm{h}}=1 \mathrm{~m} / \mathrm{s}$ for similar reasoning. Additionally, there is no back pressure applied to the outlet of either channel. Symmetry BCs are applied to the outer surfaces of the HX model to represent the HX that would exist around the channel pair analyzed.

For HXs used in nuclear applications, INCONEL alloy 600 or 690 are commonly used materials, having thermal conductivities of roughly $\mathrm{k}=15.0 \mathrm{~W} / \mathrm{m} \cdot \mathrm{K}$ and $\mathrm{k}=12.1 \mathrm{~W} / \mathrm{m} \cdot \mathrm{K}$ respectively for the temperature ranges chosen for the analysis $[42,43]$. The average of the two, $\mathrm{k}_{\mathrm{wall}}=13.55 \mathrm{~W} / \mathrm{m} \cdot \mathrm{K}$, will be used for this initial modelling effort. Water is used for both fluids, and the fluid properties are set to match those available from the National Institute of Standards and Technology (NIST) for the given operating conditions [44]. 


\section{Results}

When the initial channel pair model is allowed to reach a steady state for the given inlet conditions, occurring around $t=6 \mathrm{hrs}$, the following temperature fields and corresponding fluid temperature profiles are obtained. Note that only half of the solid domain is shown to provide a clearer visualization of the inside surface of both channels:
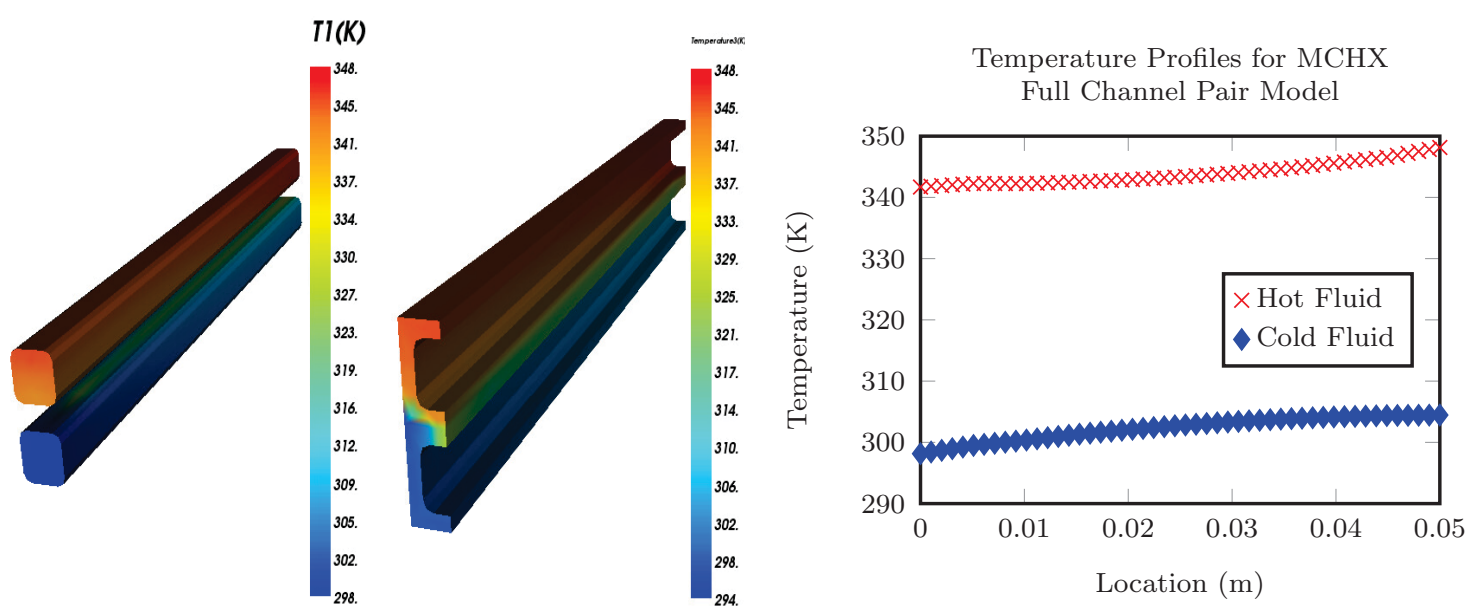

Figure 2.4: Fluid (left) and solid (mid) domain temperature fields, with both fluid temperature profiles (right) for the full channel pair model.

Visualizations of the temperature fields for the fluid and solid domains, seen in the left and middle images of Figure 2.4 respectively, appear to indicate that heat is being transferred from the hot fluid into the MCHX solid and through to the cold fluid as intended. The extreme high and low temperatures exist where the BCs are specified, near the top-right for the hot fluid inlet and at the bottom left for the cold fluid inlet in both domains. While the general trends are representative of a typical temperature profile for a heat exchanger in the counter-flow configuration, there exist slightly steeper temperature gradients near the fluid inlets at the locations $\mathrm{x}=0 \mathrm{~m}$ and $\mathrm{x}=0.05 \mathrm{~m}$ for the hot and cold fluid respectively. A possible explanation is the lack of an inlet plenum for either the hot or cold fluid in this simplified MCHX model, 
likely causing entrance effects and flow development near the fluid inlets to not be captured entirely. Alternatively, the coarse mesh used here could account for this feature of the results.

The simulated outlet temperatures for the cold and hot fluids of $\mathrm{T}_{\mathrm{c}, \mathrm{o}, \mathrm{sim}}=31.31{ }^{\circ} \mathrm{C}$ and $\mathrm{T}_{\mathrm{h}, \mathrm{o}, \mathrm{sim}}=68.54{ }^{\circ} \mathrm{C}$ also correspond well to the $\mathrm{T}_{\mathrm{c}, \mathrm{o}, \text { ana }}=31.50^{\circ} \mathrm{C}$ and $\mathrm{T}_{\mathrm{h}, \mathrm{o}, \text { ana }}=68.37^{\circ} \mathrm{C}$ determined using the $\epsilon$-NTU method, yielding total temperature changes in either fluid that differ by no more than $2.92 \%$. Examination of the obtained heat transfer rates and HX effectiveness' shows a similarly reasonable comparison, with a relative difference of $2.30 \%$ between $\mathrm{q}_{\text {sim }}=200.3 \mathrm{~W}$ and $\mathrm{q}_{\text {ana }}=205.0 \mathrm{~W}$, and a relative difference of $3.01 \%$ between $\epsilon_{\text {sim }}=0.129$ and $\epsilon_{\text {ana }}=0.133$. These results and the excellent agreement with their analytical counterparts are summarized in Figure 2.5, where each of the simulated values are normalized relative to their respective analytical values and a value of 1 indicates a perfect match.

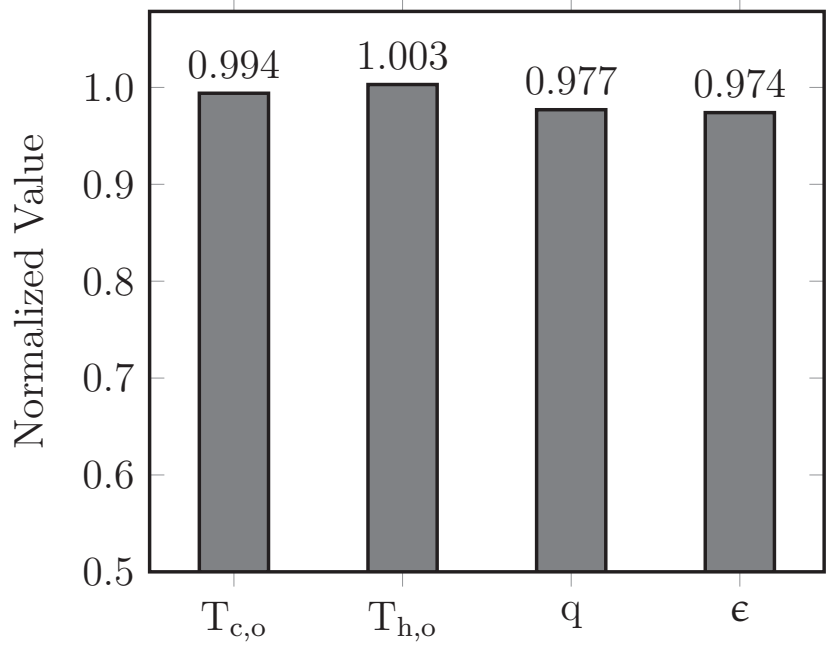

Figure 2.5: Full channel pair FEM results normalized relative to their respective analytical values.

Notably, the effectiveness values produced are not as high as the ideal value mentioned to be possible for MCHXs in Chapter 1, likely due in part to the limitations of this 
model. Foremost, only a single pair of channels are being modelled and use of a more detailed model, such as one that includes an array of channels described in the preamble for Section 2.3, could produce more physically realistic results. Inclusion of inlet plenums for both fluids would also likely smooth the gradients seen in the inlet regions, and provide a more accurate representation of any entrance effects that would occur. Furthermore, the channel sizes chosen for this model are very close to "micro" scale dimensions. Additional consideration should be given to proper modelling of the physics of liquid flows in microchannels, whose behaviour is not terribly well understood at this point and is beyond the scope of this work [45].

Despite this, the preliminary model presented here still serves to demonstrate how FEM may be used as a tool for future modelling efforts for the MCHX technology. The agreement between the simulated results and those produced by use of the $\epsilon$-NTU method suggests that the model is representing an MCHX with a level of plausibility that is acceptable for an initial model, and provides a good sanity check for model functionality and proper solver coupling. These results also suggest that this model could be used as the basis for the initial optimization of an MCHX using FEM that is presented in Chapter 4.

\subsubsection{Mesh Sensitivity Analysis}

While the results obtained using the presented FEM model of an MCHX appear to agree very well with the solution provided using the $\epsilon-\mathrm{NTU}$ method, it is prudent to perform some measure of mesh sensitivity analysis. Using the same geometry and BCs as the initial MCHX FEM model, successive increases to the total element count were made, using the simulated heat transfer rates and effectiveness' as the parameters upon which mesh sensitivity was evaluated. The mesh refinements and their simulated values are summarized in Table 2.1, using bold for the initial model. 
Table 2.1: Summary of models used for a mesh sensitivity analysis and the corresponding results.

\begin{tabular}{|c|c||c|c||c|c|}
\hline $\begin{array}{c}\text { Total } \\
\text { Element } \\
\text { Count }\end{array}$ & $\begin{array}{c}\text { Physical } \\
\text { Solve Time, } \\
\mathbf{t}_{\text {solve }}[\mathrm{hrs}]\end{array}$ & $\begin{array}{c}\text { Heat Transfer } \\
\text { Rate, } \\
\mathbf{q}_{\text {sim }}[\mathbf{W}]\end{array}$ & $\begin{array}{c}\text { \% Difference } \\
\text { From } \\
\text { Previous }\end{array}$ & $\begin{array}{c}\text { Exchanger } \\
\text { Effectiveness, } \\
\epsilon_{\text {sim }}\end{array}$ & $\begin{array}{c}\text { \% Difference } \\
\text { From } \\
\text { Previous }\end{array}$ \\
\hline \hline $\mathbf{1 6 8 0}$ & $\mathbf{1 . 8 1}$ & $\mathbf{2 0 0 . 3}$ & N/A & $\mathbf{0 . 1 2 9 2}$ & N/A \\
\hline 4320 & 3.49 & 203.6 & 1.63 & 0.1282 & 0.79 \\
\hline 6400 & 13.4 & 204.1 & 0.23 & 0.1285 & 0.23 \\
\hline 9600 & 15.7 & 202.2 & 0.93 & 0.1273 & 0.93 \\
\hline 15600 & 35.3 & 202.7 & 0.27 & 0.1276 & 0.27 \\
\hline
\end{tabular}

The trends that exist for the values shown in Table 2.1 are readily visible when presented graphically:
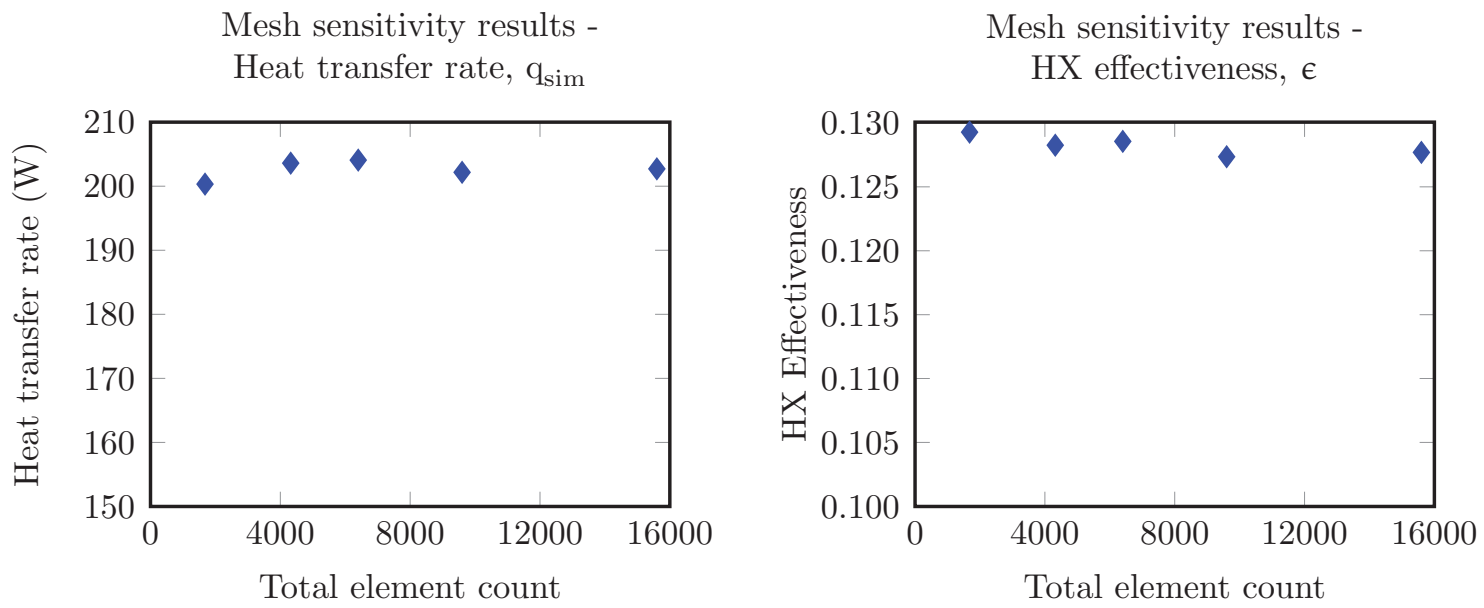

Figure 2.6: Variation in heat transfer rate (left) and exchanger effectiveness (right) with changes to total element count.

Both of the plots presented in Figure 2.6 show that for each variable of interest, very little change occurs despite a significant increase in the total element count from the 1680 elements used for the initial model. Between any two subsequent models there is no more than $1.63 \%$ change in the value for either $\mathrm{q}_{\text {sim }}$ and $\epsilon$, which indicates that 
the mesh utilized for the initial model is likely adequate for both this preliminary analysis and the optimization to be performed. A small increase in the overall heat transfer rate can also be noted near a total element count of approximately 4000, suggesting that the initial model is also slightly conservative. Examination of the physical solve time for each model further supports the use of the initial mesh. Not only do the results for the initial model correspond well with those produced using the $\epsilon-N T U$ method, the fully-transient, three-dimensional, coupled analysis is performed in a reasonably short $1.81 \mathrm{hrs}$ of total computation time. The required solve time increases quite drastically when the total element count is raised, without providing a solution that is either significantly different or more accurate than that generated with the initial model.

\subsubsection{Model Refinement - Quarter Channel Pair}

While the channel pair model discussed in Section 2.3.1 produced acceptable preliminary results and built familiarity with the VrSuite software effectively, there is room to introduce some modelling refinements going forward. Specifically, modelling only a quarter of the channel pair and applying symmetry BCs to the planes where the "cuts" are made allows for the mesh to be refined without incurring significant extra computational load, or to directly reduce the computational overhead without making adjustments to the mesh. These boundary conditions serve to replace the remainder of the MCHX channel pair that is not modelled.

The refined model would be mostly identical to the full channel pair model detailed above, except only a quarter of the initial model would be examined. The channel dimensions and applied BCs would remain the same, in addition to the newly applied symmetry boundary conditions. All inlet conditions for both fluids, as well as the material properties for the fluids and MCHX solid would also remain unchanged. 


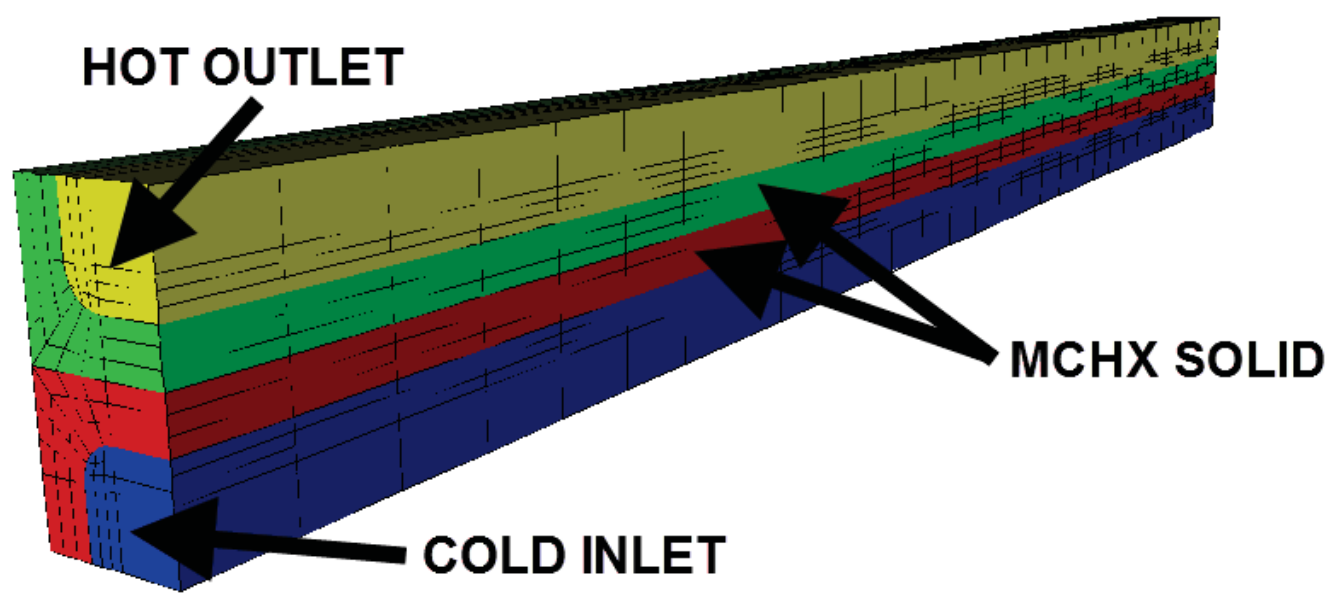

Figure 2.7: The mesh proposed for the refined quarter channel pair model.

From the mesh used for initial model, the suggested refinements would produce a solution domain mesh for the refined model identical to that shown in Figure 2.7. The yellow and blue components represent the hot and cold fluid domains respectively, and the red and green elements compose the MCHX solid.

Given that the computational time required for the initial model is quite reasonable, introduction of mesh refinements would be the focus. For example, the density of elements near the channel walls could be increased in order to better capture boundary effects. Referring once more to the mesh presented in Figure 2.7, a total of 2400 8node volume elements would be used, which is an approximately a $42.9 \%$ increase in total element count despite the model only being a quarter of the total volume. The total required solve time would be expected to be comparable to the $3.49 \mathrm{hrs}$ of the 4320 element model examined in the mesh sensitivity study shown in Section 2.3.2. However, given that the initial model has been deemed adequate for demonstrating the application of FEM in a preliminary analysis of an MCHX and as a basis for the optimization to be performed in Chapter 4, performing the analysis using the quarter channel pair model is suggested as a future endeavour. 


\section{Chapter 3}

\section{Introduction to Smoothed Particle Hydrodynamics}

Having developed a working model of an MCHX channel pair and selected a reference design for optimization, the focus of this work shifts towards an analysis method that can more easily capture complicated phenomena, such as flows with sharp discontinuities in material properties or moving phase boundaries, that are difficult to treat with traditional fixed-mesh FEM. Smoothed particle hydrodynamics is a novel method that provides a Lagrangian framework to solve such problems with relative ease compared to FEM, and is introduced in the following section.

\subsection{What is Smoothed Particle Hydrodynamics?}

$\mathrm{SPH}$ is a mesh-independent numerical technique that can simulate complicated flows by using 'fluid' particles to represent a flow field. One such application is multi-phase flows with interacting fluids, where the movement of the particles is tracked relative to each other to represent a denser fluid, which is usually water, and a lighter fluid, which is usually steam. While mass, momentum, and energy are conserved with both FEM and SPH, a Lagrangian reference frame is used in the latter to provide the framework 
for the method as opposed to the Eulerian reference frame that is sometimes used in FEM. Where mass and heat fluxes are typically evaluated for volume elements in Eulerian methods, use of a Lagrangian reference frame allows for modelling of a domain by using a series of individual particles whose "properties (e.g. temperature) [change] in accordance with a set of differential equations derived from the original governing PDEs," or partial differential equations [26]. All properties of interest, such as enthalpy or mass, are tracked on a per-particle basis, and momentum and heat fluxes are determined by considering a region of interest around each individual particle when the SPH method is employed. A sample SPH particle discretization as compared to a typical FEM mesh can be observed in Figure 3.1.

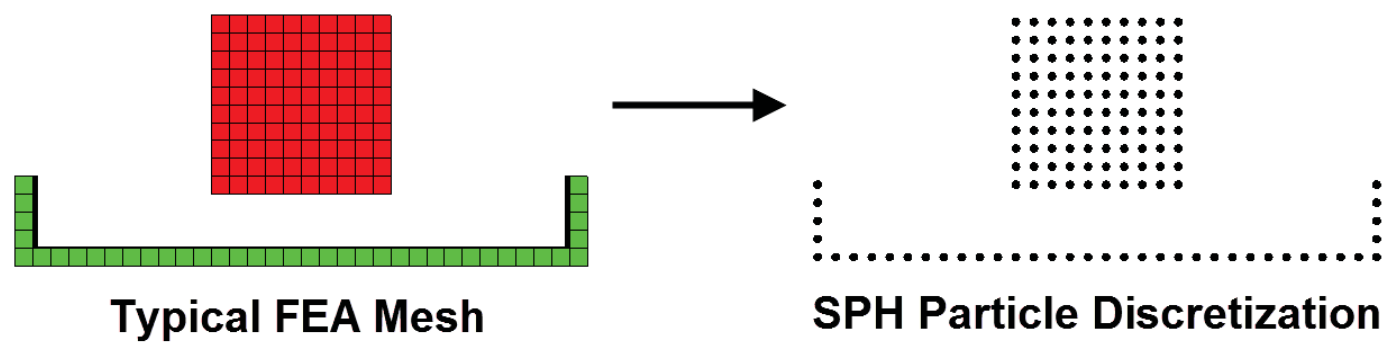

Figure 3.1: A typical FEM mesh (left) and comparable SPH discretization (right).

This method provides a number of benefits, ranging from the relatively easy treatment of momentum-dominated flows, to modelling interactions between different substances, to solution of problems with complicated "physics such as multi-phase, realistic equations of state, compressibility, radiation, solidification and fracturing," and extension to three-dimensions [26]. SPH provides the means to solve the problem of tracking an interface or complex free surfaces, which is extremely difficult to solve using FEM. The following sections detail the key SPH models created in support of the current MCHX modelling efforts, which include developing familiarity with and verifying the functionality of the SPH solvers implemented into the VrSuite software package, beginning with an outline of the equations that drive the solvers. 


\subsection{VrSuite SPH Solvers and Governing Equations}

\subsubsection{Governing Equations}

With a traditional FEM approach, conservation of mass, momentum, and energy are applied for fluxes through the individual volume elements that comprise the solution domain. When employing SPH, modified forms of the continuity, momentum, and energy equations, Equations (3.1), (3.2), and (3.3) respectively, are used [26]:

$$
\begin{gathered}
\frac{d \rho_{a}}{d t}=\sum_{b} m_{b}\left(v_{a}-v_{b}\right) \nabla W_{a b} \\
\frac{d v_{a}}{d t}=-\sum_{b} m_{b}\left[\left(\frac{P_{b}}{\rho_{b}^{2}}+\frac{P_{a}}{\rho_{a}^{2}}\right)-\frac{\xi}{\rho_{a} \rho_{b}} \frac{4 \mu_{a} \mu_{b}}{\left(\mu_{a}+\mu_{b}\right)} \frac{v_{a b} \cdot r_{a b}}{r_{a b}^{2}+\phi^{2}}\right] \nabla_{a} W_{a b}+g \\
\frac{d U_{a}}{d t}=\sum_{b} \frac{4 m_{b}}{\rho_{a} \rho_{b}} \frac{k_{a} k_{b}}{k_{a}+k_{b}} T_{a b} \frac{r_{a b} \cdot \nabla_{a} W_{a b}}{r_{a b}^{2}+\phi^{2}}
\end{gathered}
$$

Here $\mathrm{m}, \mathrm{v}, \mathrm{P}, \mu, \mathrm{r}, \mathrm{U}, \mathrm{k}$, and $\mathrm{T}$ are the mass, velocity, pressure, dynamic viscosity, position, internal energy, thermal conductivity, and temperature of a particle. The subscripts a and b denote variable values for arbitrary particles a and b, with subscript ab denoting the value of a variable for particle a minus that for particle $b$. Using the method, valuation of a function for a particle a at some position $\mathrm{r}$ occurs by summing the values of all particles $b$ within a radius that is double the specified interpolation length $\mathrm{X}$. For example, the density of particles $\rho$ in a region is determined by interpolating between the masses of the particles in the specified surrounding area of influence. $\mathrm{W}$ is a spline-based interpolation function with a radius of $2 \mathrm{X}$ that "mimics the shape of a [Gaussian distribution] function but without the 
infinite tails," which governs the magnitude of the contributions made by the surrounding particles b on the particle a of interest for each evaluated expression [26]. Lastly, $\xi$ is a constant found in the modified viscous term of the momentum equation holding a value of $\xi=4.96333$, which has been determined by calibration against an exact solution for a form of Couette flow [26]. The modified terms used in these formulations ensure that stress and heat flux are continuous across material interfaces, which allow for multiple materials with densities, viscosities, thermal conductivities, and specific heats that are different by up to three orders of magnitude to be accurately simulated. In addition to these equations, it is also necessary to employ an equation of state to determine pressure, closing the system of equations for the SPH discretization [46]:

$$
P=B\left[\left(\frac{\rho}{\rho_{0}}\right)^{\gamma}-1\right]
$$

where $\gamma$ typically has a value between 1 and 7 , with 7 being chosen for this application [26]. Here, "B is the constant related to the bulk modulus of elasticity of the fluid" given by $\mathrm{B}=\mathrm{s}_{0}^{2} \rho_{0} / \gamma$, with $\mathrm{s}_{0}$ representing the speed of sound at the reference density $\rho_{0}[46]$. Please note that verification of the VrSuite SPH solvers utilizing these equations can be found in Section A.2 of Appendix A.

\subsubsection{Operator Splitting and Solver Data Flow}

As was done for the coupled FEM models presented in Chapter 2, the operators required for solution of a pressure and velocity field or a temperature field can be split and subsequently utilized by different solvers. Comparable to what was detailed in Section 2.1.2, this allows for a decomposition of a more complex problem into smaller, simpler sub-problems that can be treated individually. The SPH analyses performed here utilize two solvers: the VrSuite SPH Navier-Stokes (SPH-NS) solver 
and the VrSuite SPH thermal solver. The former provides pressure and velocity using the continuity and momentum equations, (3.1) and (3.2) above, and the latter solves the energy equation, (3.3), for enthalpy and temperature. While possible to solve free-surface flows or heat transfer problems using either of the solvers individually, operator splitting allows for data to flow from one solver to the other. This data flow effectively couples the two and provides a means to model more complex phenomena, such as boiling.

For coupled analyses, such as those detailed in subsequent sections, the data flow will typically begin with the SPH-NS solver. Once the initial conditions are specified for the set of particles comprising the domain, the SPH-NS solver will obtain new velocities for each of the particles and update the pressure field for the next timestep. This data is then passed to the SPH thermal solver, which uses the updated particle positions when solving the energy equation. A new temperature distribution is obtained, and is passed back to the SPH-NS solver for use in calculations for the next time step. This process iterates until the specified end time is reached.

\subsection{Required Data Pre-Processing}

Although it is possible to run either of the VrSuite SPH solvers by specifying material properties that are functions of a single variable, such as temperature or pressure, some pre-processing may be required should a problem involve multi-phase flows. For solution of such problems, the currently implemented SPH solvers would need to be coupled to fully capture the phase change phenomena, making it is necessary to use material properties that are functions of pressure and enthalpy. This also requires a change to how BCs and ICs are specified by the user, where all temperature values would instead by given by the enthalpy that corresponds to the desired temperature 
at the given initial operating pressure.

Water is the fluid used for the majority of this research, for which data is readily available online via the National Institute of Standards and Technology website [44]. However, the data available there is in terms of pressure and temperature, necessitating pre-processing. A piece of MATLAB code has been written to perform the required conversion from a function of pressure and temperature to a function of pressure and enthalpy, and is functional for any substance that has thermodynamic tables of material properties.

\subsubsection{Material Property Solver}

Given a range of pressures and enthalpies desired by the user, the code provides the properties of interest by performing a series of linear interpolations between pressure and temperature values of the existing data. A sample of the dynamic display and resulting output can be seen in Figure 3.2 below:

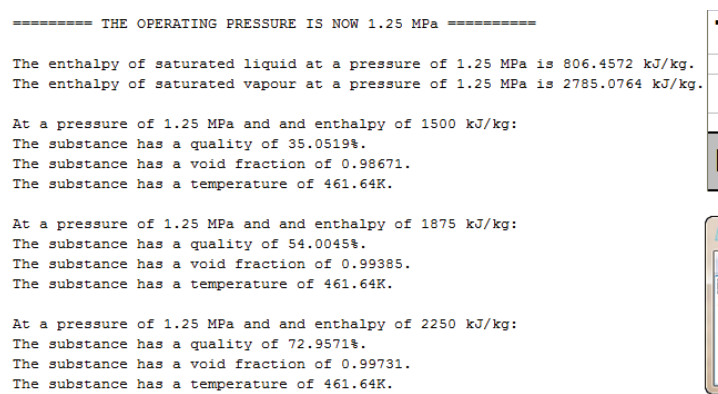

Figure 3.2: Code dynamic display (left) and both output file types (right).

As the property solver runs, the user is able to monitor the state of the substance at a given pressure and enthalpy, including temperature or quality and void fraction if applicable. Two outputs are generated when the run completes: a Microsoft Excel file used to facilitate inspection of the resulting data, and a three line text file which contains the desired data in a format supported by the VrSuite materials library. The 
first two lines contain enthalpy and pressure data while the third is the property data corresponding to each possible pair of the primary variables. Solution of two-phase flows using the SPH solvers becomes possible once each property text file is copied into the VrSuite materials library as two-argument tables.

\subsection{Developed SPH Models}

This section outlines the models that have been created using the recently implemented VrSuite SPH solver capabilities, with a focus on developing familiarity with and verifying the functionality of the SPH solvers in an effort to create a working MCHX model. As mentioned, the VrSuite SPH Navier-Stokes and SPH thermal solvers will be used for the analyses presented here. It should be emphasized that for problems where phase change occurs, interface thermodynamics are not taken into account. Additionally, note that fluid material properties are specified using either pressure or enthalpy alone as a primary variable for the NS and thermal solvers respectively, or both for models using coupled NS and thermal solvers. Section headers will indicate the solvers used for each model: SPH Navier-Stokes (NS), SPH thermal, or coupled NS and thermal solvers.

\subsubsection{Free Surface Flow: Falling Drop (NS)}

To begin developing familiarity with the SPH solvers, a simple model of a free surface flow was created utilizing only the SPH Navier-Stokes solver. A fluid 'drop' is created using a collection of 2500 particles, arranged as a 50 row by 50 column square with an initial particle spacing of $2 \mathrm{~mm}$. This arrangement of particles was chosen arbitrarily, with the coarse particle spacing selected to reduce computational load for this test. The basin is created with a series of particles spaced identically to the fluid drop, with a width of $300 \mathrm{~mm}$ and $30 \mathrm{~mm}$ tall walls, and remains fixed in space throughout 
the simulation. The drop is initialized at an arbitrary height of $40 \mathrm{~mm}$ above a basin, and allowed to free-fall once the simulation begins.

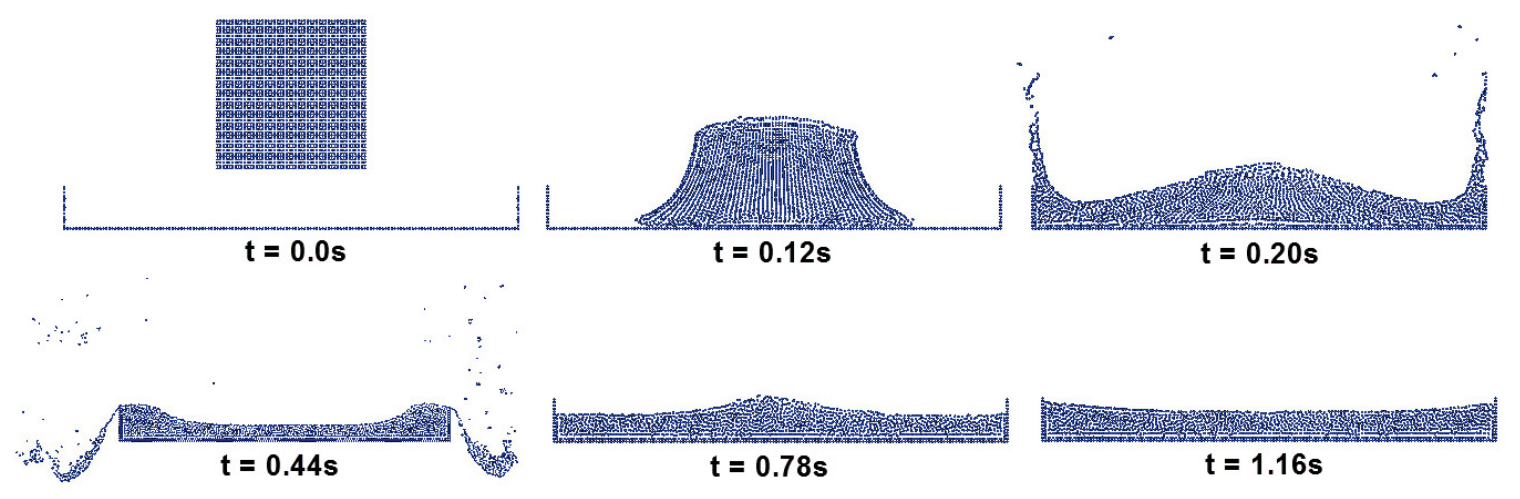

Figure 3.3: Temporal evolution of particle location for a free-falling liquid 'drop'.

The resulting particle formations at different instances in time are depicted in Figure 3.3, with the initial state shown at the top left of the image. After contact is made with the basin, the fluid drop starts to deform. The particles that were located near the bottom of the drop are pushed outwards towards the side walls of the basin (top right). The momentum carried by the particles causes them to jump upwards once contact with the side walls occurs, where they inevitably fall past the sides of the basin (bottom left). At this point, there is an accumulation of particles within the basin at the side walls, which generates a wave that propagates back towards the centre. The two fronts collide in the middle to create a new accumulation (bottom middle), which finally disperses back towards the edge of the basin (bottom right). The location of the particle build-up continues to oscillate as time progresses further, which is an expected result. Although the analysis is primarily qualitative, this example demonstrates the ease of modelling free surface flows using SPH, which would otherwise be a challenging problem if traditional fixed-mesh FEM techniques were used. These results also instil confidence that the SPH-NS solver is functioning as intended given the plausible particle motion that also appears to agree with independently performed simulations, such as in [47]. 


\subsubsection{Free Surface Flow: Inlet and Outlet (NS)}

The VrSuite SPH functionality also allows for the introduction of particle inlets and outlets, which is of particular interest for the creation of a functioning MCHX model. Another free surface flow model using the SPH-NS solver was created using an inlet and an outlet, where particles are introduced into an empty basin that is fixed in space, similar to the falling drop model presented in Section 3.4.1. Particles are injected into the domain through a $10 \mathrm{~mm}$ tall inlet located at the left-hand side with a velocity of $0.1 \mathrm{~m} / \mathrm{s}$, and are allowed to fall into a basin that is $40 \mathrm{~mm}$ wide and 10 $\mathrm{mm}$ deep after traversing over a flat wall that is $10 \mathrm{~mm}$ long. Once again, a particle spacing of $2 \mathrm{~mm}$ is used for all components of the model. A $10 \mathrm{~mm}$ tall zero pressure outlet is located at the top half of the right-hand side of the basin.

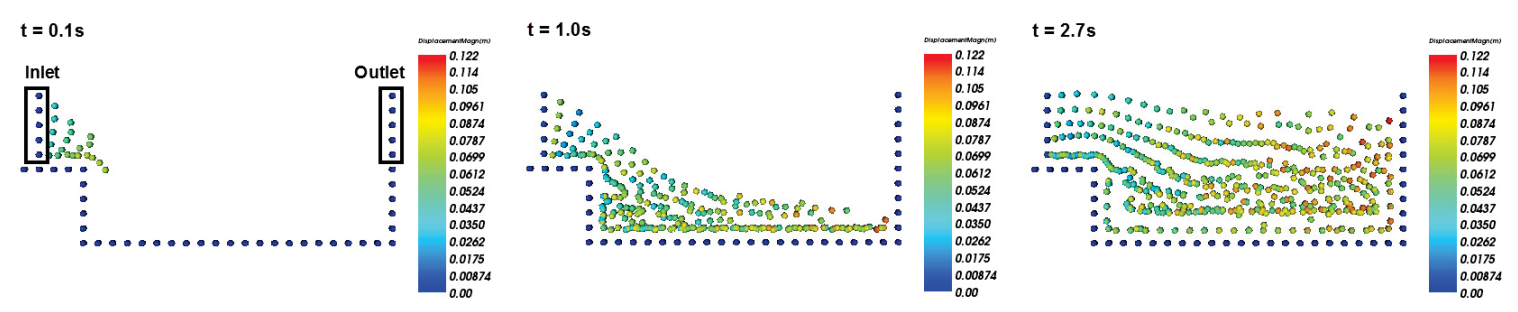

Figure 3.4: Free surface flow with an inlet on the left-hand side and an outlet on the top half of the right-hand side.

Initially, there are no particles present within the basin portion of the model. As particles are injected through the inlet, the fluid level within the basin steadily rises until the free surface reaches the bottom of the outlet and particles are able to leave the domain, which occurs at a rate that is sufficiently quick to prevent the particles from overflowing. While not apparent from the images seen in Figure 3.4, an animation of the flow reveals the presence of some back flow and possibly eddy formation in the corner near the backwards-facing step, another expected result. Unfortunately the resolution of these flow features is somewhat poor in this example, which is a problem that can be rectified should a greater number of particles with a tighter spacing be 
used. Although this analysis of the VrSuite SPH-NS solver is preliminary, observation of the expected flow patterns builds confidence in the functionality of the solver. It is recommended that a more thorough assessment of the solver functionality be performed, including a finer particle distribution that could more accurately capture the flow phenomena observed here.

\subsubsection{Fluid Solidification: The Stefan Problem (Thermal)}

Having examined the utility of the VrSuite SPH-NS solver, focus shifts towards use of the SPH thermal solver for evaluation of problems involving heat transfer. Specifically the SPH method can be applied to problems involving phase change, which can occur within the MCHX channels during operation. Modelling the Stefan problem is a suitable choice for demonstrating this capability. The basis for this problem is the solidification of a fluid, where the entire domain is initially liquid with a Dirichlet temperature BC applied to one side that is significantly lower than the melting temperature of the fluid. As time progresses, the domain will progressively change phase from liquid to solid, and the location of the solidification front will shift accordingly. When the SPH method is used, heat is removed from liquid particles by those that have been previously solidified, starting at the interface between the fluid domain and where the low-temperature $\mathrm{BC}$ is applied, eventually prompting a change in phase from liquid to solid.

\section{Model Description and Analytical Solution}

For this problem, the solution domain is constructed using a stationary 50 particle by 10 particle array, with a spacing of $20 \mathrm{~mm}$. The top and bottom surfaces of the domain are adiabatic, and all BCs, ICs, and physical parameters are normalized and chosen to match the values provided in [27] for comparative purposes. Here, the density $\rho=1$, specific heat capacity at constant pressure $c_{p}=1$, thermal conductivity 
$\mathrm{k}=1$, melting temperature $\mathrm{T}_{\mathrm{m}}=1$, and a property-dependant constant $\lambda=0.5$ are used. The Dirichlet temperature $\mathrm{BC} \mathrm{T}_{1}=0.1906$ is applied at the location $\mathrm{x}=0$ over the entirety of the simulation. The location of the solidification front must be determined before the temperature profile, which is dependant on the particle phase, and can be analytically determined as follows [27]:

$$
x_{S F}=2 \lambda \sqrt{\frac{k t}{\rho c_{p}}}
$$

Particles located at $\mathrm{x}<\mathrm{x}_{\mathrm{SF}}$ will be solid and those located at $\mathrm{x} \geq \mathrm{x}_{\mathrm{SF}}$ are still fluid. The temperature profile throughout the domain for this problem can now be determined analytically [27]:

$$
\begin{cases}T(x)=T_{1}+\frac{\left(T_{m}-T_{1}\right)}{\operatorname{erf}(\lambda)} \operatorname{erf}\left(x \sqrt{\frac{\rho c_{p}}{4 k t}}\right) & x<x_{S F} \\ T(x)=T_{0}+\frac{\left(T_{m}-T_{0}\right)}{\operatorname{erfc}(\lambda)} \operatorname{erfc}\left(x \sqrt{\frac{\rho c_{p}}{4 k t}}\right) & x \geq x_{S F}\end{cases}
$$

\section{Results}

The visualized domain temperature and solid fraction (left), as well as the temperature profile within the solution domain (right), are visualized in Figure 3.5 at $\mathrm{t}=0.058$, which was the value chosen for the analysis performed in [27]. Note that a solid fraction of $\mathrm{FS}=1.00$ indicates a particle has undergone a phase change from liquid to solid.

Examining the temperature profile, the two independently performed VrSuite SPH analyses using the same particle discretization match the analytical solution very well, where all simulated data falls within $5.66 \%$ of the corresponding analytical value. Additionally, the simulated location of the solidification front can be determined by observing the location where the temperature profile 'kinks'. The simulated 


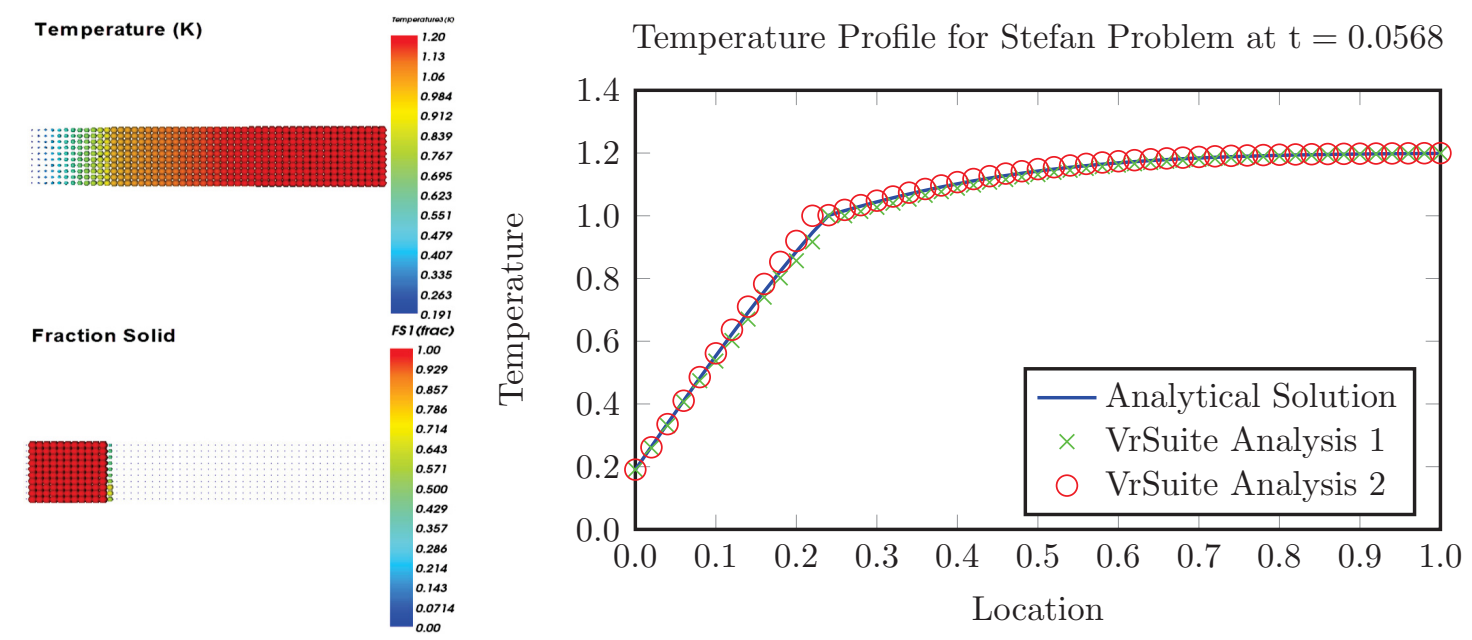

Figure 3.5: Visualized temperature and fraction solid (left) and corresponding solved temperature profile (right) for the Stefan problem at $\mathrm{t}=0.0568$.

location of $\mathrm{x}_{\mathrm{sim}} \approx 0.225$ compares very well to the analytically determined solution of $\mathrm{x}_{\mathrm{ana}}=0.2383$, differing by only $5.6 \%$. The excellent agreement of the simulated results with the analytical solutions indicate that the SPH can be used to model phase change, and that the VrSuite SPH thermal solver is functioning in this capacity. The disparities in the results observed here are most likely due to minute differences in the solver parameters specified for the two independently performed simulations and the coarse SPH particle density. This test has also been extended to model phase change between liquid and vapour particles, producing similarly agreeable results that will not be shown here.

\subsubsection{Pool Boiling of Water (Coupled NS and Thermal)}

Having successfully modelled free surface flow and heat transfer problems individually, an attempt was made to model flow boiling by coupling the two VrSuite SPH solvers using the method outlined in Section 3.2.2. As described in Section 3.3, both pressure and enthalpy are used as primary variables to specify material properties, BCs, and ICs, resulting in temperatures being represented by pressure-enthalpy pairs. This 
first model is of pool boiling, where a container of water is heated by applying a Dirichlet temperature boundary condition that is significantly higher than the boiling temperature of the liquid.

The data set used to specify the material properties for water, which is used by all components of the model, was limited to a small range. Pressures vary from $150 \mathrm{kPa}$ to $250 \mathrm{kPa}$ and enthalpies range from $100 \mathrm{~kJ} / \mathrm{kg}$ to $3100 \mathrm{~kJ} / \mathrm{kg}$, which corresponds to a maximum temperature range of approximately $282 \mathrm{~K}$ to $587 \mathrm{~K}$. The solution domain is comprised of two parts: a stationary container and the fluid particles that constitute the pool. The container is $50 \mathrm{~mm}$ wide and $60 \mathrm{~mm}$ tall, and the fluid domain is initially discretized as a 25 row by 25 column square array of particles. While an initial particle spacing of $2 \mathrm{~mm}$ is used for all components, the fluid particles settle into a more compact arrangement after the first time step that occupies roughly a third of the initial volume. Heating of the fluid occurs by ramping up the temperature at the bottom of the container steadily, but is specified as a Dirichlet enthalpy boundary condition given the requirement of enthalpy as a primary variable. Initially a BC of $\mathrm{h}_{1}=100 \mathrm{~kJ} / \mathrm{kg}$ corresponding to an initial Dirichlet temperature of $\mathrm{T}_{1} \approx 283 \mathrm{~K}$ is applied, and is ramped at a rate of $150 \mathrm{~kJ} / \mathrm{kg} / \mathrm{s}$ to a maximum of $\mathrm{h}=3100 \mathrm{~kJ} / \mathrm{kg}$, which corresponds to $\mathrm{T} \approx 586 \mathrm{~K}$.

The first indication of bubble formation in Figure 3.6 occurs around $t=20.0 \mathrm{~s}$, with a uniform layer of bubble-like structures forming along the bottom surface, evidenced by a distinct difference in volume surrounding the particles relative to the closer-packed liquid particles elsewhere in the container. The image for $\mathrm{t}=40.0 \mathrm{~s}$ shows the growth of the initial bubbles until just before buoyant forces are great enough to detach them from the surface of the pot. By $\mathrm{t}=64.1 \mathrm{~s}$ it is possible to see a fully detached bubble near the left side of the vessel, in addition to a few other bubbles that have previously 


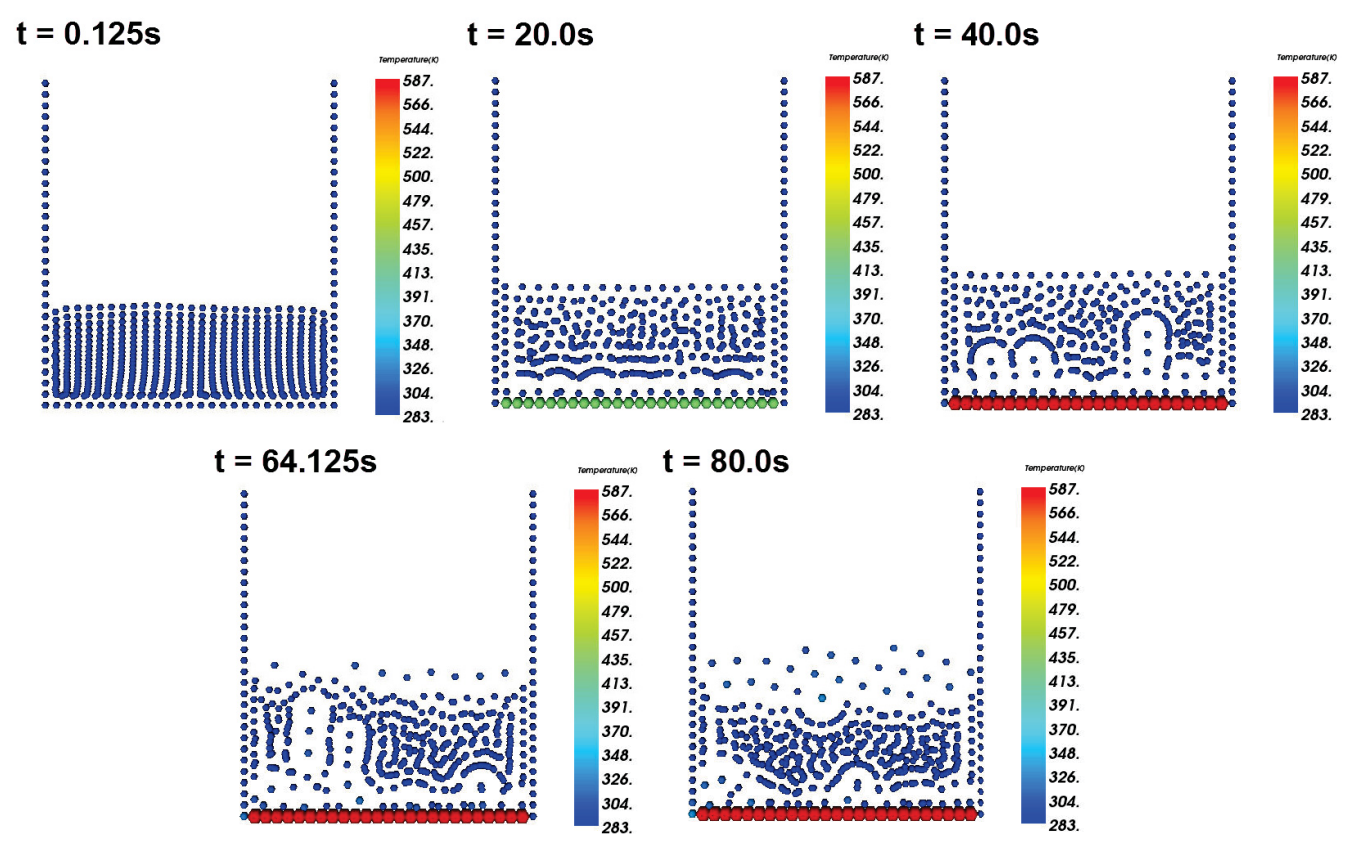

Figure 3.6: Development and growth of vapour bubbles along the bottom surface of a liquid pool as time elapses.

detached and burst, indicated by the collection of vapour particles accumulated along the free surface. Finally, the remaining image on the bottom right of Figure 3.6 shows continued bubble formation, along with a drastic decrease in the water level when compared to the initial state. Successful creation of an initial boiling model that appears to qualitatively simulated the observed physics is a significant result, and instils confidence that resolving boiling within an MCHX channel is possible. As was the case with the free surface and inlet/outlet flow models presented earlier in this section these results are only preliminary, and while mostly qualitative are still effective in demonstrating the capabilities of the SPH method to model complex fluid phenomena.

\subsubsection{Heated Channel Flow (Coupled NS and Thermal)}

Expanding upon the previous successes in demonstrating both phase change and boiling, a heated channel model serves as the foundation for generating a multichannel SPH model of an MCHX. Incorporating an inlet and outlet similar to what 
was presented in Section 3.4.2, a $40 \mathrm{~mm}$ long channel is created using two walls consisting of 200 particles with a spacing of $0.2 \mathrm{~mm}$. A $2 \mathrm{~mm}$ tall inlet injects liquid particles into the initially void channel with a constant normal velocity of $2.25 \mathrm{~m} / \mathrm{s}$, which are able to exit the channel at the opposite end through a zero-pressure outlet. As was done for the pool boiling model in Section 3.4.4, the VrSuite SPH-NS and SPH thermal solvers are coupled and water with the same material properties is used. An identical ramping Dirichlet BC to that seen in the previous section is applied to both walls of the channel.

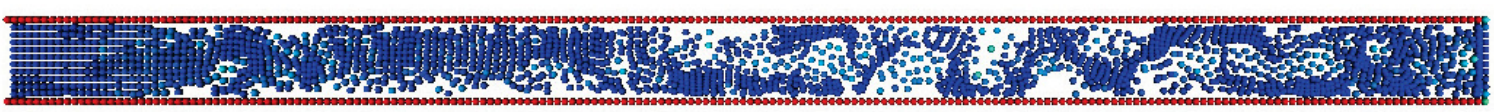

Figure 3.7: Boiling flow in a heated channel with inlet at the left-hand side and zero-pressure outlet at the right-hand side.

In Figure 3.7 the phase of the water particles clearly changes from liquid to superheated vapour during transit through the channel, evidenced by the numerous collections of bubble-like structures that can be identified by a difference in volume around the light blue particles of higher temperature compared to the dark blue lower temperature particles entering the channel. An animation of the flow provides a visualization of the two-phase flow regime, which evolves as the mass quality increases with continual application of heat. In a horizontal channel, the initial boiling will typically produce a flow containing many small bubble features that will transition to plug flow and eventually stratified flow, which are recognized through larger bulletshaped bubbles and distinct strata of liquid or vapour respectively [41]. Some of these flow regimes appear to be replicated by the heated channel model, where the first particles that experience phase change from liquid to vapour produce very small 
bubbles that are dispersed throughout the fluid media, and subsequent heating appears to yield larger bubble structures and a two-phase flow that mimics plug flow. It is expected that continued application of heat would produce stratified flow and subsequently annular flow, which is distinguishable by a core of high-velocity vapour surrounded by an annulus of fluid [41]. While these results are only preliminary and qualitative, they adequately demonstrate the capabilities of SPH to resolve boiling flows, and offer confidence in the method and solvers.

\subsection{Possible Model Adaptations}

While it is encouraging to see qualitatively plausible results for problems involving complex flow phenomena such as boiling, these models are still preliminary and offer room for improvement. The number of particles and particle density used in most of the models is coarse, which was done in order to improve computational speed. Improved resolution of the flow features demonstrated could be achieved by increasing the number of particles used and reducing the initial spacing. The analyses presented in this chapter have also been predominantly qualitative for problems involving the SPH-NS solver. A more complete examination of those results is prudent, but also requires additional support. Currently it is not possible to extract some flow data, such as a velocity profile for a user specified location, which would be required for a proper treatment of the simulated results. As another option, expansion of the models presented in this chapter to three dimensions is also recommended. While the solvers are using fully three-dimensional SPH equations, the models have been created to be only a single layer 'thick' to reduce the complexity of these problems to twodimensional analyses. Bridging the gap from a single layer to three-dimensions only requires changing a single global scalar constant, creation of the three-dimensional SPH particle discretization, and re-application of the BCs. 


\section{Chapter 4}

\section{Optimization of the MCHX Design}

While the use of the VrSuite FEM and SPH solvers have been demonstrated to be sufficient for analysis of a particular MCHX design, outlining a method to determine an optimal design for application in the Gen IV reactors is the focus of this work. The analyses detailed in the preceding chapters provide the foundation for performing an optimization of the technology, and are the first methods proposed for examining a compact heat exchanger. A method that can be used for optimization of an MCHX design is provided in this chapter, with a focus on the capabilities of the VrSuite software to aid in this endeavour.

Although it is desired to use the more robust SPH models of the MCHX as a basis for an initial optimization of the technology to be able to account for two-phase phenomena, the VrSuite optimization tool does not currently support SPH modeling. Additionally, the optimization tool cannot handle problems requiring coupling of the VrSuite FEM solvers. As such, flow conditions are specified to ensure single-phase fluids, and the optimization problems presented in this chapter either use analytical solutions or models created using traditional fixed-mesh FEM requiring only a single solver. 


\subsection{Optimization Methods}

Computational power and speed are steadily increasing, and engineering analyses that are being performed for a consistently growing number of design problems continue to increase in complexity, be it through the code used or the desired level of fidelity. "In the early stages of design, the focus is on generating, evaluating, and comparing potential conceptual configurations," and the designer will usually attempt to measure the impact of varying design parameter values [48]. It is often impractical to use complex, full FEM or CFD for these early analyses. As an alternative, metamodelling techniques can be employed, where metamodels act "as 'surrogates' [for] the expensive simulation process," and can be an effective tool to aid in "design optimization, including model approximation, design space exploration, problem formulation, and solving various types of optimization problems [49]."

Development of a metamodel typically consists of choosing a method for the design of an experiment such as brute force exhaustive design or Box-Behnken, picking a model to represent the data, and then fitting the model to observed data [48]. While there is a multitude of metamodelling techniques, a few of the more prevalent in current literature are response surface methodology, neural networks, inductive learning, and Kriging. Each method has distinct advantages. In brief, response surfaces are well suited to applications with random error, neural networks use a non-linear regression approach that can easily treat deterministic problems, and inductive learning is most useful "where the input and output factors are primarily discrete-valued or can be grouped into categories [48]."

Kriging is a global optimization method used to minimize the uncertainty in a design space, originally "developed in the field of spatial statistics and geostatistics," and 
applied for mining exploration models [50,51]. It is designed for use with deterministic data, and offers a number of advantages over the other methods, including the ability to "provide an exact interpolation of the data or "smooth the data'," as well as compensate for the effects of data clustering by giving less weight to points located within a cluster than individual points [48]. Additionally, Kriging models are generally more accurate for nonlinear problems, but at the expense of added complexity. Of great interest is the ability of the method to provide estimates of error and uncertainty in the value of an objective function. This allows for the algorithm to selectively choose locations within a design space to perform evaluations of a specified objective function in subsequent iterations of an optimization routine to most efficiently search for optima. These benefits form the basis for use of the Kriging method for the initial optimization of the MCHX technology seen here. For a more in depth treatment of these optimization methods, including a detailed overview of the Kriging method, the reader is encouraged to see the works of T.W. Simpson, G.G. Wang, and S. Jeong $[48-50]$.

\subsubsection{Local and Global Optimization}

Before outlining the requirements for solving an optimization problem, a distinction should be made between local and global optimization. A global optimization pertains to searching for minima or maxima for an entire design space or set of values, whereas local optimization only provides minima or maxima within a constrained subset of the original design space or data set. Although there is some localization of points in regions of the design space that have the greatest uncertainty in the objective function value for each iteration, Kriging is a global optimization algorithm given that the design points chosen can be located anywhere within the domain. 


\subsubsection{General Requirements for Performing Optimization}

Prior to performing an optimization, the designer must first define a design space, an objective function, and any constraints that apply to the analysis. For the design space, each of $\mathrm{N}$ design variables are constrained by an upper and lower bound, and an initial set of $\mathrm{M}$ test points within these bounds are selected to create a base experimental set. Typically, this involves the creation of an $\mathrm{M} \times \mathrm{N}$ design of experiment, or DoE matrix, where each of the $\mathrm{M}$ rows represents an experimental analysis, and each of the columns contains design values for one of the $\mathrm{N}$ design variables of interest. An objective function must also be specified, whose value is obtained for each analysis and serves as the key design metric for evaluating the fitness of each experimental design. After specifying any applicable design constraints, an optimization routine such as Kriging can be run to obtain a design that corresponds to an optimum value for the objective function. This usually involves minimizing or maximizing the objective function, although it is possible to also specify a target value.

\subsection{VrSuite Optimization Capabilities}

The VrSuite software package offers design optimization capabilities, including use of the Kriging method. When using the method, the software begins a run by selecting a set of sampling points using any acceptable statistical method, such as fractional factorial or Latin hypercube. For each Kriging iteration, the code defines an additional set of sample points based on its algorithm. Note that while experimental data has historically been used, simulations are used in this work for prediction of the behaviour for any design within the specified design space. A simple example of these capabilities is demonstrated here using the classical problem of one-dimensional heat conduction into a semi-infinite solid. Of the three BCs typically chosen for this problem (Dirichlet, Neumann, or Robin), a Neumann BC is selected here. 


\section{Model Description and Analytical Solution}

The VrSuite FEM thermal solver is utilized for this test case. The solution domain consists of a $1 \mathrm{~m}$ long steel bar with square $10 \mathrm{~mm}$ by $10 \mathrm{~mm}$ ends, discretized as 200 equalized sized 8-node volume elements as shown in Figure 4.1:

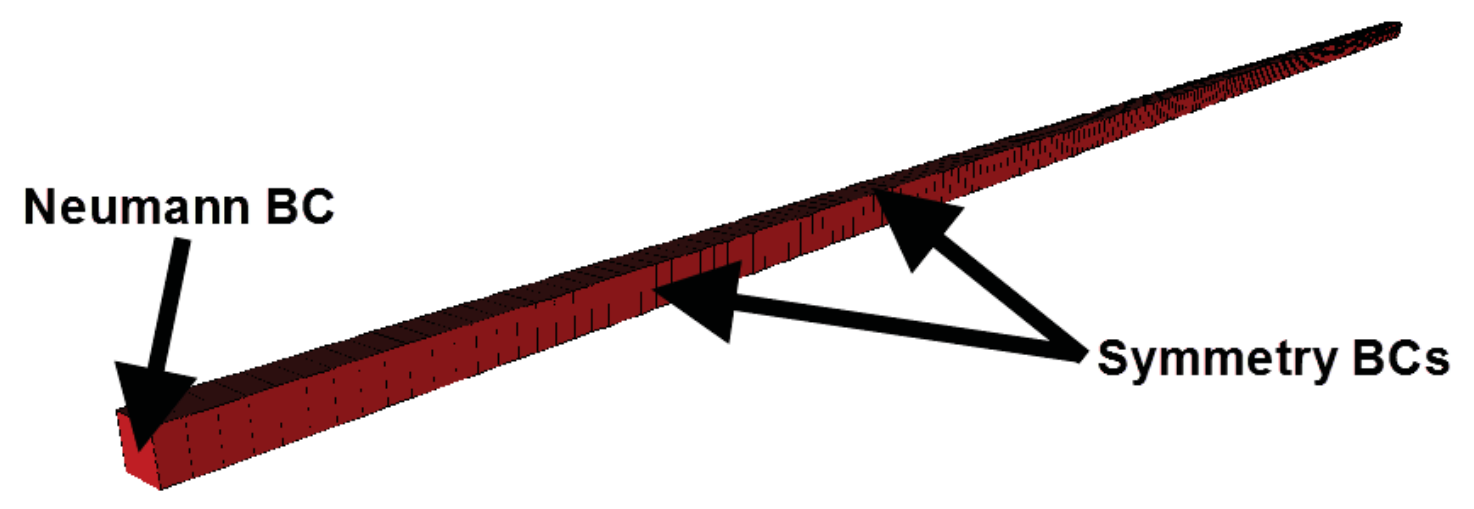

Figure 4.1: The mesh and applied BCs used for demonstration of the VrSuite optimization capabilities (heat conduction into a semi-infinite solid).

A Neumann BC of $-\mathrm{k} \partial \mathrm{T} /\left.\partial \mathrm{x}\right|_{\mathrm{x}=0}=100 \mathrm{~kW} / \mathrm{m}^{2}$ applied to one end of the bar throughout the duration of the simulation, and symmetry BCs are used for all other faces of the bar. The material properties of density $\rho=7832 \mathrm{~kg} / \mathrm{m}^{3}$, specific heat capacity $\mathrm{c}=434 \mathrm{~J} / \mathrm{kg} \cdot \mathrm{K}$, and thermal conductivity $\mathrm{k}=63.9 \mathrm{~W} / \mathrm{m} \cdot \mathrm{K}$ set to reflect a sample test problem available in [41]. The entire domain is initialized at a temperature of $\mathrm{T}_{0}=300 \mathrm{~K}$. The exact solution for this problem is shown below [41]:

$$
T(x, t)=\frac{2 q_{1}^{\prime \prime}(\alpha t / \pi)^{1 / 2}}{k} \exp \left(\frac{-x^{2}}{4 \alpha t}\right)-\frac{q_{1}^{\prime \prime} x}{k} \operatorname{erfc}\left(\frac{x}{2 \sqrt{\alpha t}}\right)+T_{0}
$$

where $\mathrm{q}_{1}^{\prime \prime}=-\mathrm{k} \partial \mathrm{T} /\left.\partial \mathrm{x}\right|_{\mathrm{x}=0}$ and $\alpha=\mathrm{k} / \rho \mathrm{c}_{\mathrm{p}}$ is the thermal diffusivity.

The sole design variable chosen for this optimization problem is the physically elapsed time since the application of the surface flux $\mathrm{BC}$, which is given a range of $\mathrm{t}=300 \mathrm{~s}$ to 
$\mathrm{t}=900 \mathrm{~s}$. The bounding values on the design variable will not necessarily be chosen as test points by the optimizer. The optimization tool is set to run for a total of 5 iterations of the Kriging algorithm, each with 5 distinct designs to be evaluated. The objective function for this test consists of maximizing the highest temperature within the domain at a given time. For this problem as time elapses with a prescribed heat flux applied to one end, the temperature in the domain should rise monotonically and the highest temperature within the domain should be located where the BC is applied. This means that to maximize the highest temperature in the problem domain, the optimal design returned by the optimization tool should be the largest elapsed time that is examined.

\section{Results}

When the VrSuite optimization tool is set to use Kriging, visualizations of the objective function value obtained for each design point examined for all design variables are produced, including graphical depiction of uncertainty in the value. The optimal objective function value for each Kriging iteration and for the entire design problem are also tracked.

Figure 4.2 depicts the evolution in the objective function values as the VrSuite optimizer runs multiple iterations, where the values of the sole design variable X1, representing the elapsed time, are found along the abscissa and the negative value of the objective function, the highest domain temperature at a given time multiplied by -1, is found along the ordinate. Each point represents an individual design. While counterintuitive, the objective function values shown are negative as the VrSuite optimization tool is coded to minimize the negative value of the objective function when the maximize option is chosen, resulting in the plots seen. As such, it is expected that longer elapsed times will yield more negative values for the objective function, which 

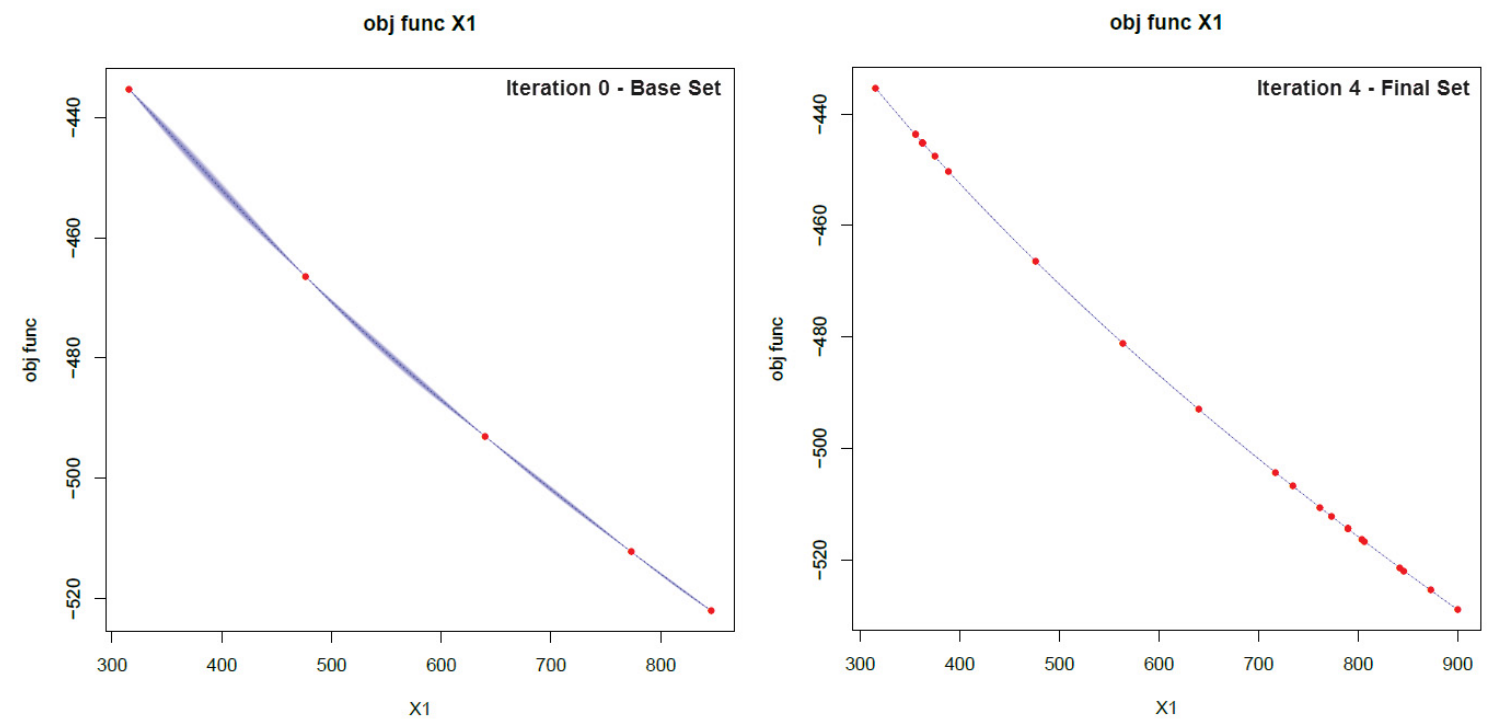

Figure 4.2: VrSuite optimization visualization for impact of design variable value on objective function value, with uncertainty.

physically correspond to a higher maximum domain temperature of equal magnitude. The left image contains the values for the initial set of design points chosen, which exhibit the expected trend. The band between points represents the uncertainty in the value of the objective function between the nodes, a visualization of the final benefit discussed in the preamble for Section 4.1. For subsequent iterations using the Kriging method, the VrSuite optimizer will chose values for the design variable to minimize this uncertainty. This is exemplified in the right image of Figure 4.2, a plot of objective function values for the final iteration, where many design points where chosen at the initial locations of uncertainty.
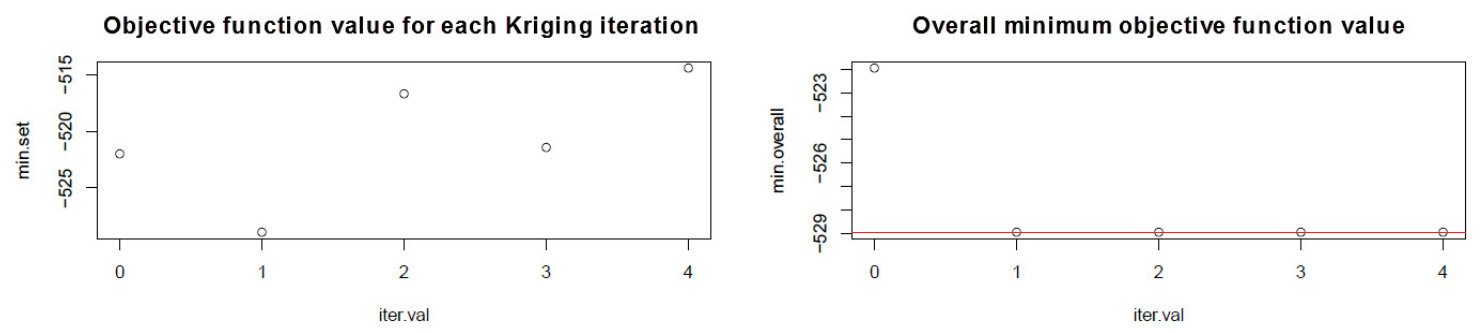

Figure 4.3: Best objective function value for each Kriging iteration (left) and objective function value for overall optimal design (right). 
Plots of the best objective function values are also produced. Figure 4.3 shows the evolution of the optimized objective function value over all Kriging iterations, with the left graph displaying the best value from each individual iteration and the right graph showing the overall best value from all iterations up until the current iteration. The latter provides some means to evaluate convergence, where little change in the overall minimum objective function value appears to occur after two iterations of the Kriging algorithm. The generated optimal design of an elapsed time of $t=872.9 \mathrm{~s}$ also matches expectations as it is the largest elapsed time simulated for this test.

Temperature Profiles for Semi-Infinite Bar Under Specified Flux BC

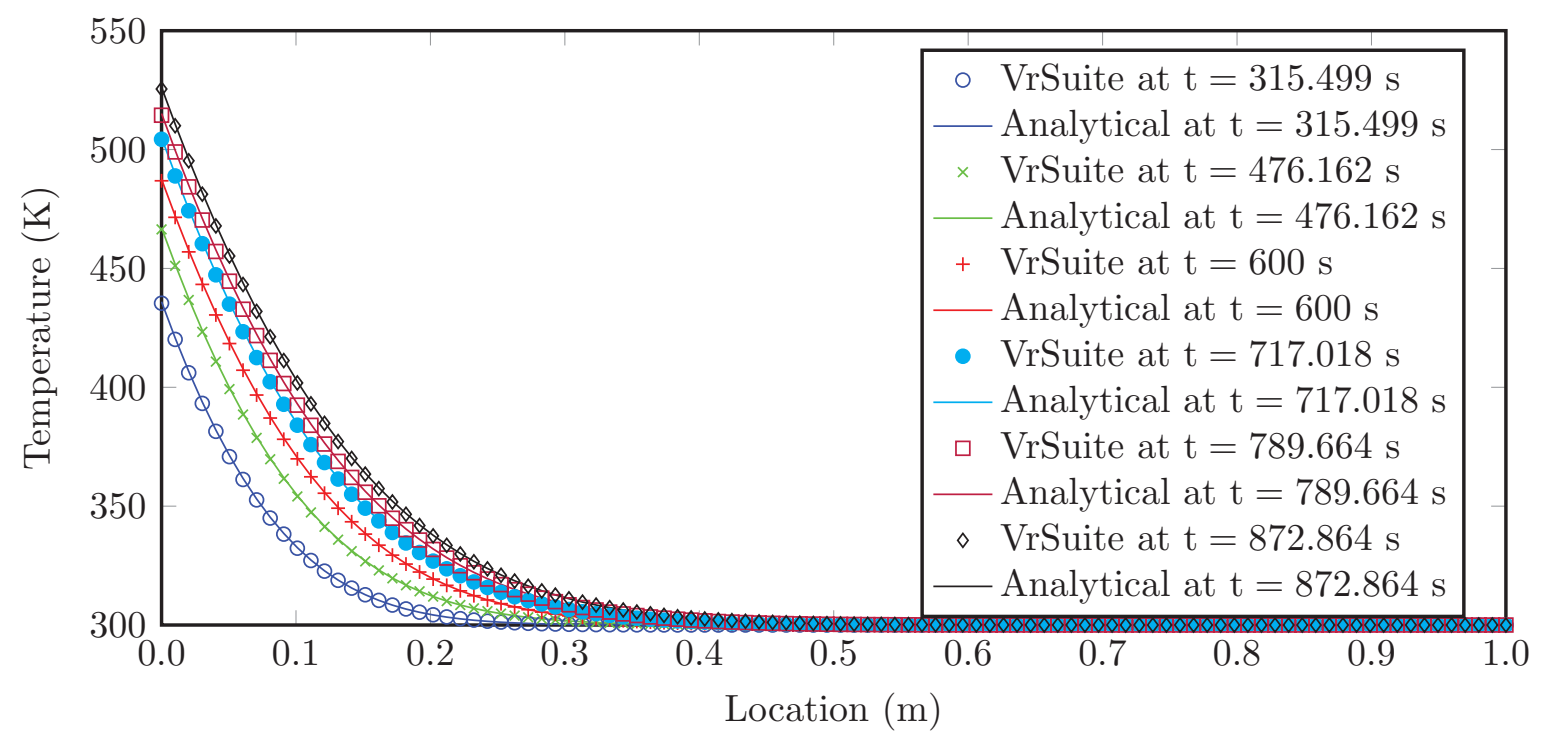

Figure 4.4: Simulated and analytical domain temperature profiles at six different elapsed times for the one-dimensional semi-infinite bar with prescribed Neumann BC.

To ensure the results are physically realistic, the simulated temperature profiles for some of the design points are compared to those generated using the analytical solution given by Equation (4.1). Examining Figure 4.4, the expected trend of increasing domain temperature with longer elapsed time is reflected by the domain temperature profiles examined at some of the designs chosen by the VrSuite optimizing tool. The 
simulated values also match the analytical solution extremely closely at all times studied, differing by no more than $0.15 \%$ relative difference at any location corresponding to a simulated data point, and it is also possible to claim that the semi-infinite condition is preserved given that the temperature at the end opposite where the heat flux is applied matches the initial domain temperature of $\mathrm{T}_{0}=300 \mathrm{~K}$. These results demonstrate that the VrSuite optimizer tool is functioning as intended, and that it is currently suitable to perform an initial optimization test for the MCHX technology.

\subsection{Initial MCHX Optimization - Number of Transfer Units Method}

Optimization of heat exchangers is usually based on economically-driven metrics, where an optimal design is that which offers the lowest operating costs through a minimum pressure drop compared to the alternatives. Optimization based on these metrics is readily described in literature, where the chosen objective function is composed of an overall depreciation on investment cost and operational costs [31]. For the MCHX technology, a different approach is chosen where the objective function outlined in Section 4.3.1 not only incorporates the usual economic factors, but is also influenced by the HXs ability to transfer heat effectively. This approach is used since selecting a design with minimal economic impact alone is not sufficient for nuclear applications where overall plant efficiency is a primary consideration.

While the operational costs can be determined once the pressure drop through the MCHX is solved, the heat transfer performance of the exchanger requires solution of dimensionless parameters, such as the Nusselt number $(\mathrm{Nu})$ or Prandtl number $(\mathrm{Pr})$, and knowledge of all operating conditions including outlet temperatures. For this 
optimization problem only the inlet conditions and geometry are specified, and the outlet conditions must be determined for each design in the DoE matrix. This is the same type of problem that was described in Section 2.2, which can be classified as a HX performance calculation. As such, the $\epsilon$-NTU method is once again suitable for the required analyses, and will be the basis of the model used by VrSuite for the initial MCHX optimization.

A description of the model is presented in this section, following an outline of the selected objective function. The results for this initial optimization of the MCHX technology are presented thereafter.

\subsubsection{Objective Function}

For the initial optimization of an MCHX, an objective function that incorporates heat transfer performance and losses due to friction is used, and all "other cost factors, i.e., equipment, maintenance and labor cost are not practically affected by the geometry" of the exchanger [34]:

$$
F=\eta_{N u}+\beta \eta_{f}
$$

Here $\eta_{\mathrm{Nu}}$ is a function that provides an amount of heat transfer augmentation with respect to that for a smooth surface and is defined below, with $\mathrm{Nu}$ representing the Nusselt number for the current design and $\mathrm{Nu}_{\mathrm{Sm}}$ being the value corresponding to an identical exchanger with smooth surfaces, given by the Dittus-Boelter correlation $\mathrm{Nu}_{\mathrm{Sm}}=0.023 \operatorname{Re}_{\mathrm{D}}^{0.8} \operatorname{Pr}^{0.3}[34]$ :

$$
\eta_{N u}=\left(\frac{N u}{N u_{S m}}\right)^{-1}
$$


The friction-based component of the objective function, $\eta_{\mathrm{f}}$, is defined in Equation (4.4), where $\mathrm{f}$ is the Darcy friction factor associated with the current combination of fluid flow conditions and channel geometry, and $\mathrm{f}_{\mathrm{Sm}}$ representing the value corresponding to an identical HX with smooth surfaces, equivalent to $\mathrm{f}_{\mathrm{Sm}}=64 / \mathrm{Re}_{\mathrm{D}}$

for a laminar flow or to $\mathrm{f}_{\mathrm{Sm}}=\left(1.8 \log _{10} \mathrm{Re}_{\mathrm{D}}-1.5\right)^{-2}$ for a turbulent flow [34]:

$$
\eta_{f}=\left(\frac{f}{f_{S m}}\right)^{1 / 3}
$$

Finally, $\beta$ is an economic weighting parameter that "accounts for the pumping cost to thermal energy cost and can be estimated using data concerning the cost of a unit of heat produced by natural fuel (e.g. natural gas) and the cost of the same amount of electric energy [34]." It typically takes on a value between 0 and 0.1 , and while the economic analysis required to select this value is beyond the scope of this thesis, a value of 0.03 is selected for this work to match that used by the authors of the previously cited paper. For the MCHX analysis, an optimal solution would be obtained through minimizing the objective function specified in Equation (4.2) [34].

\subsubsection{Design Evaluation Method}

In this analysis, only a single pair of identical square channels for hot and cold fluid streams is examined for each design, the operating pressures $\mathrm{P}_{\mathrm{h}}$ and $\mathrm{P}_{\mathrm{c}}$ are assumed to remain constant throughout the process, and water is chosen as the working fluid. For each experiment in the DoE matrix, a value for an objective function must be obtained in order to determine the merit of the corresponding design. In this initial optimization of an MCHX, the chosen objective function in Equation (4.2) requires solution of both a thermal-based function and a friction-based function. The required values, such as outlet temperatures, can be determined using the method outlined in Section 2.2.1 provided the geometry of the exchanger and the inlet conditions of the 
hot and cold fluid are specified. However, in most HX analyses using this method solution of the required parameters occurs using the bulk mean temperature of the fluid, or the average of $\mathrm{T}_{\mathrm{i}}$ and $\mathrm{T}_{\mathrm{o}}$ [41]. As such, the method utilized for this analysis is iterative since only $\mathrm{T}_{\mathrm{i}}$ is known initially.

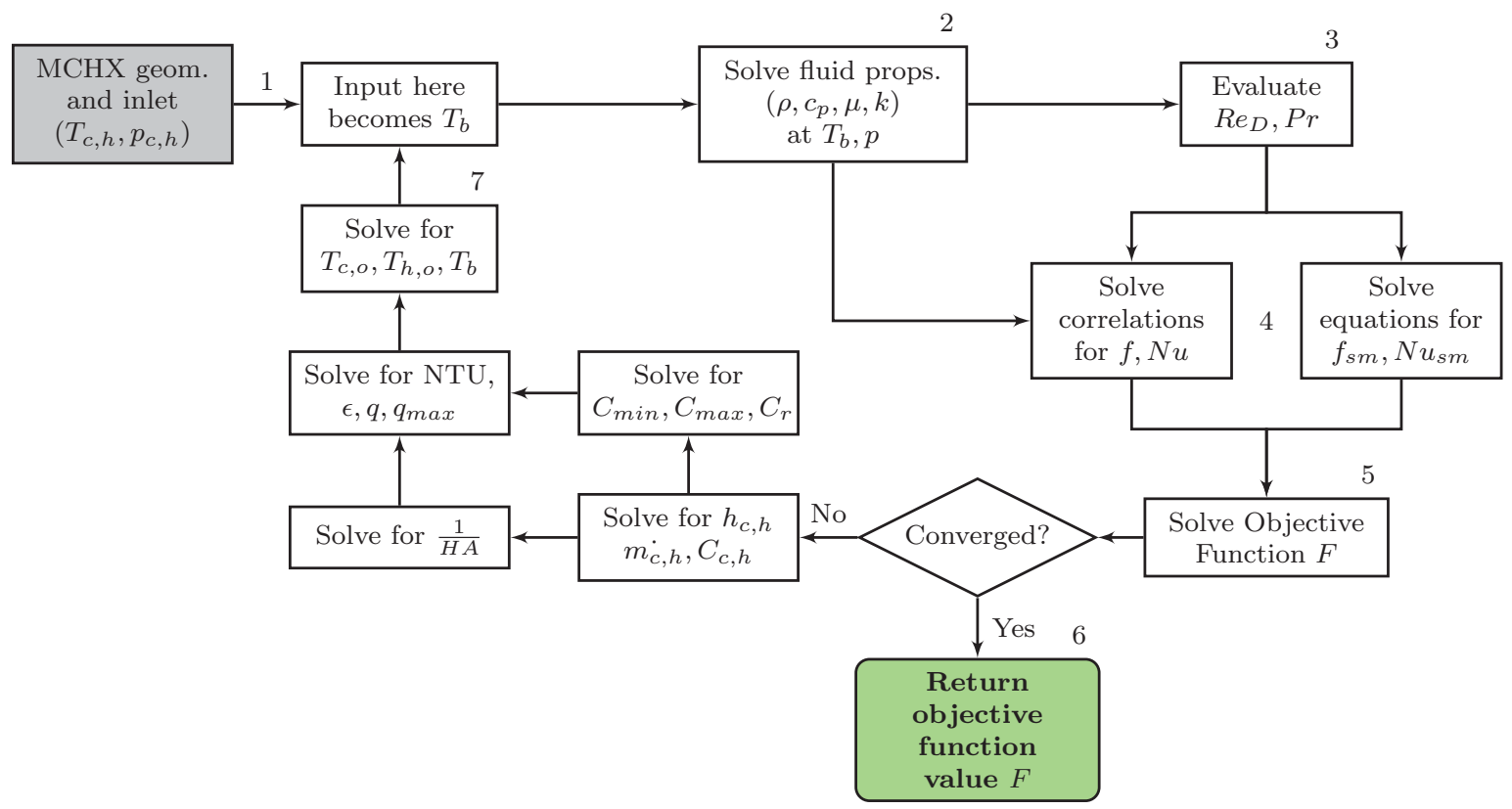

Figure 4.5: The solution procedure used for each experimental design of the MCHX, using the $\epsilon-\mathrm{NTU}$ method.

The solution procedure used is best described with the aid of the workflow diagram seen in Figure 4.5. In the first iteration the bulk fluid temperatures, denoted as $\mathrm{T}_{\mathrm{h}, \mathrm{b}}$ and $T_{c, b}$, are set to equal the inlet temperatures $T_{h, i}$ and $T_{c, i}$ respectively (1). Density, specific heat capacity at constant pressure, thermal conductivity, and dynamic viscosity are then evaluated for both fluids at their respective operating pressure and bulk temperature, using the thermophysical property tables that are readily available for water in the NIST database (2). The dimensionless parameters $\operatorname{Re}_{\mathrm{D}}$ and Pr are computed (3), allowing for solution of $\mathrm{f}$ and $\mathrm{Nu}$ using correlations that are available in the open literature (4). For example, measured friction factors for a wide 
range of operating conditions were correlated by Colebrook and are described by a transcendental expression [41]:

$$
\frac{1}{\sqrt{f}}=-2.0 \log _{10}\left[\frac{e / D_{h y}}{3.7}+\frac{2.51}{R e_{D} \sqrt{f}}\right]
$$

where e is the surface roughness and $\mathrm{D}_{\mathrm{hy}}$ is the hydraulic diameter. Additionally, a correlation provided by Gnielinski allows for solution of $\mathrm{Nu}$ for a large range of $\mathrm{Re}_{\mathrm{D}}$ in tubes, including the transition region [41]:

$$
N u_{D}=\frac{(f / 8)\left(\operatorname{Re}_{D}-1000\right) \operatorname{Pr}}{1+12.7(f / 8)^{1 / 2}\left(\operatorname{Pr}^{2 / 3}-1\right)}
$$

Both expressions in Equations (4.5) and (4.6) are valid for all operating conditions that would be encountered for any designs within the DoE matrix for the initial optimization of the MCHX technology. The equations seen in Section 4.3.1 are used to solve for the smooth surface friction factor and Nusselt number, $\mathrm{f}_{\mathrm{Sm}}$ and $\mathrm{Nu}_{\mathrm{Sm}}$.

With both the operating and smooth values for $\mathrm{f}$ and $\mathrm{Nu}$ computed, an objective function value $\mathrm{F}$ can be obtained for the current design (5). For any iteration other than the first, this value is compared to the previously obtained value and is considered to be converged when the percent difference between subsequent iterations is reduced past a specified convergence criteria. If the criteria is satisfied, $\mathrm{F}$ is returned (6). However, if it is the first iteration of the solution process or the criteria is not satisfied, the $\epsilon$-NTU method outlined in Section 2.2.1 is used to solve for hot and cold fluid outlet temperatures (7). For the overall heat transfer coefficient, H, a simple onedimensional thermal resistance model is used:

$$
\frac{1}{H A}=\frac{1}{\hbar_{h} A_{h}}+\frac{L_{\text {wall }}}{k_{\text {wall }} A_{\text {wall }}}+\frac{1}{\hbar_{c} A_{c}}
$$


where $\hbar$ is the convective heat transfer coefficient, $\mathrm{L}_{\text {wall }}$ is the wall thickness separating the hot and cold fluid, $\mathrm{k}_{\text {wall }}$ is the thermal conductivity of the HX wall, and A is the heat transfer area for that element of the thermal resistance model. Should the hot and cold channels be identical, as is the case for the analysis presented here, the heat transfer area for all elements will be equivalent. Once the overall heat transfer coefficient, effectiveness and NTU values, heat capacity rates, and heat transfer rates are all solved, the bulk fluid temperatures $T_{h, b}$ and $T_{c, b}$ can be calculated using the inlet and outlet temperatures and subsequently used for evaluation of fluid properties in the next iteration.

\subsubsection{Design Space and Optimization Setup}

For design problems like this initial optimization of an MCHX, it is typically desired to use a design space consisting of only a few of the most important parameters. This facilitates interpretation of the response of the objective function to the different variables and maintains a sufficiently low computational cost that is conducive to running multiple design iterations. While that approach is sound, there is some flexibility in this particular instance since the computational overhead is low, which is a result of having VrSuite run an independent executable that solves the HX problem using the $\epsilon-\mathrm{NTU}$ method detailed above instead of the default FEM solvers.

Consider the proposed problem of optimizing a simplified MCHX that is represented by a single pair of channels for a hot and cold fluid stream. The total number of design variables can be brought to a manageable level through a few simplifications. Letting both hot and cold channels have identical square cross-sections, inlet flow speeds, and operating pressures, the design can be characterized by the following variables: channel length $(\mathrm{L})$, channel width $(\mathrm{w})$, wall thickness between channels $\left(\mathrm{L}_{\mathrm{wall}}\right)$, and hot and cold inlet flow velocity (v). The inlet temperatures of each fluid and their 
operating pressure are specified to match the target design values for the Generation IV reactor designs, which at the time of writing are $\mathrm{T}_{\mathrm{c}}=350{ }^{\circ} \mathrm{C}, \mathrm{T}_{\mathrm{h}}=625{ }^{\circ} \mathrm{C}$, and $\mathrm{P}_{\mathrm{c}, \mathrm{h}}=25 \mathrm{MPa}[52]$.

The bounding values for each design variable, which are outlined in Table 4.1, are chosen somewhat arbitrarily as this problem is only meant to serve as an example for how this particular method can be used to perform an optimization. The bounds on the channel width and height are chosen to encompass a large range, with the lower end corresponding to the "micro" scale dimensions of the current MCHXs that are commercially available.

Table 4.1: Design variable bounds for the initial MCHX optimization using the $\epsilon-\mathrm{NTU}$ method.

\begin{tabular}{|c|c|c|}
\hline Design Variable & Minimum Value & Maximum Value \\
\hline \hline Channel Length, $L[\mathrm{~m}]$ & 0.5 & 15.0 \\
\hline Channel Width, $w[\mathrm{~m}]$ & 0.0005 & 0.075 \\
\hline Channel Wall Thickness, $L_{\text {wall }}[\mathrm{m}]$ & 0.0001 & 0.05 \\
\hline Fluid Inlet Velocity, $v[\mathrm{~m} / \mathrm{s}]$ & 0.1 & 10.0 \\
\hline
\end{tabular}

The remaining required parameters are summarized in Table 4.2, with values chosen somewhat arbitrarily. Once again, INCONEL alloy 600 or 690 are commonly used materials for HXs used in nuclear applications, having thermal conductivities of roughly $\mathrm{k}=15.0 \mathrm{~W} / \mathrm{m} \cdot \mathrm{K}$ and $\mathrm{k}=12.1 \mathrm{~W} / \mathrm{m} \cdot \mathrm{K}$ respectively for the temperature ranges chosen for this test $[42,43]$. An average of the two will be used for the initial optimization. The convergence criteria is the same as that discussed in Section 4.3.2, where the solver is deemed to be converged once the relative difference in the calculated value of the objective function for the current iteration compared to the previous 
iteration is less than the specified value. The VrSuite optimization tool is set to run 10 total iterations, evaluating 12 distinct designs in each iteration (three for each design variable) for a total of 120 designs.

Table 4.2: Additional solver parameters for the MCHX objective function solver using the $\epsilon-\mathrm{NTU}$ method.

\begin{tabular}{|c|c|}
\hline Solver Parameter & Value \\
\hline \hline HX Thermal Conductivity, $k[\mathrm{~W} / \mathrm{m} \cdot \mathrm{K}]$ & 13.55 \\
\hline Surface Roughness, $e[\mathrm{~m}]$ & 0.0005 \\
\hline Convergence Criteria & 0.00001 \\
\hline
\end{tabular}

\subsubsection{Results}

From the report generated automatically by VrSuite, the optimum design for the simplified MCHX problem can be determined by first looking at the plots of the objective function value for each Kriging iteration in Figure 4.6:
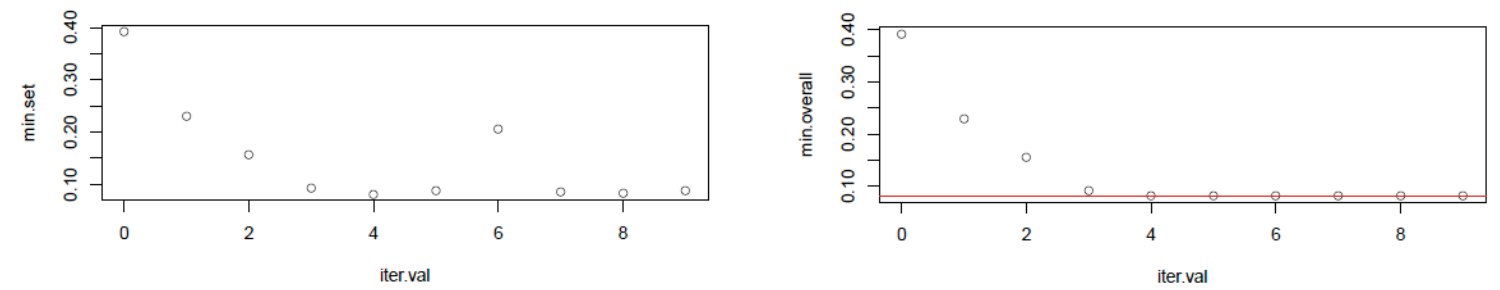

Figure 4.6: Minimum objective function value for each iteration (left) and over all iterations (right) for the MCHX optimization problem using the $\epsilon$-NTU method.

The left graph indicates the best objective function value from each Kriging iteration, whereas the right graph shows the overall best objective function value from all iterations as the solver computes solutions for each design within the DoE matrix. 
Noting once again that the best design corresponds to a minimum objective function value, the plots in Figure 4.6 indicate that the optimum solution was found during the $5^{\text {th }}$ Kriging iteration. The designs examined within this iteration are detailed in Table 4.3 .

Table 4.3: The portion of the DoE matrix corresponding to the $5^{\text {th }}$ Kriging iteration, adapted from the report generated by VrSuite.

\begin{tabular}{|c|c|c|c|c|c|}
\hline Run No. & $\mathbf{L}[\mathbf{m}]$ & $\mathbf{w}[\mathbf{m}]$ & $\mathbf{L}_{\text {wall }}[\mathbf{m}]$ & $\mathbf{v}[\mathbf{m} / \mathbf{s}]$ & Obj. Value \\
\hline \hline $\mathbf{4 9}$ & $\mathbf{3 . 2 9}$ & $\mathbf{0 . 0 2 2 6}$ & $\mathbf{0 . 0 0 0 1}$ & $\mathbf{3 . 9 0 9}$ & $\mathbf{0 . 0 8 1}$ \\
\hline 50 & 0.50 & 0.0224 & 0.0001 & 8.910 & 0.133 \\
\hline 51 & 15.0 & 0.0269 & 0.0001 & 6.506 & 1.055 \\
\hline 52 & 15.0 & 0.0296 & 0.0001 & 7.076 & 1.597 \\
\hline 53 & 15.0 & 0.0289 & 0.0001 & 5.298 & 1.549 \\
\hline 54 & 2.75 & 0.0291 & 0.0001 & 6.233 & 1.537 \\
\hline 55 & 7.64 & 0.0312 & 0.0001 & 5.759 & 1.970 \\
\hline 56 & 0.50 & 0.0312 & 0.0001 & 8.282 & 1.897 \\
\hline 57 & 5.42 & 0.0304 & 0.0001 & 6.993 & 1.751 \\
\hline 58 & 0.50 & 0.0328 & 0.0001 & 6.722 & 2.277 \\
\hline 59 & 0.50 & 0.0313 & 0.0001 & 6.157 & 2.037 \\
\hline 60 & 13.4 & 0.0327 & 0.0001 & 8.314 & 2.116 \\
\hline
\end{tabular}

The optimum design was examined in run number 49, providing an objective function value of $\mathrm{F}=0.081$ with a combination of channel length $\mathrm{L}=3.29 \mathrm{~m}$, channel width $\mathrm{w}=0.0226 \mathrm{~m}$, wall thickness between channels $\mathrm{L}_{\text {wall }}=0.0001 \mathrm{~m}$, and hot and cold inlet flow velocity $\mathrm{v}=3.909 \mathrm{~m} / \mathrm{s}$. What is immediately obvious from these results is that the solver has determined the optimum design has a wall thickness between the channels that corresponds to the lower bound on that parameter. This is expected as 
it would produce the best heat transfer properties for any configuration of the other design parameters based on the role it plays in determining the overall heat transfer coefficient via Equation (4.7). However, this computed value would yield an HX with walls that would not be able to withstand thermal stresses. This is a result of using an objective function that does not have any term that accounts for mechanical failure, which is certainly something that must be evaluated in future analyses. Some additional insight is provided by the plots of the objective function response to each of the individual design variables when the others are fixed at their optimal values.
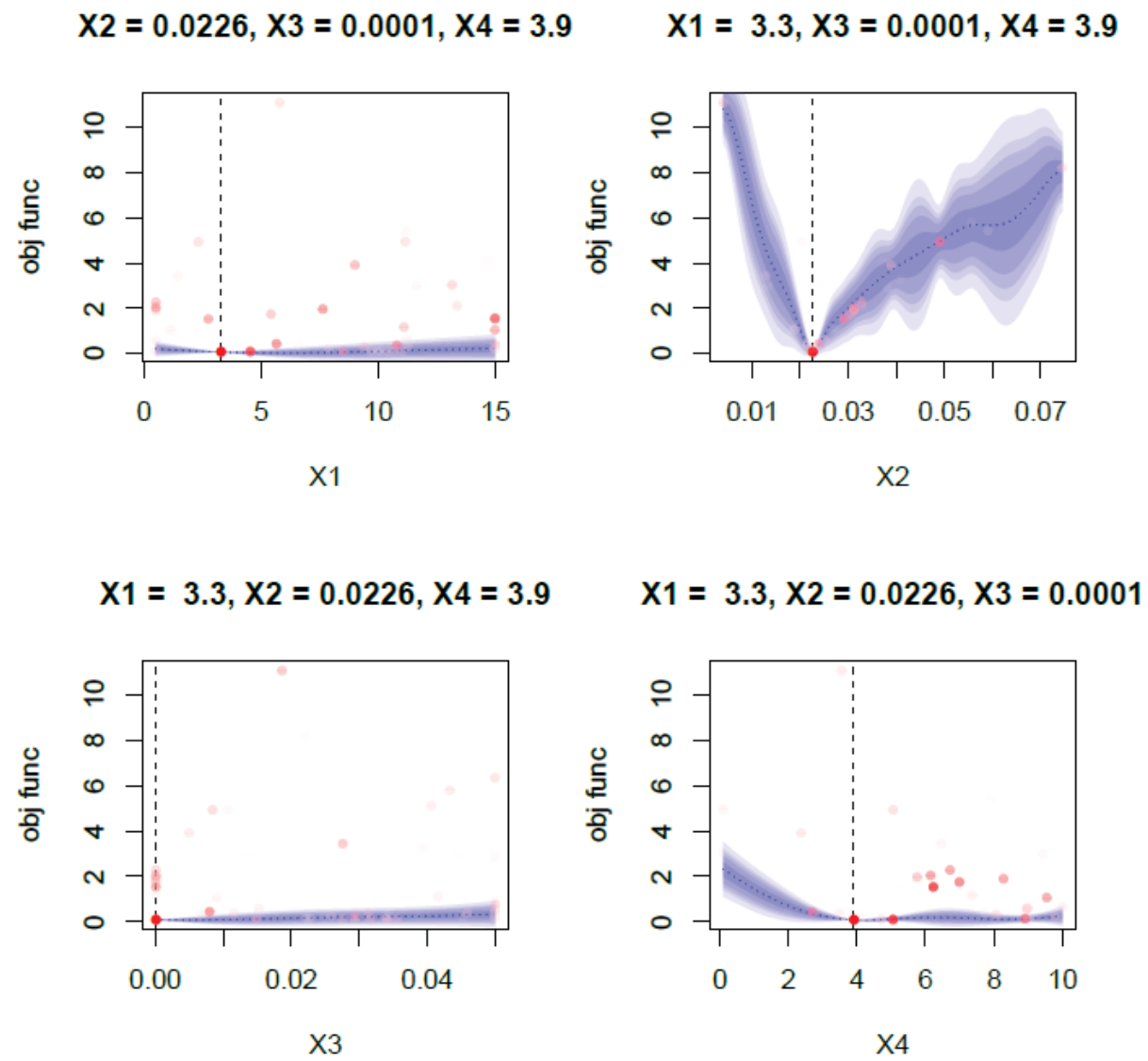

Figure 4.7: Objective function value response to all design variables in the $5^{\text {th }}$ Kriging iteration for the MCHX optimization problem using the $\epsilon$-NTU method.

In Figure 4.7, the design variables $\mathrm{L}, \mathrm{w}, \mathrm{L}_{\mathrm{wall}}$, and $\mathrm{v}$ are represented by $\mathrm{X} 1$ through $\mathrm{X} 4$ respectively. Once again, every data point on each graph represents the objective 
function value for an experiment from the DoE matrix, with the more vibrant points denoting designs from more recent Kriging iterations. The band represents the uncertainty in the objective function value at any location where there is no distinct design, and the dashed line denotes the expected objective function response in those locations.

From observation of the bottom left objective function response plot in Figure 4.7, it does not seem that the wall thickness has a very large impact on the objective function value, denoted by the very slight curve in the dashed line. This is a somewhat intuitive result as the wall thickness only exists as a part of one component within the expression for the overall heat transfer coefficient, which is not a dominant element in the model described in Sections 2.2.1 and 4.3.2. However it still shows a slope towards the lower bound, which is expected.

A similar trend can be seen in the plot for the length of the channel, in the top left of Figure 4.7, although the curve is slightly more pronounced. The channel length impacts both elements of the objective function provided by Equation 4.2, where heat transfer will be augmented by a longer channel with an increased heat exchange area, but the pressure drop that is incurred will be increased as well. The solver's choice of an optimal value that is closer to the lower bound would indicate that the pressure drop is the dominant factor. This would change should a different value be chosen for the economic weighting parameter $\beta$, where a larger value would increase the dominance of the pressure drop component.

The response generated by change in the channel length is also impacted by the design values used for the channel width and flow speed, and the solver's determination of the optimum values for these remaining parameters is not immediately obvious. 
Similar to the channel length, there appears to be a point of diminishing returns for increasing the inlet flow velocity. There is reasonably large improvement in value for the objective function as the flow speed is increased to the determined optimum value, but any further increase of this value does not seem to generate any notable improvements in the exhibited response, indicating that any augmentation in the heat transfer properties of the HX is negated by an increase in frictional losses. The optimal value falls once again near the lower bound of the problem, reinforcing the previous conclusion that the pressure drop is a dominant factor in determining the optimum design. Similar to response seen for the channel length, it is expected that change in the economic weighting parameter would adjust the importance of pressure drop in the analysis.

The top right plot in Figure 4.7 for the objective function response to change in the channel width shows that there is a very clear-cut optimum value within the specified design range. Given the prevalence of $\mathrm{Re}_{\mathrm{D}}$ within the equations used for the $\epsilon$-NTU method, it is not surprising that such an obvious optimal value would exist. Additionally, variation in w has a large impact on the objective function value with even small changes to the design value chosen, indicating that it is the dominant design variable of the four chosen for this initial analysis. It is interesting that the obtained optimum value for channel width of $\mathrm{w}=0.0226 \mathrm{~m}$ is quite a bit larger than the "micro" scale dimensions of the currently available MCHXs. This is most likely due to the idealizations used to simplify this test problem, and it is expected that evaluation of a more complete HX model, including realistic heat exchange between the HX to the surrounding environment, would yield significantly different results. 
While it was possible to obtain an optimized design, the results obtained for some of the design variables were not necessarily intuitive. The power of the Kriging optimization method is emphasized here given that the equations involved when employing the $\epsilon$-NTU method are both coupled and non-linear. Having the ability to obtain at least an initial estimate of an optimum design is particularly useful, especially in the early stages of the design process. Emphasis here should not be placed on the specific values obtained for the optimum design, but rather that this method can be used to determine the importance of certain design parameters, and on the ability to perform a search of the chosen design space for potential optimum designs that would be worthy of further investigation and analysis. This particular problem is also highly idealized, and examination of a more detailed HX model would also likely provide drastically different results.

Finally, it should be noted that the graphs displayed in Figure 4.7 show the response of the objective function to a single design variable while the entire design space is four-dimensional, and thus they cover only a very small fraction of a large space. As a future endeavour to improve coverage, a four-dimensional polynomial should be fit to the optimal point and the eigenvectors of the Hessian of this polynomial should be computed.

The report generated by VrSuite for this initial optimization of an MCHX using the $\epsilon-\mathrm{NTU}$ method can be found in Appendix B.

\subsection{Initial Expansion to Optimization using FEM}

While the model presented in Section 4.3 provides a good foundation for optimization of the MCHX technology, a fully transient, three-dimensional FEM analysis is 
the desired modelling method. However, the added complexity is not conducive for demonstrating the capabilities of the VrSuite optimization tool as a component of the method presented in this work. As an alternative, a simplified optimization problem using the VrSuite NS solver offers the opportunity to exhibit these capabilities, and show a method that can be expanded upon to include the fully coupled analysis at a later time. The model employed for this analysis uses the same mesh as that of the initial MCHX FEM performed in Chapter 2.

\subsubsection{Choice of Design Space and Objective Function}

To demonstrate the capabilities of VrSuite's optimization tool using Kriging with FEM modelling without introducing too much complexity, the design space has been limited to two variables: the channel hydraulic diameter and the inlet flow velocity. For simplicity, the hot and cold fluid inlet flow speeds are constrained to be equivalent, and the channels are identical and rectangular in shape. The bounds on channel size have been arbitrarily chosen to once again be close to "micro" scale given the initial nature of this analysis, in a similar vein to the reasoning used in Section 4.3.3. The lower and upper bounds for both the channel height and width have been selected to be $0.5 \mathrm{~mm}$ and $5 \mathrm{~mm}$ respectively. The optimizer is constrained to increment the size by no more than $1 \mathrm{~mm}$ per iteration. Similarly, the inlet flow velocity bounds are somewhat arbitrary, ranging from $1 \mathrm{~m} / \mathrm{s}$ to $5 \mathrm{~m} / \mathrm{s}$, with a max increment size of $1.5 \mathrm{~m} / \mathrm{s}$. It is intended to include a larger number of design variables for future analyses, but simplicity is desired here for demonstrative purposes. The channels are initialized with a hydraulic diameter of $\mathrm{D}_{\mathrm{hy}}=3.5 \mathrm{~mm}$ and the inlet flow speed of both fluids is set to $v_{i}=2 \mathrm{~m} / \mathrm{s}$. The optimization tool is set up to use three values for each

design variable in the base experimental set, and each of three total iterations of the Kriging method subsequently utilize three new values for each variable. 
To maintain simplicity, minimization of overall pressure drop $\Delta \mathrm{P}$ through the channels is chosen for the objective function. This function is not only sufficiently simple, but offers an intuitive solution via the Darcy-Weisbach equation for head loss [53]:

$$
\Delta P=f \cdot \frac{L}{D_{h y}} \cdot \frac{\rho v^{2}}{2}
$$

where $\Delta \mathrm{P}$ is the pressure drop, $\mathrm{f}$ is the Darcy friction factor, $\mathrm{L}$ is the channel length, $\mathrm{D}_{\text {hy }}$ is the hydraulic diameter, $\rho$ is the fluid density, and $\mathrm{v}$ is the fluid velocity. From Equation (4.8), it can be seen that the expected pressure drop is both proportional to the square of the flow velocity and inversely proportional to the hydraulic diameter, indicating that our optimal design should correspond to that which has the largest $\mathrm{D}_{\text {hy }}$ and lowest $\mathrm{v}$.

\subsubsection{Results}
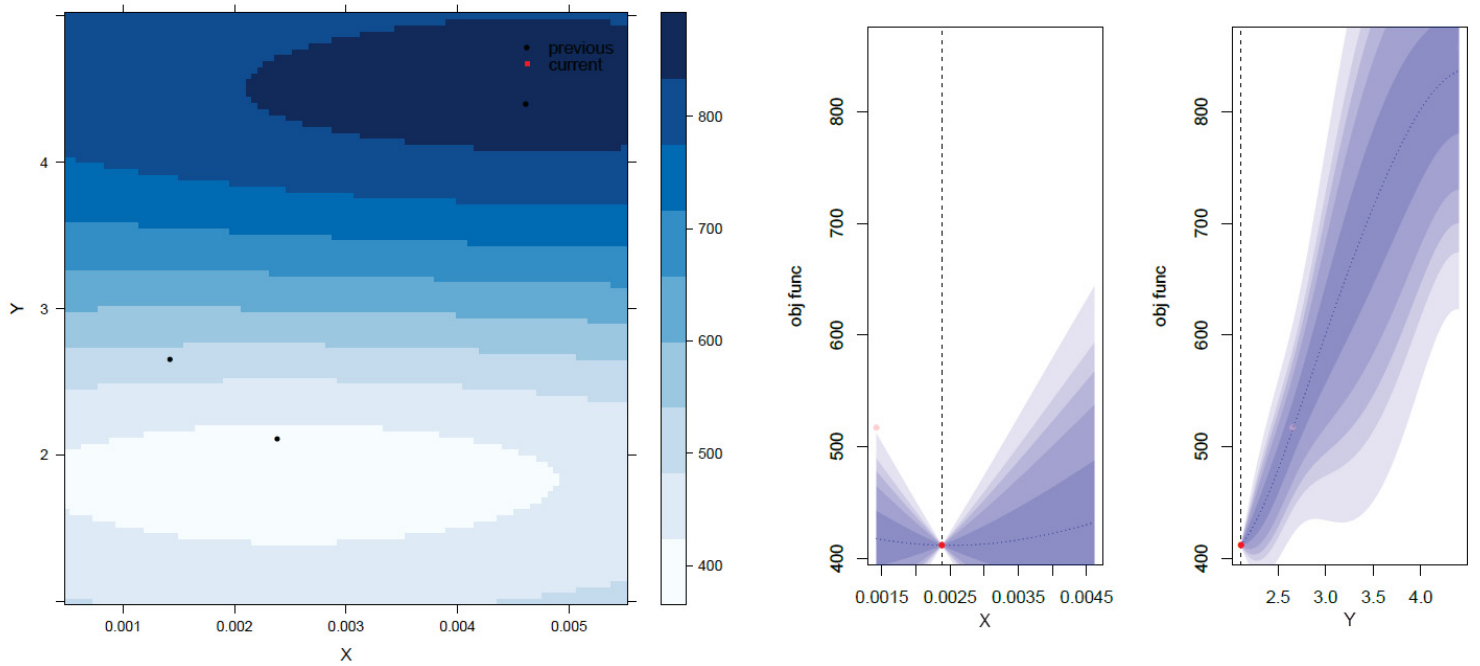

Figure 4.8: 2-D objective function response surface (left) and corresponding uncertainty for each design variable (right) for the first Kriging iteration.

When the optimizer is run for problems involving more than one design variable, the optimizer tool is capable of producing a two-dimensional response surface for the 
objective function, seen on the left of Figure 4.8, in addition to the usual plots of the uncertainty in the objective function value versus design variable value. This is particularly useful when examining only two design variables as their effect on the objective function value can be depicted simultaneously.

In Figure 4.8, $\mathrm{X}$ is representative of the channel hydraulic diameter and $\mathrm{Y}$ is the inlet flow velocity. After the first Kriging iteration, the optimization tool has determined that this system has a minimum pressure drop of $\Delta \mathrm{P}=411 \mathrm{~Pa}$ for design variable values of $\mathrm{D}_{\text {hy }}=2.38 \mathrm{~mm}$ and $\mathrm{v}_{\mathrm{i}}=2.11 \mathrm{~m} / \mathrm{s}$. While changes in the hydraulic diameter appear to have a minor effect on the pressure drop, increasing the flow speed appears to be the more significant factor, which is denoted by the sharp gradients in colour in the left image of Figure 4.8, and is the intuitive result. These results become more refined as the optimizer continues to solve, producing an "optimal" solution where a pressure drop of $\Delta \mathrm{P}=390 \mathrm{~Pa}$ is exhibited, corresponding to $\mathrm{D}_{\text {hy }}=5.00 \mathrm{~mm}$ and $\mathrm{v}_{\mathrm{i}}=1.00 \mathrm{~m} / \mathrm{s}$ for the fixed length of $\mathrm{L}=50.0 \mathrm{~mm}$.

This is the expected result from Equation (4.8) since $\Delta \mathrm{P}$ is proportional to the square of velocity and inversely proportional to the hydraulic diameter. Additionally, it is interesting to note that the design variable values designated as optimal by the solver are bounding values. It is expected that should the range be increased, the VrSuite optimization tool would choose larger hydraulic diameter and smaller inlet flow velocity values.

The two-dimensional objective function response surface, uncertainty plot for the inlet flow velocity, and convergence graph for the third of three Kriging iterations are shown in Figure 4.9. The trends observed previously appear to persist through the three iterations of the Kriging method, and it appears that the optimizer has 
reached a converged discrete solution. However, it would likely be beneficial to run the optimizer for a few more iterations to be certain of this conclusion.
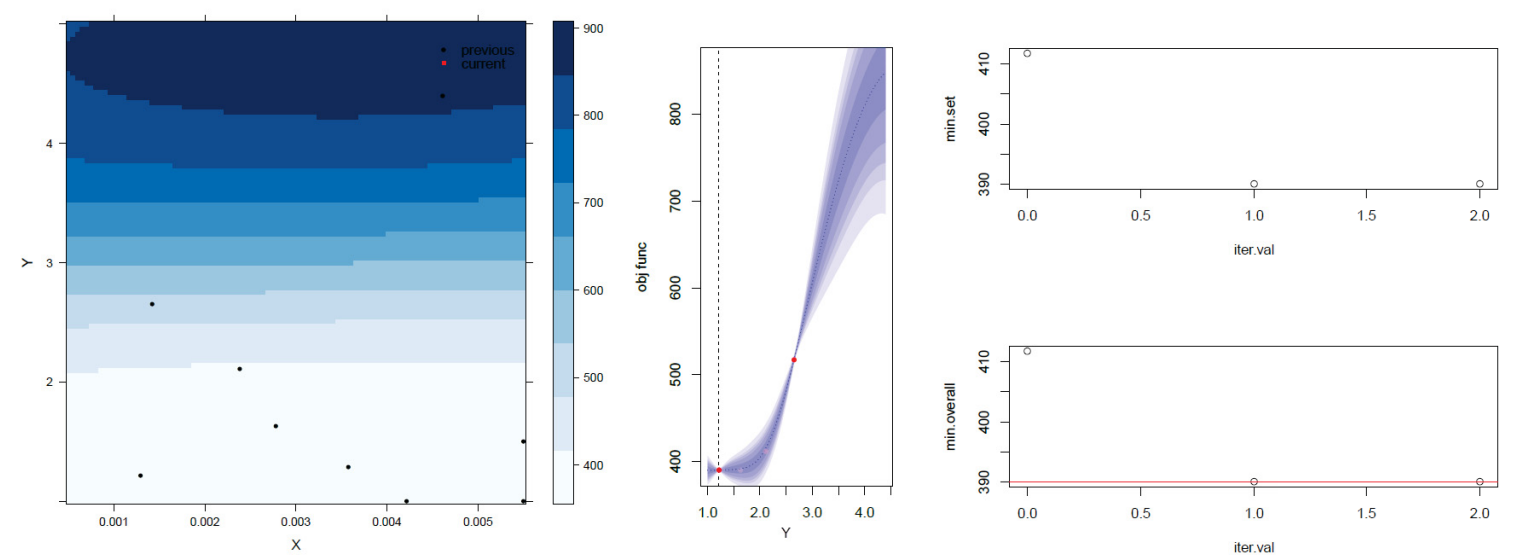

Figure 4.9: For the $3^{\text {rd }}$ iteration, the 2-D objective function response surface (left), uncertainty plot (middle), and optimizer convergence (right). 


\section{Chapter 5}

\section{Conclusions}

The objective of this work was to provide organization to the current MCHX research by demonstrating methods that can be used to perform analysis of potential microchannel heat exchanger designs. This was done successfully through creation of an accurate channel pair FEM model, generation of novel SPH simulations of free surface and two-phase flows with results that qualitatively represent the underlying physics, and determining an initial optimum MCHX channel pair design for input conditions selected to match the current Gen IV target values. Given the scarcity of design information for commercially available MCHXs in open literature, as well as the general exploratory nature of this work, these contributions are highly significant. Most notably, there is now an excellent foundation for future analyses of the MCHX technology.

The following sections present the major conclusions and specific results for each of the distinct methods examined, beginning with a review of the initial microchannel heat exchanger FEM modelling that was performed. Discussion of the most pertinent SPH models and the results from the first attempt at optimization of the technology follow, and a review of potential topics for further examination closes the chapter, and this thesis. 


\subsection{MCHX FEM Model Development and Analy- sis}

The first approach selected for analysis of the MCHX technology entailed use of the VrSuite software package and its FEM modelling capabilities to provide the framework for generation of an initial channel pair model. Although the requirements for an analysis and the elements that are included can yield FEM models that are quite complex, the method still offers a level of flexibility and ease of implementation that was attractive for the preliminary analysis discussed in Chapter 2.

Given that the initial analysis could be classified as a heat exchanger performance calculation, the $\epsilon$-NTU was utilized to generate outlet temperatures for both fluids, the total heat transfer rate, and the effectiveness for the modelled MCHX channel pair geometry. The method is commonly used for these types of HX problems, and comparison to the analytical results it can produce provided a means by which the simulated FEM results could be evaluated.

For the chosen geometry and inlet conditions, results produced by the VrSuite FEM simulations corresponded very well to those generated by the $\epsilon$-NTU method. At steady state, which occurred around a physical time of approximately 6 hrs, outlet temperatures for the cold and hot fluids of $\mathrm{T}_{\mathrm{c}, \mathrm{o}, \mathrm{sim}}=31.31{ }^{\circ} \mathrm{C}$ and $\mathrm{T}_{\mathrm{h}, \mathrm{o}, \mathrm{sim}}=68.54{ }^{\circ} \mathrm{C}$ were found. These values match those produced using the aforementioned method of $\mathrm{T}_{\mathrm{c}, \mathrm{o}, \text { ana }}=31.50^{\circ} \mathrm{C}$ and $\mathrm{T}_{\mathrm{h}, \mathrm{o} \text {,ana }}=68.37^{\circ} \mathrm{C}$, and comparison of the total temperature changes in either fluid yields relative differences of no more than $2.92 \%$. A similarly reasonable comparison exists for the obtained heat transfer rates and effectiveness', with there being only $2.30 \%$ relative difference between $\mathrm{q}_{\text {sim }}=200.3 \mathrm{~W}$ and $\mathrm{q}_{\mathrm{ana}}=205.0 \mathrm{~W}$ and only $3.01 \%$ between $\epsilon_{\mathrm{sim}}=0.129$ and $\epsilon_{\mathrm{ana}}=0.133$. 
Examination of the simulated temperature profiles for both the cold and hot fluid also appear to indicate that heat is being transferred from the hot fluid, through the MCHX solid domain, and into the cold fluid as intended. The shape of the profiles mimic the expected trends for the counterflow configuration selected, although slightly sharper temperature gradients exist near the fluid inlets. A quick mesh sensitivity study shows that even a drastic increase in total element count from 1680 to 15600 8-node volume elements produces negligible changes in either the total heat transfer rate or $\mathrm{HX}$ effectiveness for the examined MCHX channel pair configuration. As such, use of the initial model for expansion of the optimization problem presented in Chapter 4 was recommended, especially considering that a fully-transient, threedimensional, coupled analysis could be performed in approximately 1.81 hrs of total computation time.

Despite the excellent match seen in the simulated results and those produced using the $\epsilon-N T U$ method, there are some notable limitations to the initial model. Modelling of only a single HX channel pair is the most notable, and examination of a more detailed model such as one including an entire array of channels is recommended. Inlet plenums for either fluid are not currently modelled, where inclusion of those features would likely improve the accuracy of the results and would facilitate capturing of any existing entrance effects. The slightly sharp gradients in the fluid temperature profiles might also be smoothed with the addition of the inlet plenums.

Regardless, the preliminary MCHX model still accomplishes the desired goal of showcasing how FEM can be used as an analysis method for this particular problem. It bears repeating that the results produced are only preliminary, and that the method used is what should be focused on. Even still, the excellent agreement found between the simulated results and those found using the $\epsilon$-NTU method suggested that the 
model is replicating an MCHX channel pair acceptably for a very preliminary model. The model was also deemed acceptable for use as the basis for the initial optimization using the FEM method for similar reasoning.

\subsection{Use of Smoothed Particle Hydrodynamics}

With the initial MCHX FEM model successfully developed the research focus shifted towards use of smoothed particle hydrodynamics, an alternative analysis method that offers the ability to examine problems with complex phenomena. More specifically, the possibility of boiling flows within the MCHX channels exists and use of the SPH framework greatly facilitates the resolution of flows with sharp discontinuities in material properties or moving phase boundaries. Such problems would be very difficult to solve using traditional fixed-mesh FEM, which emphasizes the importance of the SPH method and provided a motive for examining the capabilities of the method in this work.

Demonstration of the method was accomplished through presentation of a number of problems ranging from simple fluid flow or heat transfer models to a heated channel model that incorporated boiling, beginning with an examination of the VrSuite SPHNS solver. Results that qualitatively simulate the observed physics were obtained for a free surface flow model of a drop falling into a fixed basin, including the expected oscillatory motion that would occur for the particles that did not jump over the side walls upon initial contact. Similarly plausible flow was also exhibited in the model that incorporated a particle inlet and outlet, where injection and removal of particles from the solution domain were simulated successfully. The occurrence of what appeared to be an eddy-like flow structure downstream of the backwards-facing step in the model was also particularly encouraging despite the low resolution, an 
issue that would be rectified with reduced particle size and spacing while increasing particle count. In both cases, the obtained results appear to replicate those that can be found in other sources successfully.

Application of the VrSuite SPH thermal solver to model the Stefan problem generated results that agree strongly with the analytical solution, where the simulated data points differed by no more than $5.66 \%$ from their respective analytical values. The location of the solidification front at an elapsed time of $\mathrm{t}=0.0568 \mathrm{~s}$ was determined by the software to be $\mathrm{x}_{\mathrm{sim}} \approx 0.225$, which compares very favourably to the analytical value of $\mathrm{x}_{\mathrm{sim}} \approx 0.2383$, differing by only $5.6 \%$. A significant result from this test problem is certainly the ability of the method to effectively resolve phase change within stationary particles, which represented a key step in creating a foundation for the MCHX modelling efforts.

The heat transfer problem was further expanded upon to models involving both the SPH NS and thermal solvers. In the pool boiling model, application of heat to the bottom of the pool directly caused the adjacent fluid particles to experience phase change to vapour, denoted by a distinct change in the volume around them. While the fluid domain was not subjected to any flow BCs, observation of motion throughout caused by the expansion of the vapour bubbles is significant and indicates successful coupling the two SPH solvers. Expansion to a model of flowing water through a channel with heated walls produced similarly plausible bubble nucleation and expansion within the moving fluid, an accomplishment that is both novel and significant considering the difficulties that would be posed if traditional FEM were used. The results generated from both of the models using coupled SPH-NS and SPH thermal solvers suggest that simulation of boiling flows within a more complex MCHX model is probable. 
Although the analyses presented were preliminary, the results generated by all models appeared to be qualitatively simulating the observed physics and suggest that the solvers are functioning appropriately. The purpose of creating these SPH models was simply to demonstrate the ability to model more complex free surface flows using the method, and to establish it as a tool that can be used should the possibility of multiphase flow conditions within the heat exchanger exist.

\subsection{Optimization Method for the MCHX}

To augment the selection of available design tools discussed, namely traditional fixedmesh FEM for quick analyses and application of smoothed particle hydrodynamics for problems with more complex flow phenomena, the Kriging optimization method provides the designer with the ability to efficiently explore a design space to generate knowledge of parameter interactions or search for optimal designs. VrSuite offers the ability to solve optimization problems using the Kriging method, which was successfully demonstrated for both a simple test problem and for the initial optimization of the MCHX technology.

The classical test problem of one-dimensional conduction into a semi-infinite solid was expanded upon to become a simple optimization problem. Using the elapsed time since application of a fixed Neumann BC to one end of the domain as the sole design variable, the VrSuite optimization tool was used to determine an optimum "design" that would yield the greatest maximum domain temperature. The optimal design generated by the solver used an elapsed time of $t=872.9 \mathrm{~s}$, the expected result given that it was the largest time examined within the provided bounds on the sole design variable. Examination of the temperature profiles within the solid domain at a number of times also showed very good agreement with the analytical 
solution for the problem, exhibiting no more than a $0.15 \%$ relative difference at any location corresponding to a simulated data point, indicating that the optimization tool is certainly functional.

Application of Kriging-based optimization via the $\epsilon$-NTU method to the MCHX was also successfully demonstrated, while utilizing an objective function that incorporated not only the usual economic factors but also a term for heat transfer performance. For the given bounds on the selected design parameters, the channel pair geometry and inlet flow velocity, the computed optimal design provided an objective function value of $\mathrm{F}=0.081$ using a combination of channel length $\mathrm{L}=3.29 \mathrm{~m}$, channel width $\mathrm{w}=0.0226 \mathrm{~m}$, wall thickness between channels $\mathrm{L}_{\text {wall }}=0.0001 \mathrm{~m}$, and hot and cold inlet flow velocity $\mathrm{v}=3.909 \mathrm{~m} / \mathrm{s}$.

As expected, the optimal value for the wall thickness between channels corresponds to the lower bound on that design parameter. However, it should be noted that use of an objective function that does not account for mechanical failure produced an optimum value for the wall thickness that would not be able to withstand thermal stresses. This could be rectified with appropriate adjustments to the utilized objective function, although it is expected a different optimum value for that design parameter would be produced. Additionally, the sensitivity of the objective function value to changes in $\mathrm{L}_{\text {wall }}$ appear to be minimal.

A similar trend in the objective function response to changes in the channel length is also seen, with the optimal value falling within the lowest quarter of the specified range provided for that design variable. Changes in the channel length impacts both elements of the objective function, but the location of the optimal value suggests that the component driven by the pressure drop is the dominant factor. Granted, this 
element of the objective function response is directly tied to the economic weighting parameter $\beta$ and choice of a different value would strongly influence the obtained results.

Increasing in the inlet flow speed from the lower bound shows distinct improvement until the determined optimal value is reached. However there appears to be a point of diminishing returns for any further increases where improvement in the heat transfer characteristics are offset by the larger frictional losses, supporting the earlier claim that the pressure drop term in the objective function tends to dominate the overall response for the given parameters. Additionally the objective function shows the most defined and drastic response to variation in the channel width, indicating that it is the dominant design variable for this initial examination. Interestingly, the optimal channel width of $\mathrm{w}=0.0226 \mathrm{~m}$ is substantially larger than the "micro" scale dimensions of the commercial MCHXs that are currently available, which is most likely a result of the modelling idealizations used for this initial application of the Kriging optimization method.

To demonstrate application of the same optimization method to the MCHX FEM model developed in Chapter 2, a simplified version of the problem using only the FEM NS solver was presented. Given the dominance of the economic term in the previously utilized objective function, the selected objective function was limited to the pressure drop alone to maintain simplicity. With the inlet flow speed and channel hydraulic diameter also selected as the only design variables, a the VrSuite optimization tool determined that a minimum pressure drop of $\Delta \mathrm{P}=390 \mathrm{~Pa}$ could be obtained using design values of $D_{\text {hy }}=5.00 \mathrm{~mm}$ and $\mathrm{v}_{\mathrm{i}}=1.00 \mathrm{~m} / \mathrm{s}$. Both of the optimal design values also correspond to bounding values for those parameters, an expected result that bolsters confidence that the optimization tool is functioning as 
intended and that the method can be used for more complex versions of the MCHX design problem than that presented in this work.

\subsection{Recommendations for Future Work}

The majority of the observations and conclusions presented in this work result from examination or use of very preliminary models for an MCHX, with the intent of demonstrating methods that can be used to perform the desired analyses. As such, there exist a number of notable modelling adaptations or adjustments to the approaches used that would either address some of the discrepancies in the presented results or improve the overall fidelity of the models. Some suggestions for possible enhancements that future research directly associated with this work could examine include, but are certainly not limited to, that which follows.

The initial FEM model presented in Chapter 2 consist of only a single pair of channels, which was done to ensure simplicity for the preliminary analysis shown. Expanding this model to include multiple MCHX plates with numerous channels on each and including heat transfer with the environment surrounding the entire HX, similar to the level of complexity displayed on the left side of Figure 2.2, would provide a more accurate assessment of the performance of the HX design. Additionally, the only channel shape modelled in this work is a square channel for both working fluids. Examination of the effectiveness of a HX design utilizing different channel geometry for either one or both of the hot and cold fluid channels would provide valuable insight. Development these new models would also support integration of channel geometry as an additional design parameter for future optimization attempts. The overall fidelity of the generated models could also stand to be improved upon through successive increases in the level of detail incorporated as the models become more 
refined. As examples, examination of the effect of channel fouling on the performance of the heat exchanger or running analyses with fully coupled VrSuite FEM thermal, $\mathrm{NS}, \mathrm{AD}$, and stress solvers as discussed in Section 2.1.2 to evaluate the impact of channel deformation should be performed.

The models presented using the SPH method offer similar room for improvement, with performing a more thorough evaluation of the VrSuite SPH-NS solver having the highest priority. The results discussed are primarily qualitative in nature as the software functionality to extract data that would allow for proper analysis and verification of the solver had not been implemented at the time of writing. Once the ability to extract data such as time-averaged velocity profiles at a location within a channel exists, performing verification of the existing channel model through a quantitative comparison with known solutions for the same type of flow and by examining the heat transfer that occurs from heated walls to the fluid should occur.

Once completed, the heated channel flow model should be expanded upon to become a single MCHX channel pair for a hot and cold fluid, perhaps using a particle discretization similar to that shown in Figure 5.1. The coarser set of particles represents the MCHX solid, while the finer discretization of particles is for the two fluids. Use of the SPH method would allow for examination of a channel pair that potentially contains multiphase fluids, and a direct comparison of the obtained results to those generated using the FEM model described in Chapter 2 could be performed. Furthermore, all of the SPH models presented have been created to be only a single layer "thick" to reduce complexity of the problems used for an initial verification of the software, which differs from a three-dimensional analysis by a single global scalar constant. Examination of a fully three-dimensional SPH model of an MCHX channel pair, or larger grouping of channels similar to the larger MCHX FEM model 


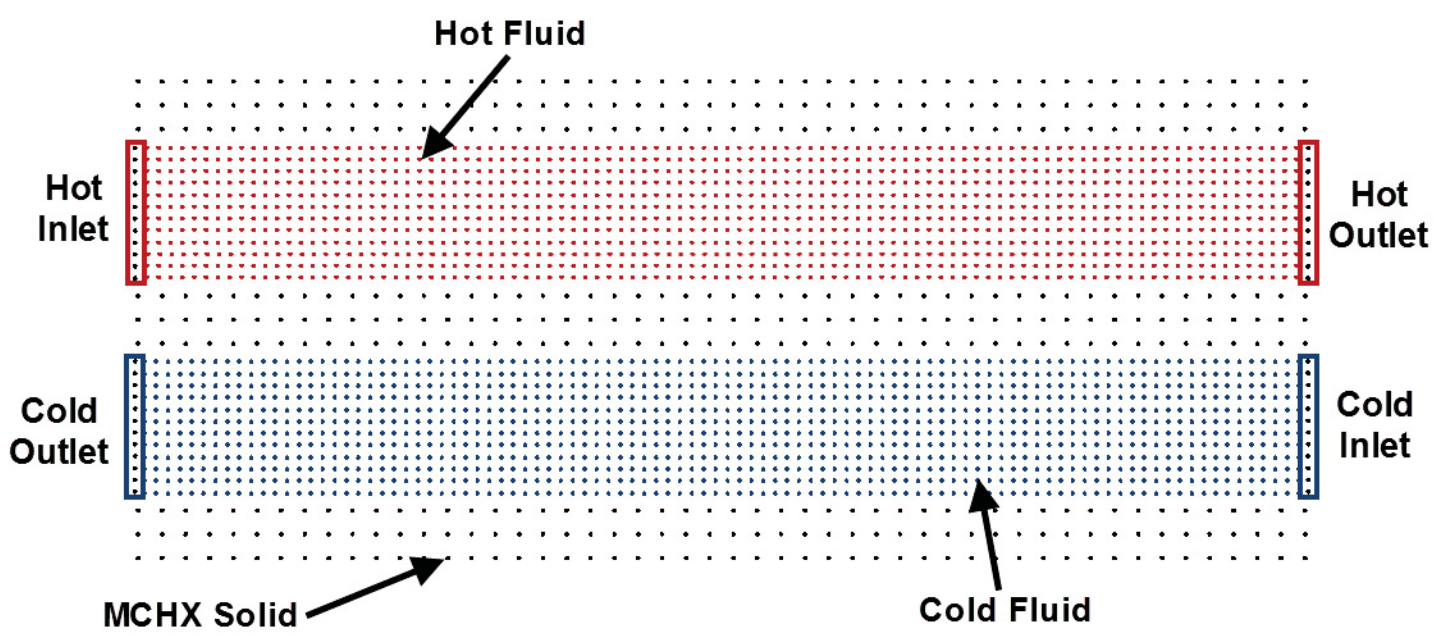

Figure 5.1: A potential SPH particle discretization that can be used to model an MCHX channel pair.

of multiple plates suggested above, is of interest. Finally, interface thermodynamics were not taken into account for any problems involving phase change, which should be addressed going forward.

As with the analyses presented using FEM and the SPH method, the initial optimization of an MCHX design that was detailed in Chapter 4 is very much a preliminary demonstration of the capability of the Kriging method to be used for a problem of this type. The problem presented had been greatly simplified to use only four design variables, and performing a similar optimization using a greater number could drastically alter the determined optimal design. Specifically, distinct channel geometry and inlet flow conditions are suggested as a starting point. The objective function utilized for the initial optimization problem also does not account for the possibility of rupturing channels. It is critical that there is no mixing of the hot and cold side fluids, and any design within the DoE matrix that will rupture due to stresses caused by high thermal gradients should be immediately rejected regardless of the objective function value obtained. The current "optimum" value for the wall thickness between 
channels of $\mathrm{L}_{\text {wall }}=0.0001 \mathrm{~m}$ is extremely small and would not be able to handle the thermal stresses that would be encountered during operation. Use of a modified objective function is expected to rectify this. Additionally, another simplified problem was presented in Section 4.4 that only used the VrSuite FEM NS solver. While the reasoning to use only the one solver was predominantly for simplicity, the software is currently unable to run optimization problems that use coupled solvers. Furthering of the FEM-based optimization detailed there to include the other solvers is strongly suggested once the capability to do so is implemented, allowing for comparison to the results obtained using the $\epsilon$-NTU method as the basis for optimization. It would also be beneficial to run experiments to verify the output of each optimization problem to hopefully confirm that the designs are optimal. 


\section{References}

[1] M. Schneider and A. Froggatt et al. World nuclear industry status report 2013. Online Document, Jul 2013. [Cited 7 Jul 2014]. Available: http://www.worldnuclearreport.org/IMG/pdf/ 20130716msc-worldnuclearreport2013-lr-v4.pdf.

[2] International Atomic Energy Agency. Nuclear power capacity trend. Online Document, 3 Jul 2014. [Cited 7 Jul 2014]. Available: http://www.iaea.org/ PRIS/WorldStatistics/WorldTrendNuclearPowerCapacity . aspx.

[3] U.S. Census Bureau. World population. Online Document, 19 Dec 2013. [Cited 30 Jun 2014]. Available: http://www.census.gov/population/international/ data/worldpop/table_population.php.

[4] World Nuclear Association. World energy needs and nuclear power. Online Document, Jun 2014. [Cited 30 Jun 2014]. Available: http: //www . world-nuclear.org/info/Current-and-Future-Generation/ World-Energy-Needs-and-Nuclear-Power.

[5] World Nuclear Association. Comparison of lifecycle greenhouse gas emissions of various electricty generation sources. Online Document, Jul 2011. [Cited 30 Jun 2014]. Available: http://www.world-nuclear.org/uploadedFiles/org/wNA/ Publications/Working_Group_Reports/comparison_of_lifecycle.pdf.

[6] U.S. Energy Information Administration. Levelized cost and levelized avoided cost of new generation resources in the annual energy outlook 2014. Online Document, 17 Apr 2014. [Cited 7 Jul 2014]. Available: http://www.eia.gov/ forecasts/aeo/electricity_generation.cfm.

[7] M. Ohadi et al. Next Generation Microchannel Heat Exchangers. Springer Science \& Business Media, New York, NY, USA, 2012. 
[8] J. McLellan et al. An introduction to the use of smoothed particle hydrodynamics in the modelling and optimization of a microchannel heat exchanger. In 2014 Canada-China Conference on Advanced Reactor Development (CCCARD-2014), Niagara Falls, ON, Canada, 27-30 Apr 2014.

[9] D. Southall and S.J. Dewson. Innovative compact heat exchangers. In Proceedings of ICAPP '10, San Diego, CA, USA, 13-17 Jun 2010.

[10] J.E. Hesselgreaves. Compact Heat Exchangers: Selection, Design and Operation. Elsevier Science Ltd., Kidlington, Oxford, UK, 2001.

[11] M.G. Khan and A. Fartaj. A review on microchannel heat exchangers and potential applications. International Journal of Energy Research, 35:553-582, Jul 2011.

[12] A. Shirzadi. Diffusion bonding. Online Document, 01 Nov 2011. [Cited 8 Jul 2014]. Available: http://www.msm.cam.ac.uk/phase-trans/2005/Amir/ bond.html.

[13] Heatric Ltd. The benefits of deploying heatric exchangers: Performance. Online Document, Jul 2014. [Cited 10 Jul 2014]. Available: http://www.heatric.com/ superior_performance.html.

[14] DA Reay. Compact heat exchangers: A review of current equipment and R\&D in the field. Heat Recovery Systems and CHP, 14:459-474, Sept 1994.

[15] M.M.A Bhutta et al. CFD applications in various heat exchangers design: A review. Applied Thermal Engineering, 32:1-12, Sept 2011.

[16] S.P. Kar. Cfd analysis of printed circuit heat exchanger. Master of technology - mechanical engieering, National Institute of Technology Rourkela, Rourkela, Odisha, India, 2007.

[17] J. Teng et al. Fluid Dynamics, Computational Modeling and Applications. InTech, Rijeka, Croatia, 2012.

[18] L. Zhang et al. Measurements and modeling of two-phase flow in microchannels with nearly constant heat flux boundary conditions. Journal of Microelectrical Systems, 11:12-19, Feb 2002.

[19] C.H. Oh et al. Desing option of heat exchanger for the next generation nuclear plant. Journal of Engineering for Gas Turbines and Power, 132:1-9, Mar 2010. 
[20] K. Shirvan et al. Investigation of high-temperature printed circuit heat exchangers for very high temperature reactors. Journal of Engineering for Gas Turbines and Power, 131:1-7, Nov 2009.

[21] K. Shirvan et al. The design of a compact integral medium size PWR. Nuclear Engineering and Design, 243:393-403, Nov 2011.

[22] J. Van Meter. Experimental investigation of a printed circuit heat exchanger using supercritical carbon dioxide and water as heat transfer media. Master of science, Kansas State University, Manhattan, KS, USA, 2008.

[23] K. Natesan et al. Preliminary issues associated with the next generation nuclear plant intermediate heat exchanger design. Journal of Nuclear Materials, 392:307315, Mar 2009.

[24] D.J. Price. Smoothed particle hydrodynamics and magnetohydrodynamics. Journal of Computational Physics, 231:759-794, Feb 2012.

[25] S. Nugent and H.A. Posch. Liquid drops and surface tension with smoothed particle applied mechanics. Physical Review E, 62:4968-4975, Oct 2000.

[26] P.W. Cleary. Modelling confined multi-material heat and mass flows using SPH. Applied Mathematical Modelling, 22:981-993, Feb 1998.

[27] J.J. Monaghan. Solidification using smoothed particle hydrodynamics. Journal of Computational Physics, 206:684-705, Feb 2005.

[28] G. Box and P. Liu. Statistics as a catalyst to learning by scientific method part i - an example. Journal of Quality Technology, 31:1-15, Jan 1999.

[29] G. Box. Statistics as a catalyst to learning by scientific method part i - discussion. Center for Quality and Productivity Improvment Report, 172:1-13, Jun 1999.

[30] D.R. Jones. A taxonomy of global optimization methods. Journal of Global Optimization, 21:345-383, 2001.

[31] H.J. Fontein and J.G. Wassink. The economically optimal design of heat exchangers. Engineering and Process Economics, 3:141-149, 1978.

[32] A.C. Caputo. Heat exchanger deisgn based on economic optimisation. Applied Thermal Engineering, 28:1151-1159, Sept 2008. 
[33] A. Hadidi and A. Nazari. Design and economic optimization of shell-and-tube heat exchangers using biogeography-based (BBO) algorithm. Applied Thermal Engineering, 51:1263-1272, Dec 2012.

[34] A.G. Kanaris et al. Optimal design of a plate heat exchanger with undulated surfaces. International Journal of Thermal Sciences, 48:1184-1195, Nov 2008.

[35] H. Wang et al. A simple approach for modelling flow in a microchannel. In Proceedings of SPIE Vol. 4236, Melbourne, Australia, 16 Mar 2001.

[36] T. Zhang et al. Ledinegg instability in microchannels. International Journal of Heat and Mass Transfer, 52:5661-5674, Sept 2009.

[37] J. McLellan et al. Application of kriging-based optimization and smoothed particle hydrodynamics in development of a microchannel heat exchanger model. In $3^{\text {rd }}$ International Technical Meeting on Small Reactors (ITMSR-3), Ottawa, ON, Canada, 5-7 Nov 2014.

[38] Goldak Technologies Inc ${ }^{\circledR}$. Vr software suite highlights. Online Document, 6 Dec 2012. [Cited 8 Jan 2015]. Available: http://www, goldaktec.com.

[39] F.M. White. Viscous Fluid Flow. McGraw-Hill, New York, NY, United States of America, $3^{\text {rd }}$ edition, 2006.

[40] A. Zakharchenko. Development of a holistic model of the carleton supercritical water loop (SCWL). Master of applied science - mechanical engieering, Carleton University, Ottawa, ON, Canada, 2012.

[41] T.L. Bergman et al. Fundamentals of Heat and Mass Transfer. John Wiley \& Sons, Hoboken, NJ, United States of America, $7^{\text {th }}$ edition, 2011.

[42] Jacquet Metal Service. Alloy 600 data sheet. Online Document, 17 Jan 2012. [cited 5 Dec 2014]. Available: http://www.jacquet.biz/JACQUET/USA/files/ JCQusa-alloy-600.pdf.

[43] AZO Materials. Super alloy inconel 690 ${ }^{\mathrm{TM}}$ (uns n06690). Online Document, 11 Jun 2013. [cited 5 Dec 2014]. Available: http://www . azom. com/article .aspx? ArticleID=7747\#4.

[44] National Institute of Standards and Technology. Thermophysical properties of fluid systems. Online Document, 23 Sept 2013. [cited 28 Aug 2014]. Available: http://webbook.nist.gov/chemistry/fluid/. 
[45] B. Mohajer. Convection heat transfer in microchannels. Online Document, 2015. [Cited 22 Feb 2015]. Available: http://www.academia.edu/2006018/ Convection_Heat_Transfer_in_microchannels.

[46] K. Kazemi and J. Goldak. SPH - numerical modeling. Draft Document, 16 Oct 2013.

[47] J. Belin. SPH 2d drop splash with new surface tension model and 2d shading. Video, 2 Aug 2013. [cited 28 Aug 2014]. Available: http://youtu.be/ Daxzw1j7kgU.

[48] T.W. Simpson et al. On the use of statistics in design and the implications for deterministic computer experiments. In 1997 ASME Design Engineering Technical Conferences, Sacramento, CA, USA, 14-17 Sept 1997.

[49] G.G. Wang and S. Shan. Review of metamodeling techniques in support of engineering design optimization. Journal of Mechanical Design, 4:370-380, May 2006.

[50] S. Jeong et al. Efficient optimization design method using kriging model. Journal of Aircraft, 42:413-420, Mar 2005.

[51] L.M. Rios and N.V. Sahinidis. Derivative-free optimization: a review of algorithms and comparison of software implementations. Journal of Global Optimization, 56:1247-1293, Jul 2013.

[52] G. Schmidt. Numerical modelling of the no-core-melt passive safety feature in the generation iv canadian SCWR. Master of applied science - aerospace engieering, Carleton University, Ottawa, ON, Canada, 2014.

[53] N. Ghazi and J.M. Race. Techno-economic modelling and analysis of $\mathrm{CO}_{2}$ pipelines. Journal of Pipeline Engineering, 12:83-92, Jun 2013.

[54] R.G. Alena. Carleton supercritical water loop: Design-driven structural analysis. Master of applied science, Carleton University, Ottawa, ON, Canada, 2010.

[55] M. Nimrouzi. A methodology for optimal design of the canadian SCWR reactor core. Master of applied science - mechanical and aerospace engieering, Carleton University, Ottawa, ON, Canada, 2014.

[56] K. Kazemi. SPH code verification. Draft Document, 17 Aug 2013. 


\section{Appendix A}

\section{VrSuite Solver Verification}

Verification of all VrSuite solvers is well documented, where comparison of simulated results to exact analytical solutions or experimental data for a variety of basic and complex test problems shows good agreement in all cases examined $[40,52,54,55]$. The examples shown in the following sections serve to augment the available verification test cases.

\section{A.1 FEM Solvers}

A test example for the VrSuite thermal solver is detailed in this section.

\section{A.1.1 VrSuite Thermal Solver}

Verification of the VrSuite thermal solver was shown in Section 4.2 while demonstrating the capabilities of the VrSuite optimization tool. In addition to that test, the same problem of one-dimensional heat conduction into a semi-infinite solid will be exhibited here, using a Robin BC instead of the Neumann BC that was previously used. 


\section{Model Description and Analytical Solution}

The solution domain and material properties used are chosen to match those selected for the test case presented in Section 4.2. That is, a $1 \mathrm{~m}$ long steel bar with square $10 \mathrm{~mm}$ by $10 \mathrm{~mm}$ ends, discretized using 200 equally sized 8-node volume elements. The material properties for the bar include density $\rho=7832 \mathrm{~kg} / \mathrm{m}^{3}$, specific heat capacity $\mathrm{c}_{\mathrm{p}}=434 \mathrm{~J} / \mathrm{kg} \cdot \mathrm{K}$, and thermal conductivity $\mathrm{k}=63.9 \mathrm{~W} / \mathrm{m} \cdot \mathrm{K}$, which are chosen to match those given in a sample test problem available in [41]. The entire domain is initialized at a temperature of $\mathrm{T}_{0}=300 \mathrm{~K}$, and a Robin $\mathrm{BC}$ is applied to one end of the bar using an ambient temperature of $\mathrm{T}_{\infty}=400 \mathrm{~K}$ and convective heat transfer coefficient of $\hbar=400 \mathrm{~W} / \mathrm{m}^{2} \cdot \mathrm{K}$. The remaining surfaces of the bar are set to be adiabatic. The exact solution for this problem is as follows [41]:

$$
\frac{T(x, t)-T_{0}}{\left(T_{\infty}-T_{0}\right)}=\operatorname{erfc}\left(\frac{x}{2 \sqrt{\alpha t}}\right)-\left[\exp \left(\frac{\hbar x}{k}+\frac{\hbar^{2} \alpha t}{k^{2}}\right)\right]\left[\operatorname{erfc}\left(\frac{x}{2 \sqrt{\alpha t}}+\frac{\hbar \sqrt{\alpha t}}{k}\right)\right]
$$

where $\mathrm{x}$ represents an axial location along the bar, $\mathrm{t}$ is time, and $\alpha=\mathrm{k} / \rho \mathrm{c}_{\mathrm{p}}$ is the thermal diffusivity.

\section{Results}

The temperature profile within the bar is examined at 5 arbitrarily chosen times of $\mathrm{t}=50 \mathrm{~s}, 100 \mathrm{~s}, 500 \mathrm{~s}, 1000 \mathrm{~s}$, and $1500 \mathrm{~s}$, and are shown in Figure A.1. Upon examination, it is seen that the expected trend of monotonically increasing domain temperature relative to elapsed time is present. The simulated values for domain temperature also agree very well with the analytical solution given by Equation (A.1) for all elapsed times evaluated, showing a relative difference no more than $0.098 \%$ at any location corresponding to a simulated data point. Of importance is that the 
Temperature Profiles for Semi-Infinite Bar with Robin BC

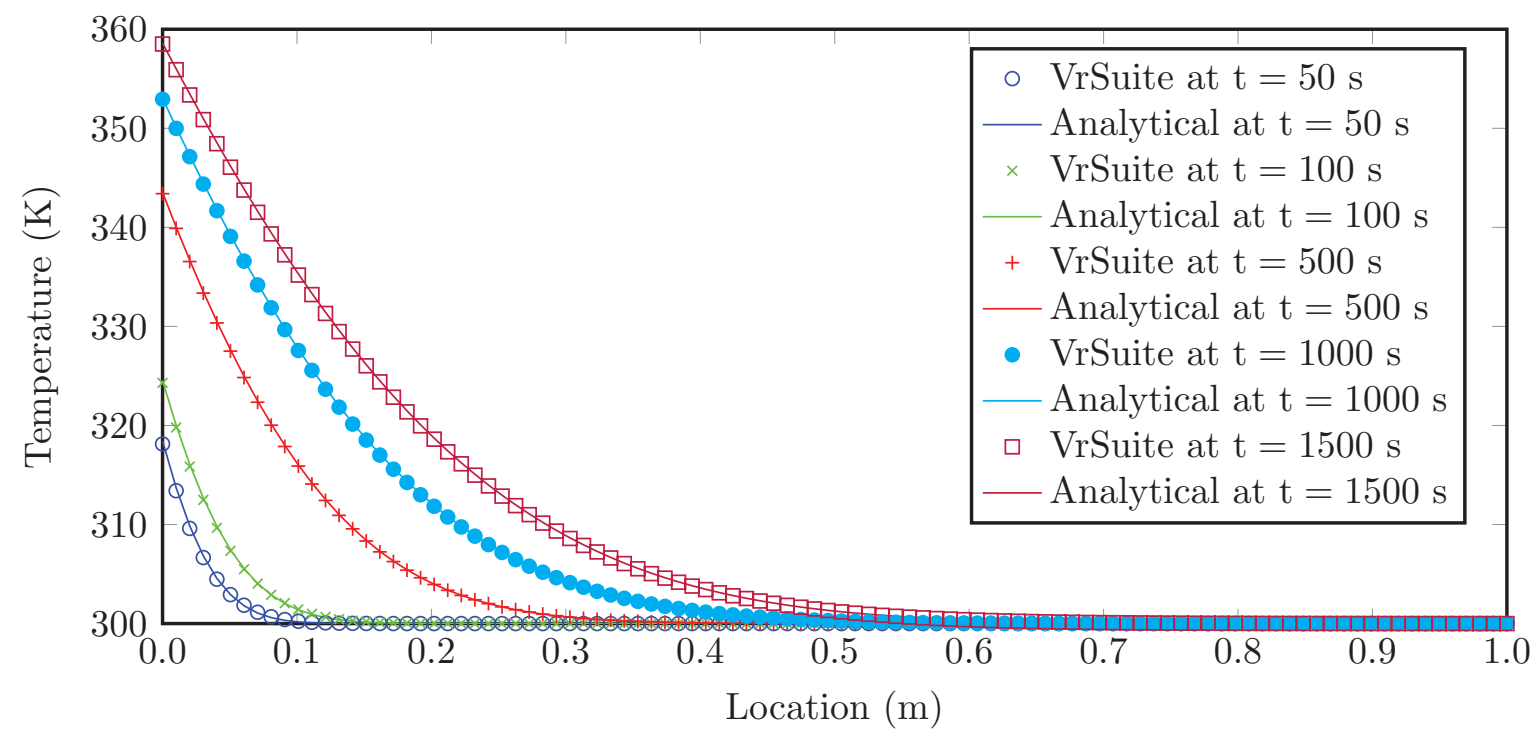

Figure A.1: Temporal evolution of the temperature profile within a semi-infinite bar with 1-D heat conduction and applied Robin BC.

semi-infinite condition is preserved, denoted by a temperature of $\mathrm{T}=300 \mathrm{~K}$ at a location of $\mathrm{x}=1 \mathrm{~m}$ that is equivalent to the initial domain temperature. These results certainly seem to indicate that the VrSuite thermal solver is performing as expected.

\section{A.2 SPH Solvers}

The verification tests performed for the VrSuite SPH solvers, the Navier-Stokes and thermal solvers, are provided in this section.

\section{A.2.1 SPH Thermal Solver}

As with the VrSuite FEM thermal solver, the classical problem of one-dimensional heat conduction into a semi-infinite solid serves as an intuitive option for verification of the SPH thermal solver. A Dirichlet BC will be applied in this example. 


\section{Model Description and Analytical Solution}

For this problem, an identical solution domain to that used in Section 3.4.3 consisting

of a stationary 50 particle by 10 particle array with a spacing of $20 \mathrm{~mm}$ is used. The physical parameters are normalized, with density $\rho=1$, specific heat capacity at constant pressure $\mathrm{c}_{\mathrm{p}}=1$, and thermal conductivity $\mathrm{k}=1$ chosen to match those found in [56] to allow for comparison to their simulated results. The entire domain is subject to the $\mathrm{IC} \mathrm{T}_{0}=0$ and a Dirichlet temperature $\mathrm{BC}$ of $\mathrm{T}_{1}=1$ is applied to one side of the domain throughout the duration of the simulation, while the top and bottom surfaces are adiabatic. The exact solution for this problem is shown below [41]:

$$
T(x, t)=\left(T_{0}-T_{1}\right) \operatorname{erf}\left(\frac{x}{2 \sqrt{\alpha t}}\right)+T_{1}
$$

where $\mathrm{x}$ once again is an axial location along the bar, $\mathrm{t}$ is time, and $\alpha=\mathrm{k} / \rho \mathrm{c}_{\mathrm{p}}$ is the thermal diffusivity.

\section{Results}

Visualizations of the domain temperature profile along the length of the bar at three different instances in time are depicted in Figure A.2. As with the other test cases, the expected general trend of monotonically increasing domain temperature as time progresses is exhibited. The three profiles presented (data set 1) match the analytical solution given by Equation (A.2) very closely, yielding percent differences no greater than $9.40 \%$ at any of the simulated data points. A second set of independently performed analyses (data set 2) also compare favourably with both data set 1 and the analytical solutions, where the small differences between common data points are likely due to slightly different values used for the solver parameters governing convergence and stability. The discrepancies seen between the analytical and simulated 
Temperature Profiles for Semi-Infinite Bar with Dirichlet BC

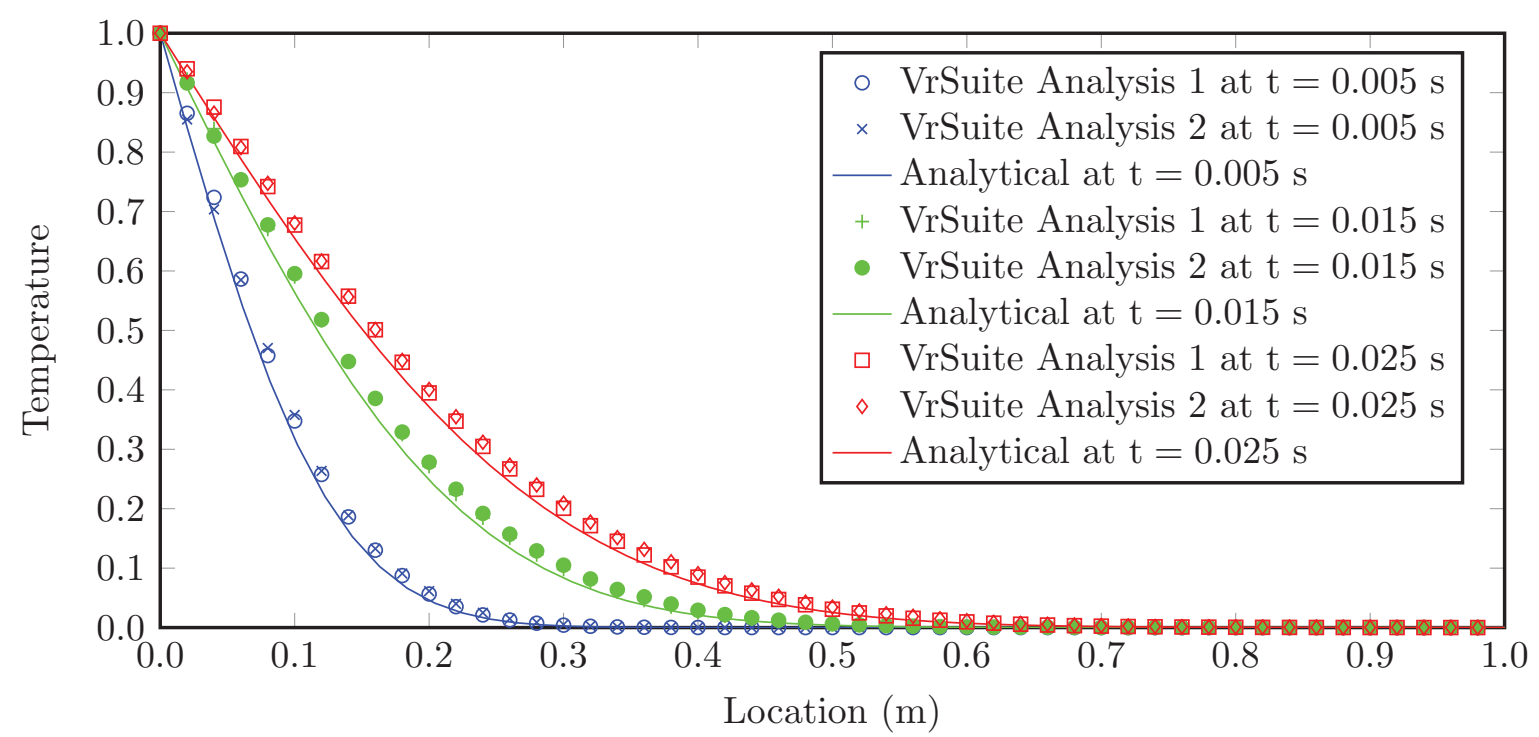

Figure A.2: SPH solver-generated domain temperature profiles in a semi-infinite bar with 1-D heat conduction using a Dirichlet BC.

results are most likely dominated by the coarse distribution of SPH particles, which can easily be improved upon by increasing the number of particles and reducing the particle spacing. Additionally, it is possible to conclude that the semi-infinite condition is preserved given that no end effects are observable at the side of the bar opposite the imposed $\mathrm{BC}$, indicated by temperatures equal to the initial temperature.

\section{A.2.2 Navier-Stokes (NS) Solver}

At the time of writing, it was not possible to perform a thorough evaluation of the VrSuite SPH-NS solver. Required software tools, such as the ability to export fluid velocity profiles at a specified location are not yet available, but are planned for implementation at a later date. Once said functionality is introduced, it is strongly recommended that a thorough verification of the solver be performed. 


\section{Appendix B}

\section{MCHX Optimization: VrSuite-Generated Report}

The optimization report automatically generated by VrSuite for the initial optimization of an MCHX using the $\epsilon$-NTU method detailed in Section 4.3 of Chapter 4 can be found on Page 99. While functional, the software is still in development and the formatting of the report has not yet been finalized. Some items may appear in different locations or will be formatted differently when the report setup is completed.

Note that VrSuite automatically assigns an each design variable an arbitrarily numbered name, starting with X1, when producing plots of the relationship between the objective function and each variable. Table B.1 summarizes these designations. 
Table B.1: VrSuite variable designations for the graphs produced in the automatically generated optimization report.

\begin{tabular}{|c|c|}
\hline Designation & Design Variable \\
\hline \hline X1 & Channel Length, $L$ \\
\hline X2 & Channel Width, $w$ \\
\hline X3 & Channel Wall Thickness, $L_{\text {wall }}$ \\
\hline X4 & Fluid Inlet Velocity, $v$ \\
\hline
\end{tabular}




\section{OPTIMIZATION REPORT}

\section{Contents}

1. Design variable setup 2

2. Block iteration $1 \quad 3$

3. Block iteration $2 \quad 5$

4. Block iteration $3 \quad 7$

5. Block iteration $4 \quad 9$

6. Block iteration 5 11

7. Block iteration $6 \quad 13$

8. $\quad$ Block iteration $7 \quad 15$

9. Block iteration $8 \quad 17$

10. Block iteration $9 \quad 19$

11. Block iteration $10 \quad 21$ 
1. Design variable setup

Project name: Dec12MCHX_OptFn_Simplest

\begin{tabular}{|c|c|c|}
\hline Variable & Min Value & Max Value \\
\hline $\mathrm{L}$ & 0.5 & 15 \\
$\mathrm{w}$ & 0.0005 & 0.075 \\
Lwall & 0.0001 & 0.05 \\
$\mathrm{v}$ & 0.1 & 10 \\
\hline
\end{tabular}

Table 1: Optimization variables setup by user

Block type: Kriging 
2. BLOCK ITERATION 1

\begin{tabular}{|c|c|c|c|c|c|}
\hline Run No. & $\mathrm{L}$ & $\mathrm{w}$ & Lwall & $\mathrm{v}$ & Obj. Value \\
\hline 1 & 2.31488 & 0.0489895 & 0.00844623 & 5.06212 & 4.92521 \\
2 & 4.95078 & 0.0745637 & 0.0220369 & 3.24478 & 8.18823 \\
3 & 1.4496 & 0.0128478 & 0.027603 & 6.4609 & 3.43873 \\
4 & 14.7221 & 0.0370484 & 0.0318078 & 1.13975 & 4.07545 \\
5 & 11.2047 & 0.0590911 & 0.00171404 & 7.90574 & 5.4243 \\
6 & 11.6392 & 0.013326 & 0.0137215 & 9.43106 & 2.9783 \\
7 & 9.0093 & 0.0388965 & 0.00502086 & 2.37326 & 3.90844 \\
8 & 3.26474 & 0.0555545 & 0.0433577 & 4.69747 & 5.79364 \\
9 & 8.17369 & 0.023297 & 0.0459458 & 0.430908 & 0.39268 \\
10 & 12.825 & 0.0679009 & 0.0374066 & 6.96707 & 6.36938 \\
11 & 7.42761 & 0.0274446 & 0.0415867 & 8.67754 & 1.12038 \\
12 & 5.7925 & 0.00397907 & 0.018662 & 3.56234 & 11.0545 \\
\hline Runs: 12 & & \multicolumn{3}{|l}{} & Not completed \\
\hline
\end{tabular}

Table 2: Design variables and objective values for block iteration 1

Number of runs in block: 12

Block solving time: $2 \mathrm{~s}$

Total elapsed time: $2 \mathrm{~s}$ 


$$
X 2=0.0233, X 3=0.0459, X 4=0.4 \quad X 1=8.2, X 3=0.0459, X 4=0.4
$$
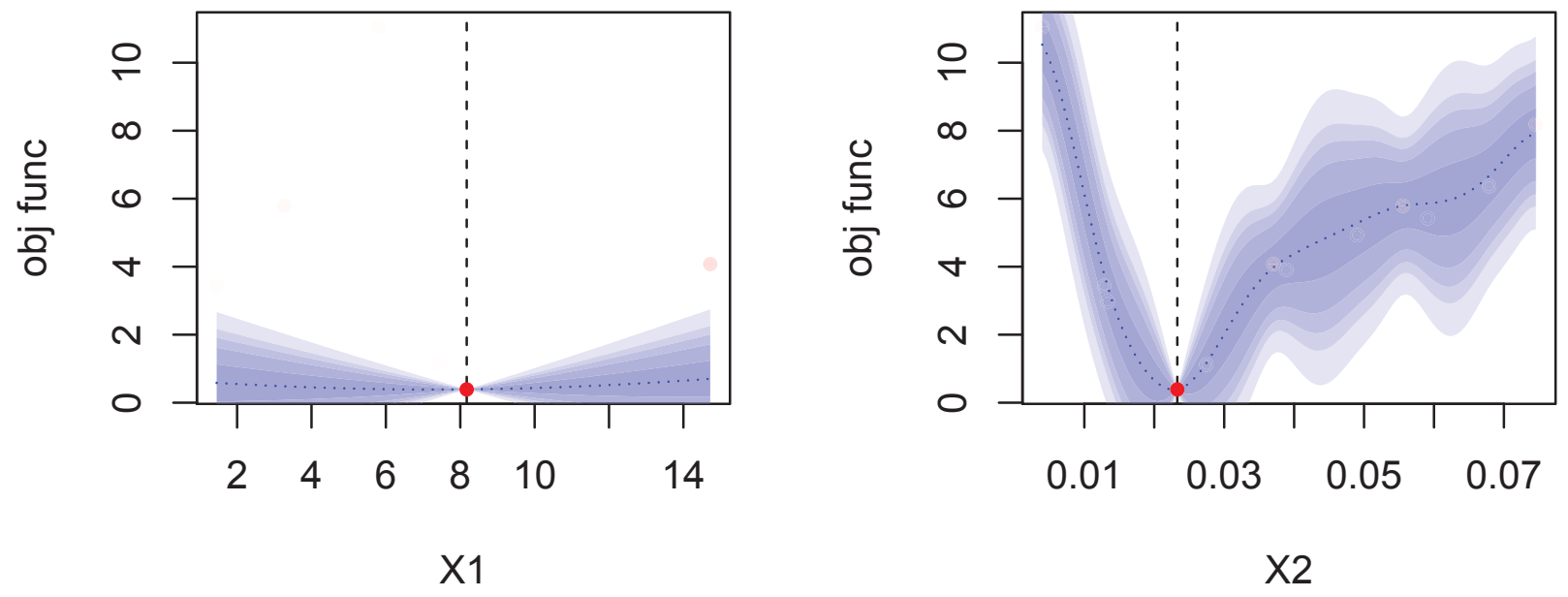

$$
\mathrm{X} 1=8.2, \mathrm{X} 2=0.0233, \mathrm{X} 4=0.4 \quad \mathrm{X} 1=8.2, \mathrm{X} 2=0.0233, \mathrm{X} 3=0.0459
$$

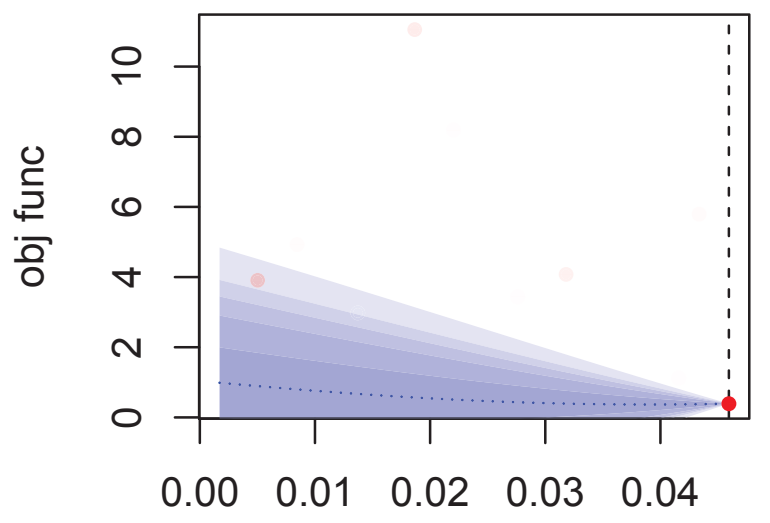

X3

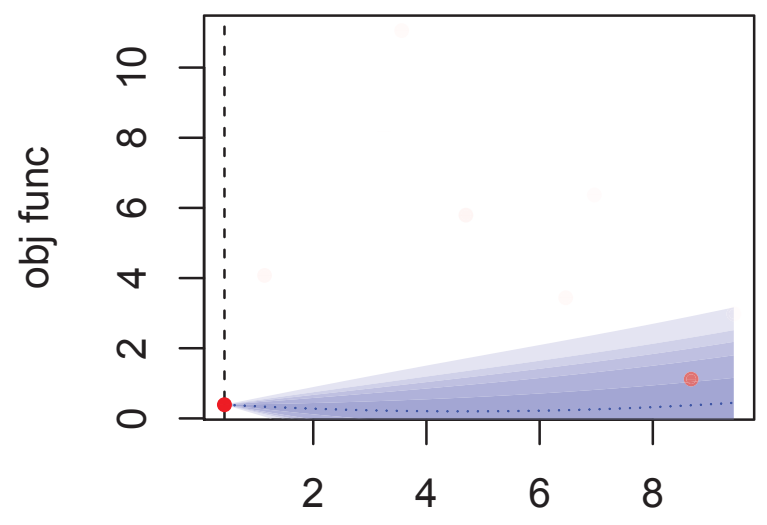

$\mathrm{X} 4$ 
3. BLOCK ITERATION 2

\begin{tabular}{|c|c|c|c|c|c|}
\hline Run No. & $\mathrm{L}$ & $\mathrm{w}$ & Lwall & $\mathrm{v}$ & Obj. Value \\
\hline 13 & 6.56025 & 0.0213426 & 0.0314036 & 7.73487 & 0.402604 \\
14 & 5.65263 & 0.0239003 & 0.00799888 & 2.69928 & 0.442971 \\
15 & 15 & 0.0232547 & 0.0247275 & 4.34829 & 0.230447 \\
16 & 7.883 & 0.0233331 & 0.0294284 & 4.05028 & 0.24797 \\
17 & 11.1624 & 0.0203316 & 0.0106928 & 0.1 & 4.94232 \\
18 & 11.2728 & 0.0214514 & 0.0001 & 8.93914 & 0.363732 \\
19 & 13.1625 & 0.0237766 & 0.000100003 & 0.1 & 3.03742 \\
20 & 12.746 & 0.0198745 & 0.05 & 4.24573 & 0.913618 \\
21 & 10.8013 & 0.0216462 & 0.0115718 & 3.49267 & 0.376192 \\
22 & 11.2223 & 0.0253083 & 0.0001 & 10 & 0.64694 \\
23 & 0.5 & 0.0195903 & 0.05 & 0.1 & 6.33752 \\
24 & 8.21663 & 0.0200656 & 0.0406327 & 0.1 & 5.10546 \\
\hline Runs: 12 & & & & & Not completed \\
\hline
\end{tabular}

Table 3: Design variables and objective values for block iteration 2

Number of runs in block: 12

Block solving time: $1 \mathrm{~s}$

Total elapsed time: $12 \mathrm{~s}$ 


$$
X 2=0.0233, X 3=0.0247, X 4=4.3 \quad X 1=15.0, X 3=0.0247, X 4=4.3
$$
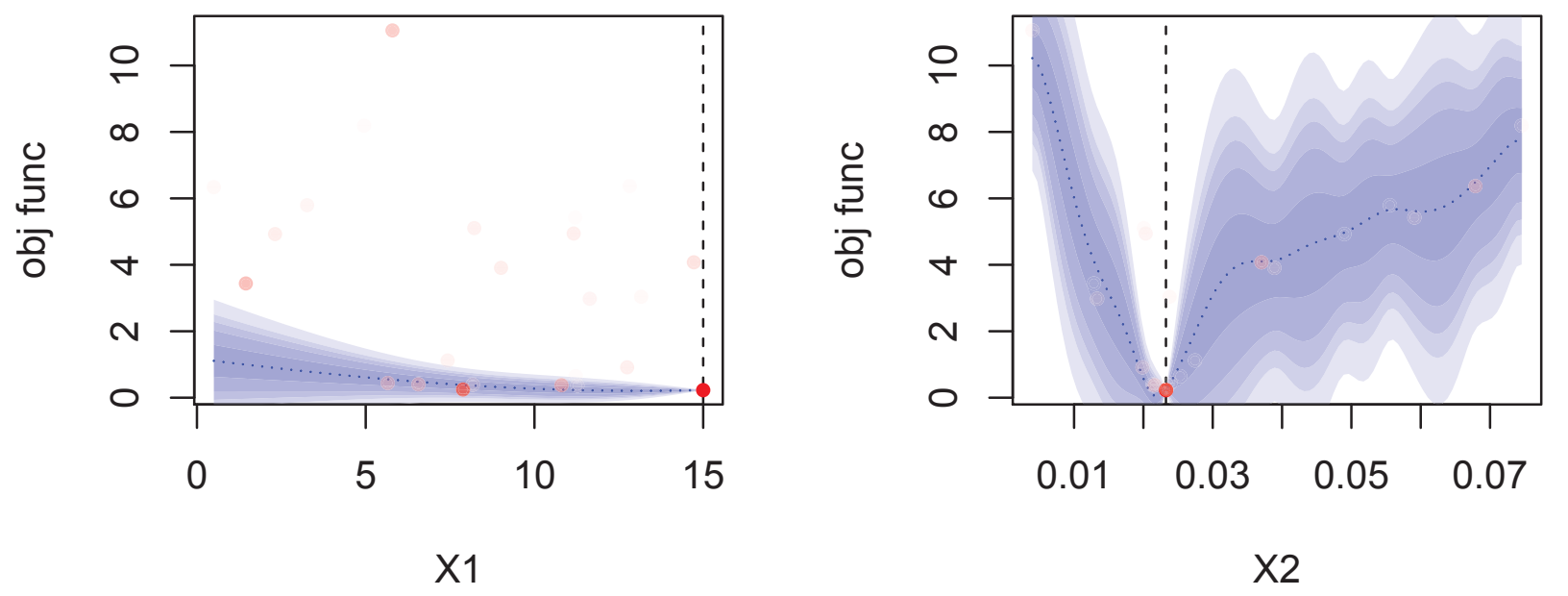

$$
X 1=15.0, X 2=0.0233, X 4=4.3 \quad X 1=15.0, X 2=0.0233, X 3=0.0247
$$
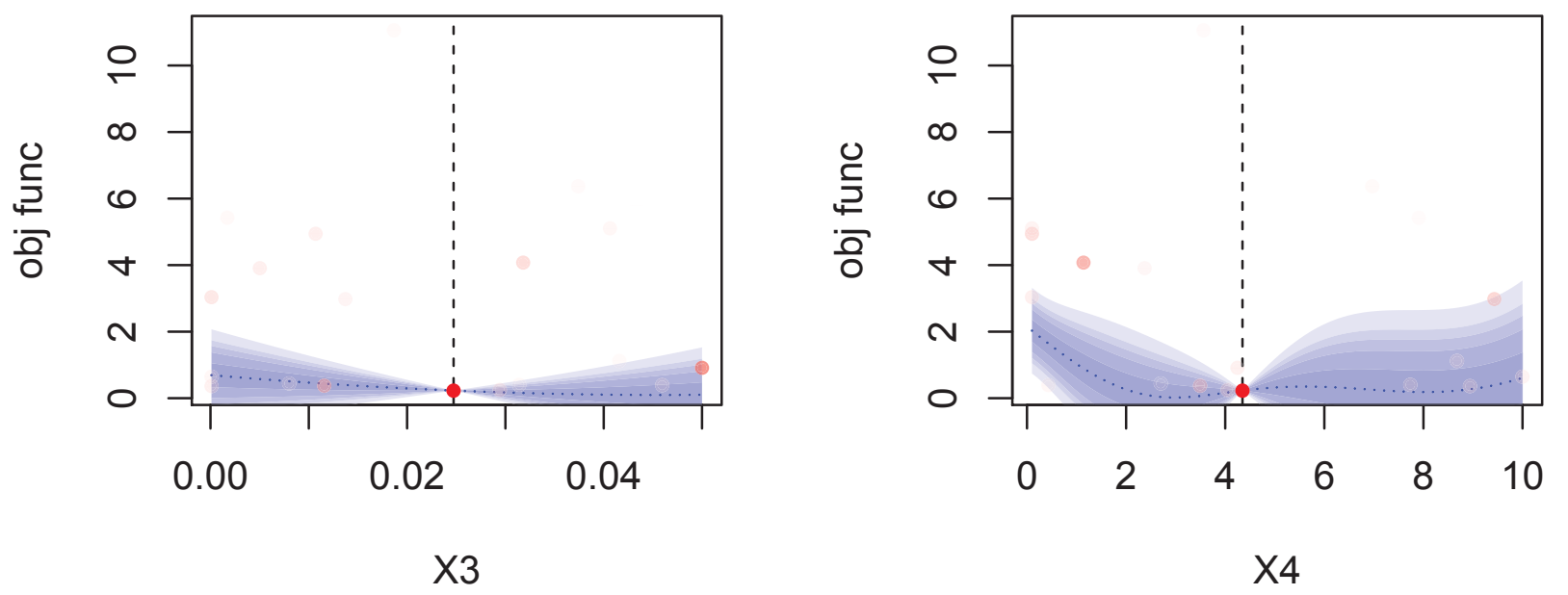
4. Block iteration 3

\begin{tabular}{|c|c|c|c|c|c|}
\hline Run No. & L & w & Lwall & v & Obj. Value \\
\hline 25 & 8.9803 & 0.0241083 & 0.05 & 1.97961 & 0.474242 \\
26 & 15 & 0.0213128 & 0.0155096 & 6.2558 & 0.426569 \\
27 & 15 & 0.0230426 & 0.0387413 & 8.72979 & 0.1858 \\
28 & 0.5 & 0.0248464 & 0.05 & 0.503512 & 0.756437 \\
29 & 9.40382 & 0.023686 & 0.00687756 & 8.063 & 0.317534 \\
30 & 4.75357 & 0.0233914 & 0.0259243 & 9.76923 & 0.254871 \\
31 & 15 & 0.0189377 & 0.0398555 & 8.32755 & 1.07216 \\
32 & 10.1368 & 0.0229036 & 0.0227997 & 8.94908 & 0.157086 \\
33 & 15 & 0.02223 & 0.0452199 & 3.8754 & 0.191891 \\
34 & 11.1101 & 0.0186784 & 0.0001 & 7.36177 & 1.17933 \\
35 & 0.5 & 0.0249849 & 0.0152235 & 8.96887 & 0.59358 \\
36 & 1.13307 & 0.0188815 & 0.00906985 & 9.54388 & 1.06346 \\
\hline Runs: 12 & & \multicolumn{5}{|l|}{} & & Not completed \\
\hline
\end{tabular}

Table 4: Design variables and objective values for block iteration 3

Number of runs in block: 12

Block solving time: $1 \mathrm{~s}$

Total elapsed time: $23 \mathrm{~s}$ 

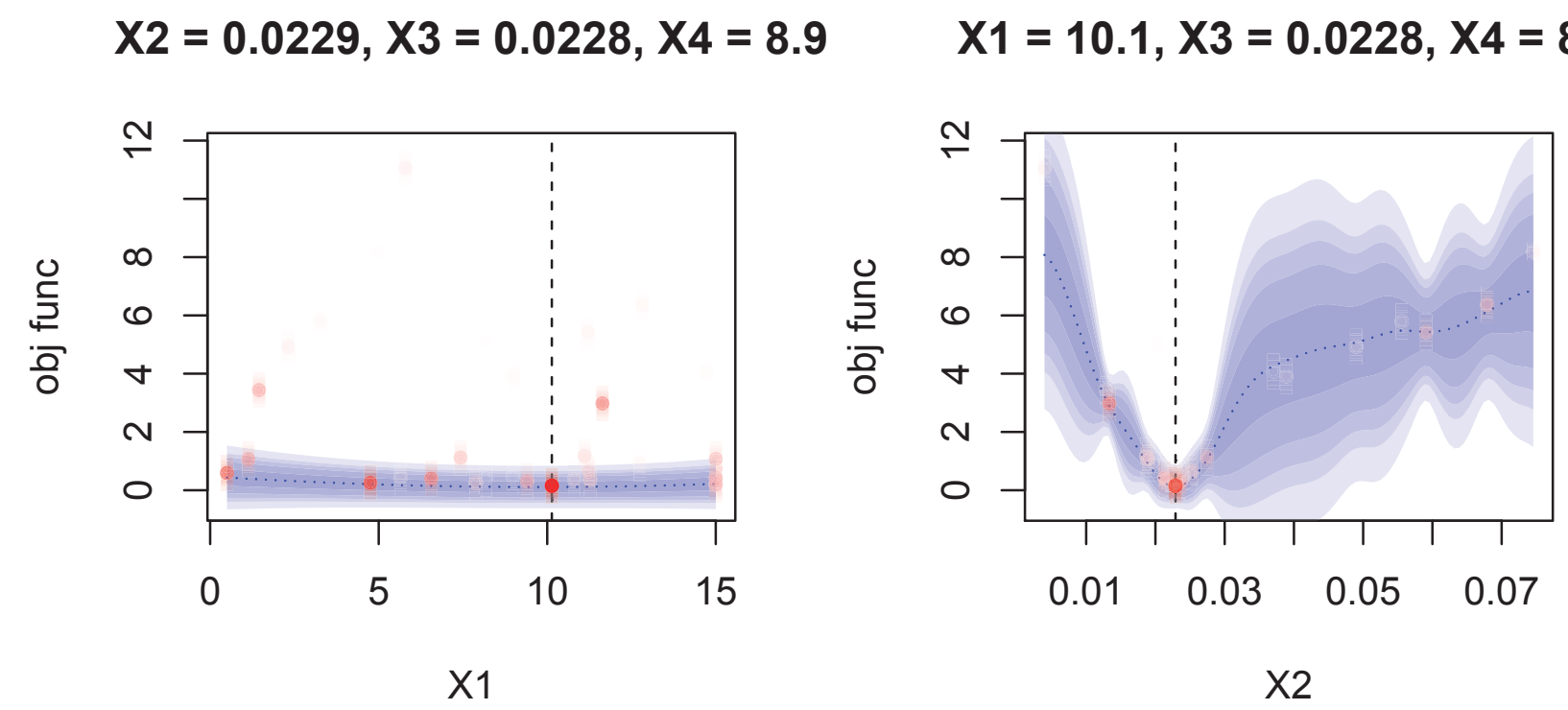

$$
X 1=10.1, X 2=0.0229, X 4=8.9 \quad X 1=10.1, X 2=0.0229, X 3=0.0228
$$
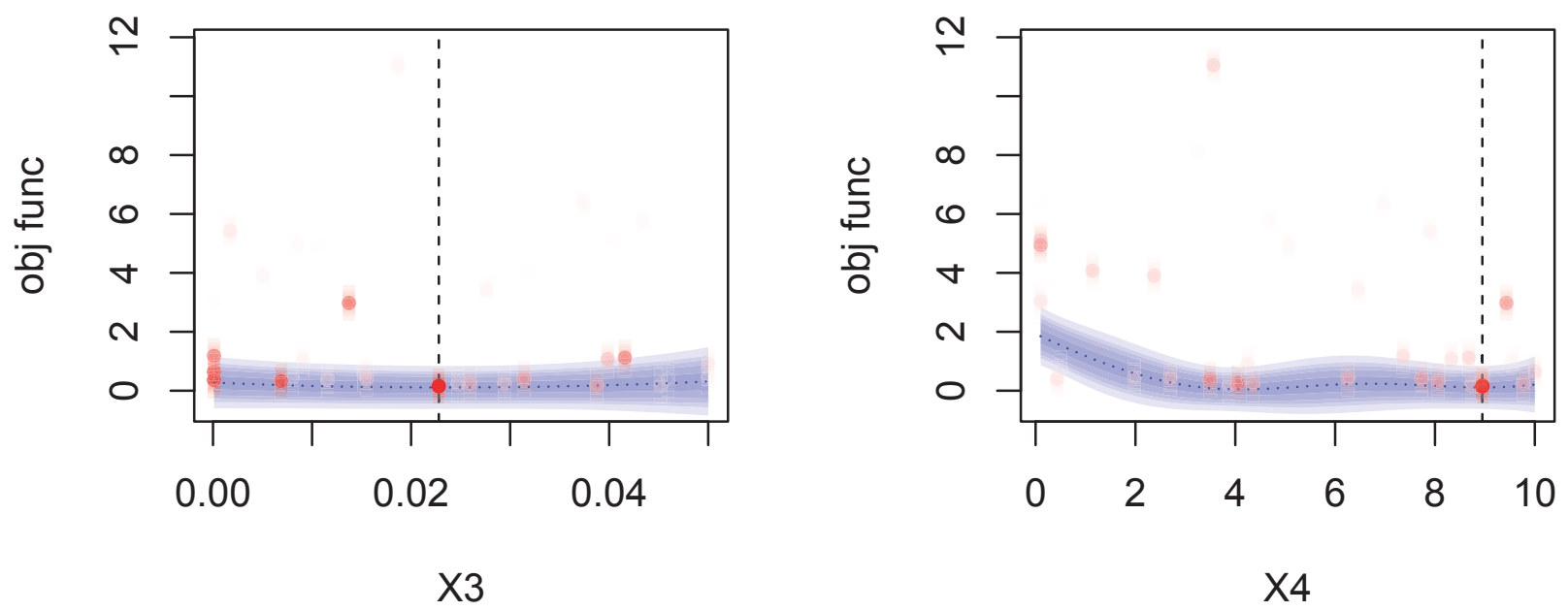
5. BLOCK iteration 4

\begin{tabular}{|c|c|c|c|c|c|}
\hline Run No. & $\mathrm{L}$ & $\mathrm{w}$ & Lwall & $\mathrm{v}$ & Obj. Value \\
\hline 37 & 9.6943 & 0.0225634 & 0.0316133 & 4.20701 & 0.0920682 \\
38 & 10.0434 & 0.022779 & 0.0341742 & 3.70534 & 0.111349 \\
39 & 10.6976 & 0.0224649 & 0.0275081 & 4.428 & 0.118642 \\
40 & 9.97827 & 0.0228657 & 0.0222195 & 9.11909 & 0.149461 \\
41 & 10.3591 & 0.022845 & 0.0355041 & 3.60861 & 0.127135 \\
42 & 9.72235 & 0.0229867 & 0.0224349 & 9.03622 & 0.171012 \\
43 & 11.0115 & 0.0225235 & 0.0293577 & 4.26298 & 0.103204 \\
44 & 10.4619 & 0.0228007 & 0.0339865 & 3.73965 & 0.116924 \\
45 & 11.2564 & 0.0243283 & 0.05 & 9.95526 & 0.434668 \\
46 & 10.571 & 0.0239936 & 0.034332 & 9.32815 & 0.373098 \\
47 & 4.53935 & 0.0225103 & 0.0001 & 5.0587 & 0.102731 \\
48 & 8.49767 & 0.022493 & 0.0148733 & 4.70715 & 0.109723 \\
\hline Runs: 12 & & & & & Not completed \\
\hline
\end{tabular}

Table 5: Design variables and objective values for block iteration 4

Number of runs in block: 12

Block solving time: $2 \mathrm{~s}$

Total elapsed time: $36 \mathrm{~s}$ 


$$
X 2=0.0226, X 3=0.0316, X 4=4.2 \quad X 1=9.7, X 3=0.0316, X 4=4.2
$$
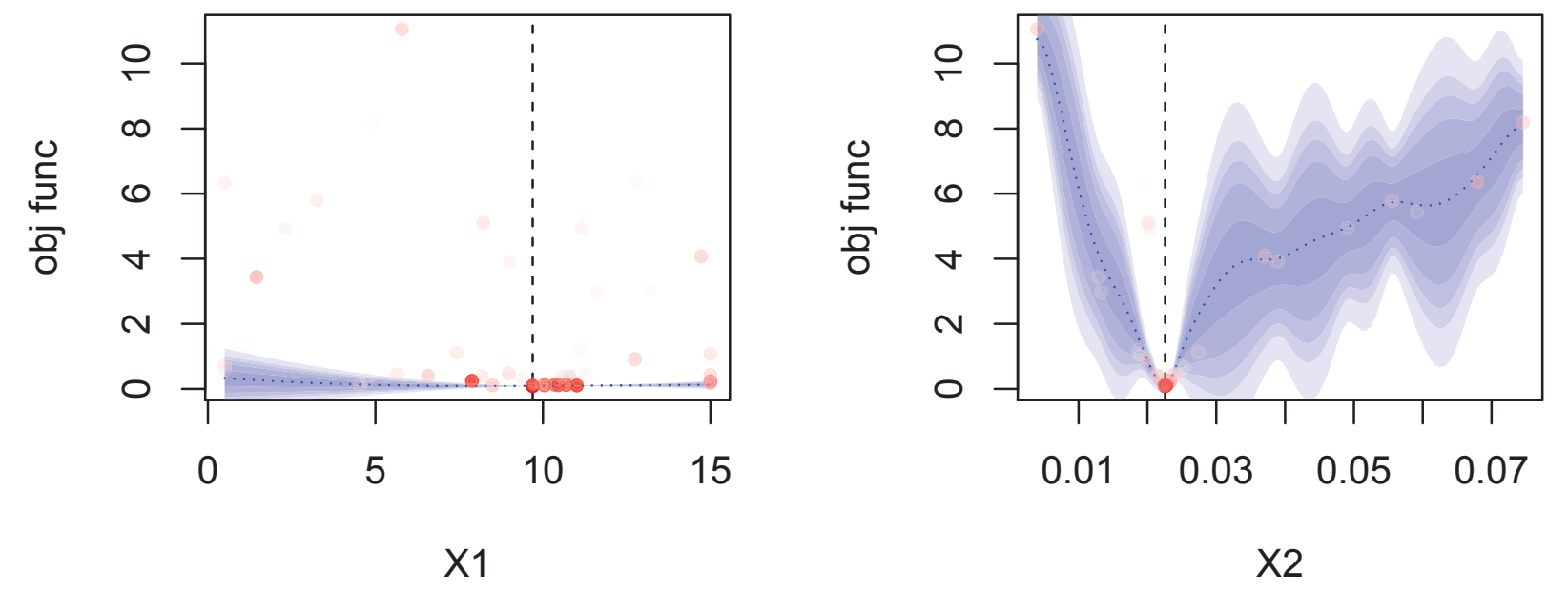

$$
\mathrm{X} 1=9.7, \mathrm{X} 2=0.0226, \mathrm{X} 4=4.2 \quad \mathrm{X} 1=9.7, \mathrm{X} 2=0.0226, \mathrm{X} 3=0.0316
$$
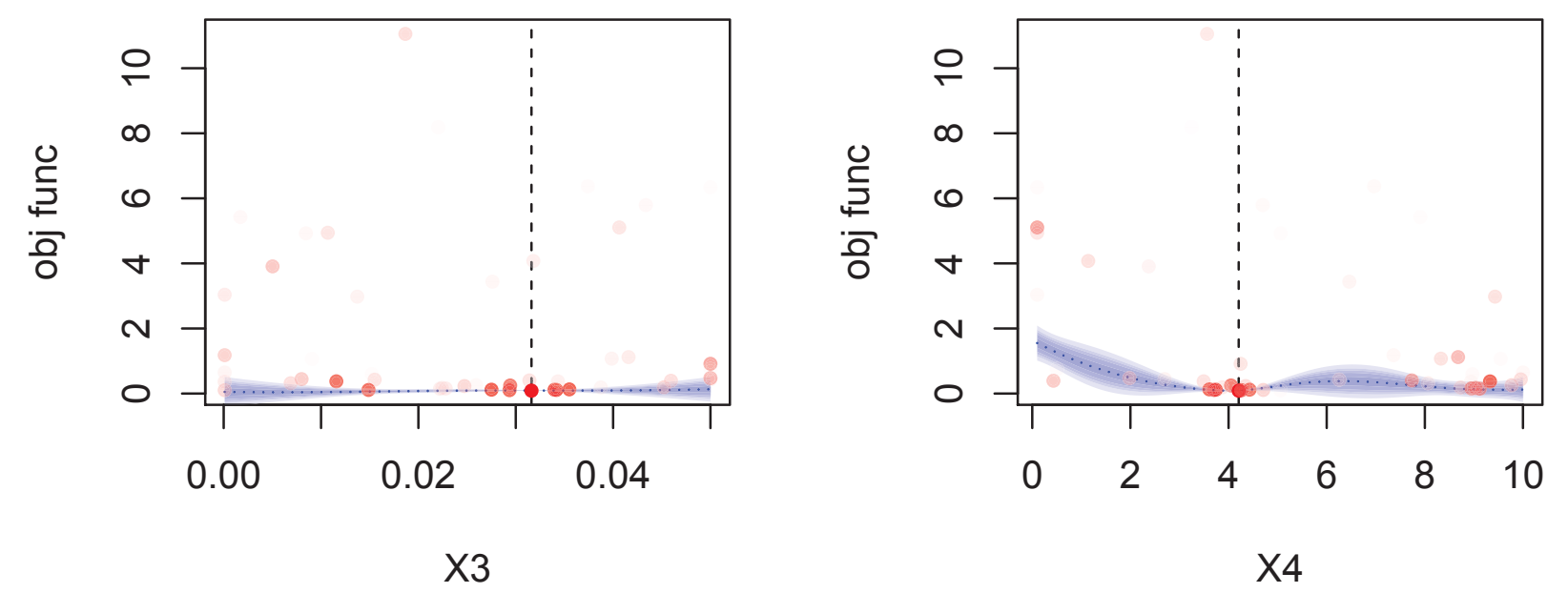
6. BLOCK ITERATION 5

\begin{tabular}{|c|c|c|c|c|c|}
\hline Run No. & $\mathrm{L}$ & $\mathrm{w}$ & Lwall & $\mathrm{v}$ & Obj. Value \\
\hline 49 & 3.28674 & 0.0226133 & 0.0001 & 3.90949 & 0.0814062 \\
50 & 0.5 & 0.0223547 & 0.0001 & 8.91037 & 0.132558 \\
51 & 15 & 0.0268906 & 0.0001 & 6.50593 & 1.05546 \\
52 & 15 & 0.0295885 & 0.0001 & 7.07636 & 1.59711 \\
53 & 15 & 0.028897 & 0.0001 & 5.29803 & 1.54888 \\
54 & 2.74546 & 0.0291074 & 0.0001 & 6.23296 & 1.53664 \\
55 & 7.64051 & 0.0311587 & 0.0001 & 5.75875 & 1.96975 \\
56 & 0.5 & 0.0311807 & 0.0001 & 8.28196 & 1.89671 \\
57 & 5.42037 & 0.0303797 & 0.0001 & 6.99271 & 1.75112 \\
58 & 0.5 & 0.032753 & 0.0001 & 6.72154 & 2.2773 \\
59 & 0.5 & 0.0313214 & 0.0001 & 6.15669 & 2.03719 \\
60 & 13.3745 & 0.0327459 & 0.0001 & 8.31433 & 2.11564 \\
\hline Runs: 12 & & & & & Not completed \\
\hline
\end{tabular}

Table 6: Design variables and objective values for block iteration 5

Number of runs in block: 12

Block solving time: $2 \mathrm{~s}$

Total elapsed time: $47 \mathrm{~s}$ 
$\mathrm{X} 2=0.0226, \mathrm{X} 3=0.0001, \mathrm{X} 4=3.9 \quad \mathrm{X} 1=3.3, \mathrm{X} 3=0.0001, \mathrm{X} 4=3.9$
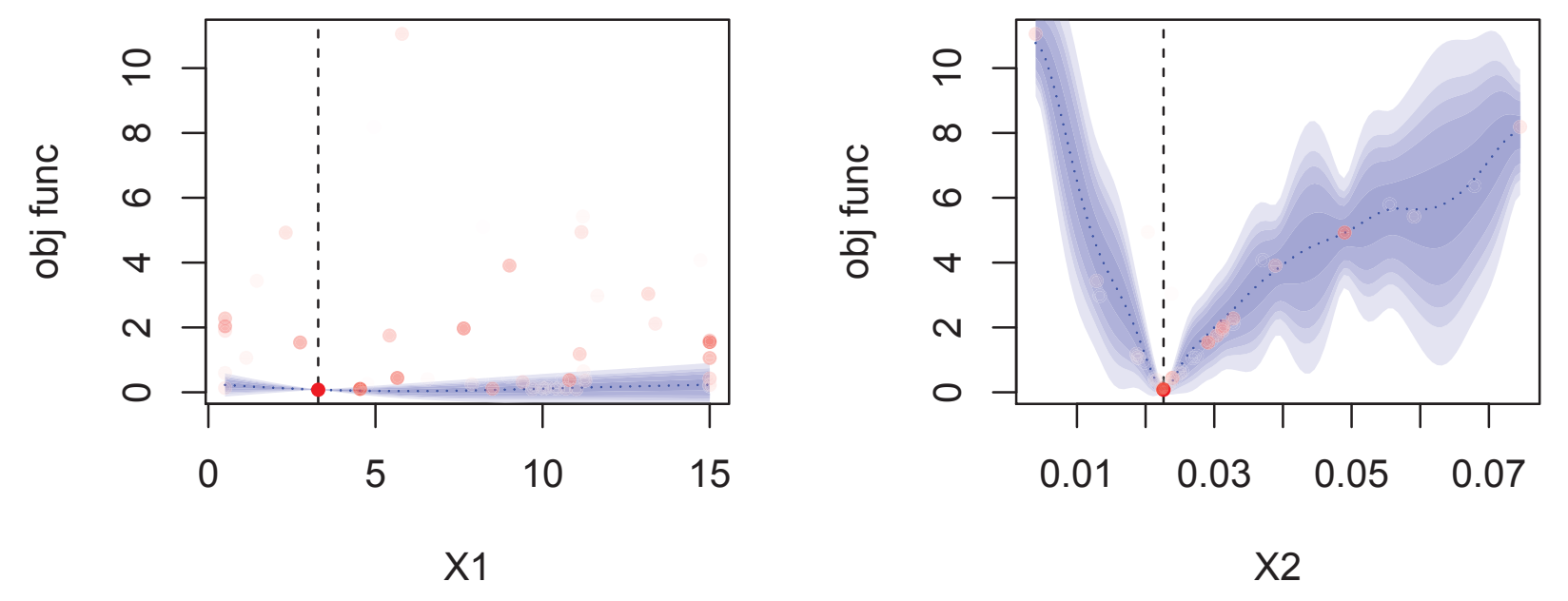

$\mathrm{X} 1=3.3, \mathrm{X} 2=0.0226, \mathrm{X} 4=3.9 \quad \mathrm{X} 1=3.3, \mathrm{X} 2=0.0226, \mathrm{X} 3=0.0001$
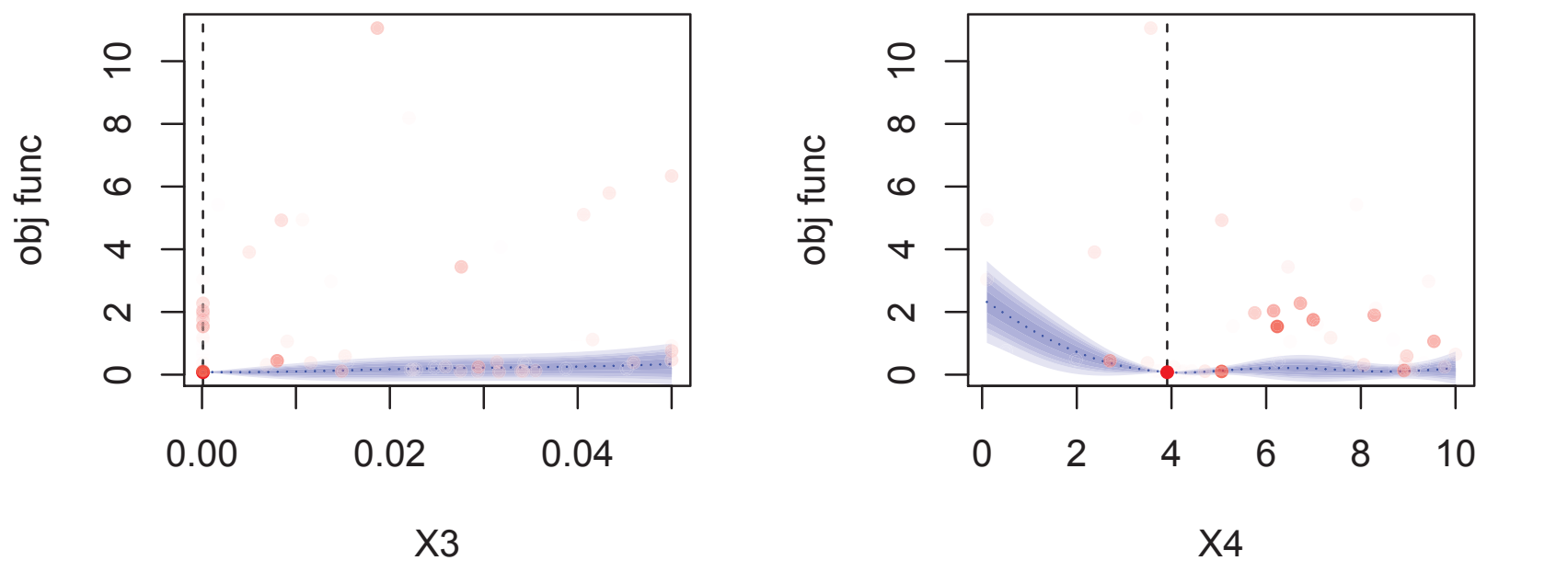
7. BLOCK iteration 6

\begin{tabular}{|c|c|c|c|c|c|}
\hline Run No. & $\mathrm{L}$ & $\mathrm{w}$ & Lwall & $\mathrm{v}$ & Obj. Value \\
\hline 61 & 0.5 & 0.022088 & 0.05 & 10 & 0.208321 \\
62 & 6.46998 & 0.0221589 & 0.05 & 10 & 0.178477 \\
63 & 15 & 0.0225583 & 0.0342268 & 10 & 0.0877012 \\
64 & 6.68902 & 0.0379718 & 0.05 & 10 & 2.84874 \\
65 & 15 & 0.0391401 & 0.05 & 10 & 2.99831 \\
66 & 15 & 0.0372262 & 0.05 & 10 & 2.73093 \\
67 & 12.1892 & 0.038211 & 0.05 & 8.71537 & 2.94849 \\
68 & 12.0506 & 0.0382598 & 0.0288578 & 9.90378 & 2.88311 \\
69 & 7.7889 & 0.0406837 & 0.0394814 & 9.43072 & 3.25037 \\
70 & 10.4182 & 0.0388801 & 0.0446301 & 9.6276 & 2.98618 \\
71 & 15 & 0.0419369 & 0.0307303 & 9.72272 & 3.38496 \\
72 & 15 & 0.0408291 & 0.0364469 & 8.82611 & 3.30243 \\
\hline Runs: 12 & & & & & Not completed \\
\hline
\end{tabular}

Table 7: Design variables and objective values for block iteration 6

Number of runs in block: 12

Block solving time: $2 \mathrm{~s}$

Total elapsed time: $58 \mathrm{~s}$ 
$\mathrm{X} 2=0.0226, \mathrm{X} 3=0.0001, \mathrm{X} 4=3.9 \quad \mathrm{X} 1=3.3, \mathrm{X} 3=0.0001, \mathrm{X} 4=3.9$
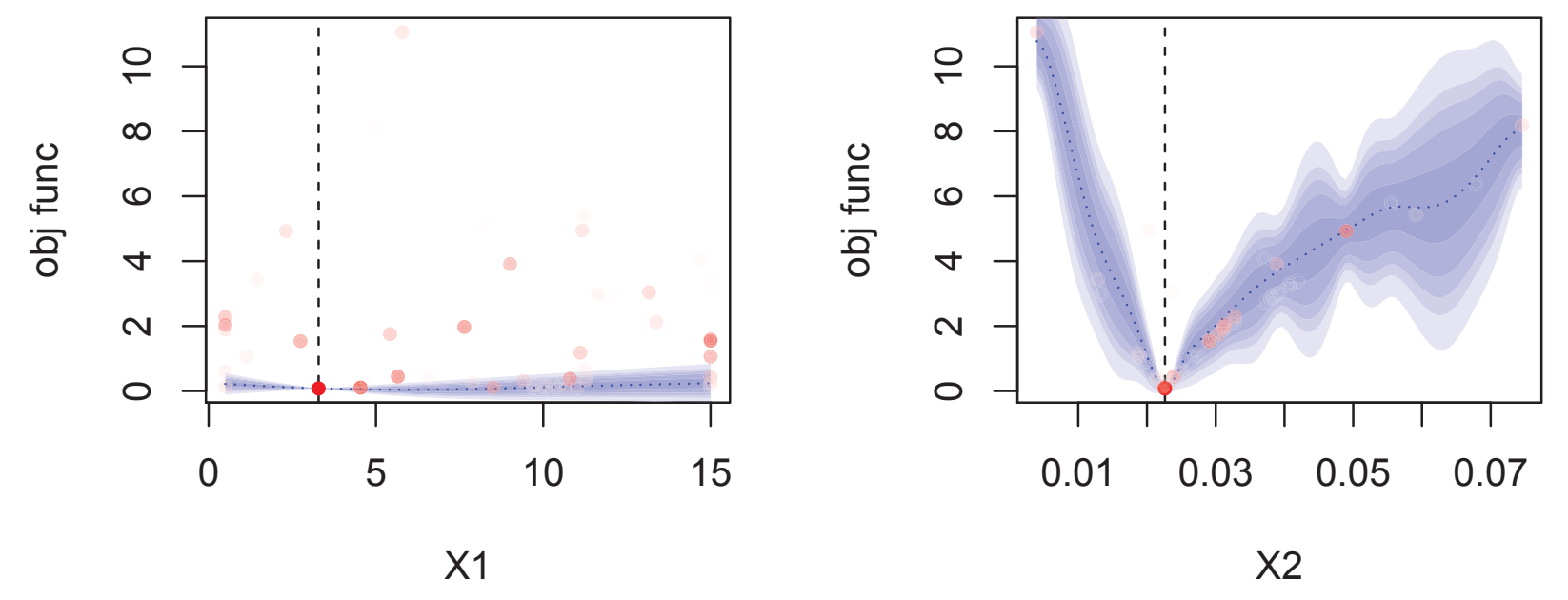

$\mathrm{X} 1=3.3, \mathrm{X} 2=0.0226, \mathrm{X} 4=3.9 \quad \mathrm{X} 1=3.3, \mathrm{X} 2=0.0226, \mathrm{X} 3=0.0001$
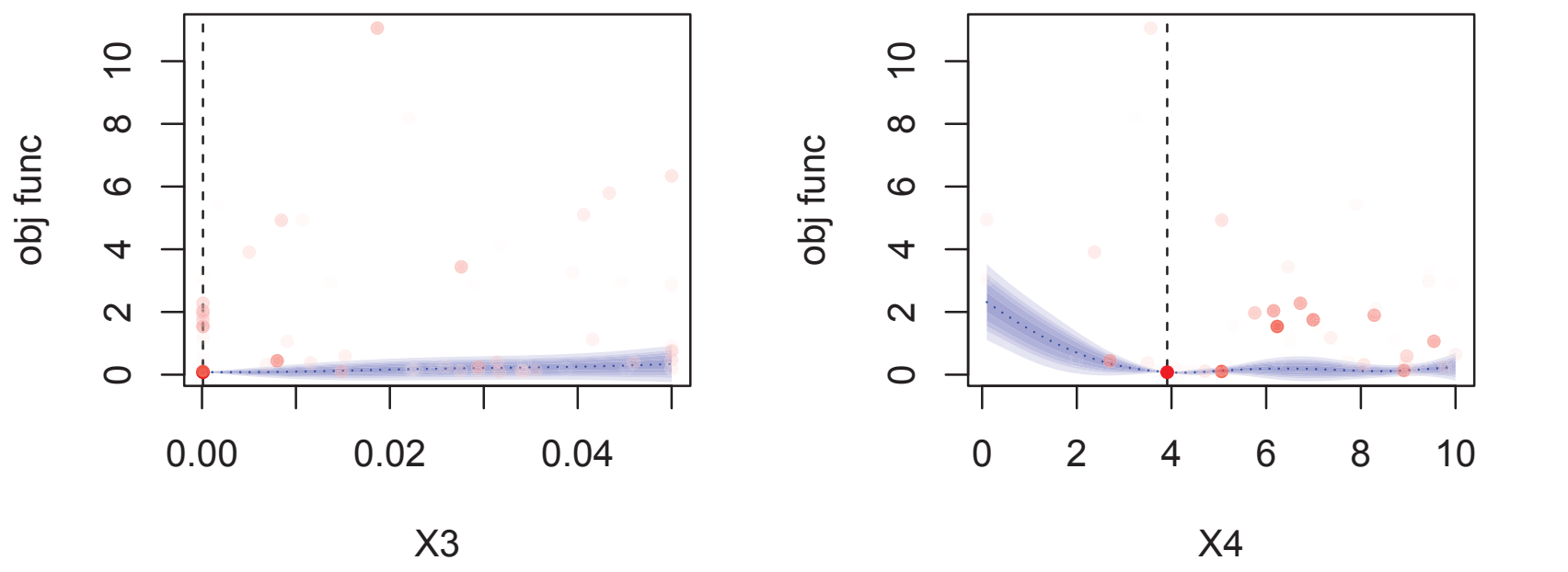
8. BLOCK ITERATION 7

\begin{tabular}{|c|c|c|c|c|c|}
\hline Run No. & $\mathrm{L}$ & $\mathrm{w}$ & Lwall & $\mathrm{v}$ & Obj. Value \\
\hline 73 & 5.26643 & 0.0232907 & 0.05 & 1.41052 & 0.205457 \\
74 & 15 & 0.0457339 & 0.05 & 0.1 & 8.13829 \\
75 & 15 & 0.0474965 & 0.05 & 0.1 & 8.62836 \\
76 & 15 & 0.0466051 & 0.0229811 & 0.1 & 8.3828 \\
77 & 8.9916 & 0.0465431 & 0.0426583 & 0.1 & 8.36702 \\
78 & 13.9228 & 0.0466015 & 0.0416892 & 0.1 & 8.38397 \\
79 & 15 & 0.0488033 & 0.0001 & 0.1 & 8.97723 \\
80 & 12.2684 & 0.048486 & 0.0176074 & 0.1 & 8.89327 \\
81 & 15 & 0.0500987 & 0.05 & 0.1 & 9.31341 \\
82 & 6.19472 & 0.044524 & 0.05 & 0.1 & 7.8 \\
83 & 9.12849 & 0.0451448 & 0.0001 & 0.1 & 7.97203 \\
84 & 15 & 0.0490427 & 0.043385 & 0.1 & 9.04103 \\
\hline Runs: 12 & & & & & Not completed \\
\hline
\end{tabular}

Table 8: Design variables and objective values for block iteration 7

Number of runs in block: 12

Block solving time: $2 \mathrm{~s}$

Total elapsed time: $70 \mathrm{~s}$ 
$\mathrm{X} 2=0.0226, \mathrm{X} 3=0.0001, \mathrm{X} 4=3.9 \quad \mathrm{X} 1=3.3, \mathrm{X} 3=0.0001, \mathrm{X} 4=3.9$
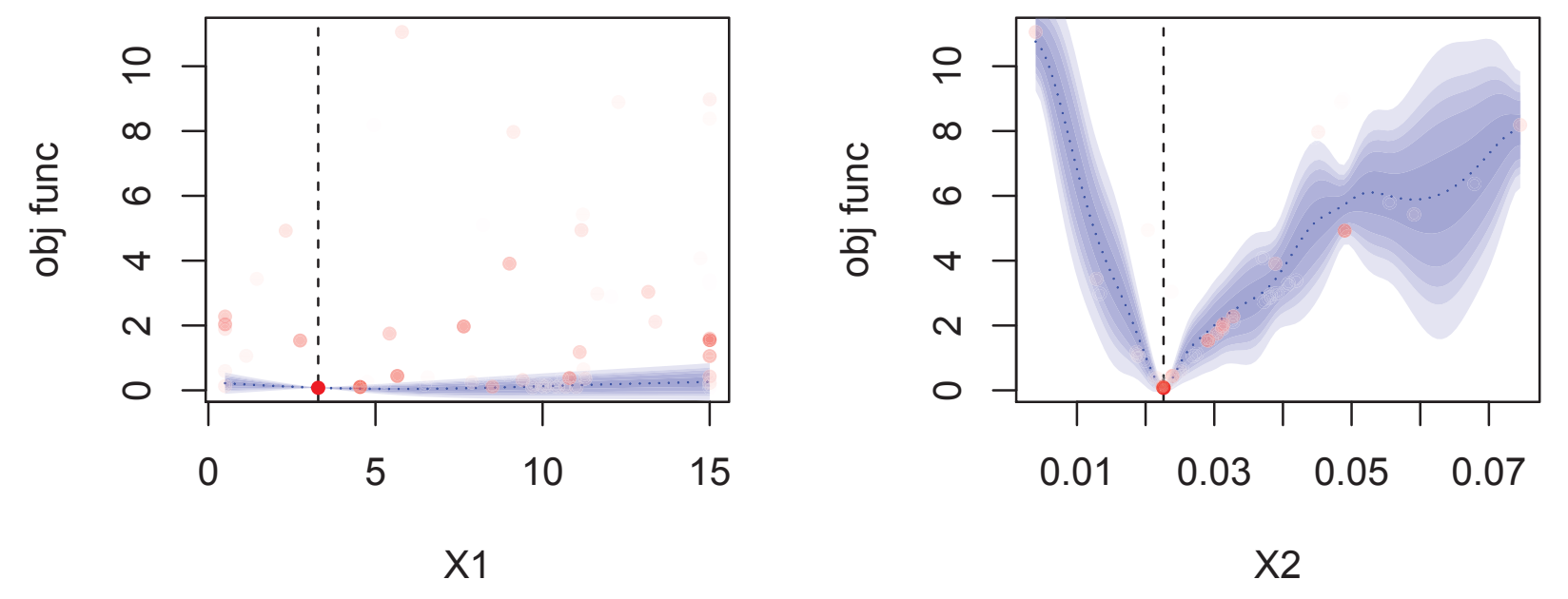

$\mathrm{X} 1=3.3, \mathrm{X} 2=0.0226, \mathrm{X} 4=3.9 \quad \mathrm{X} 1=3.3, \mathrm{X} 2=0.0226, \mathrm{X} 3=0.0001$
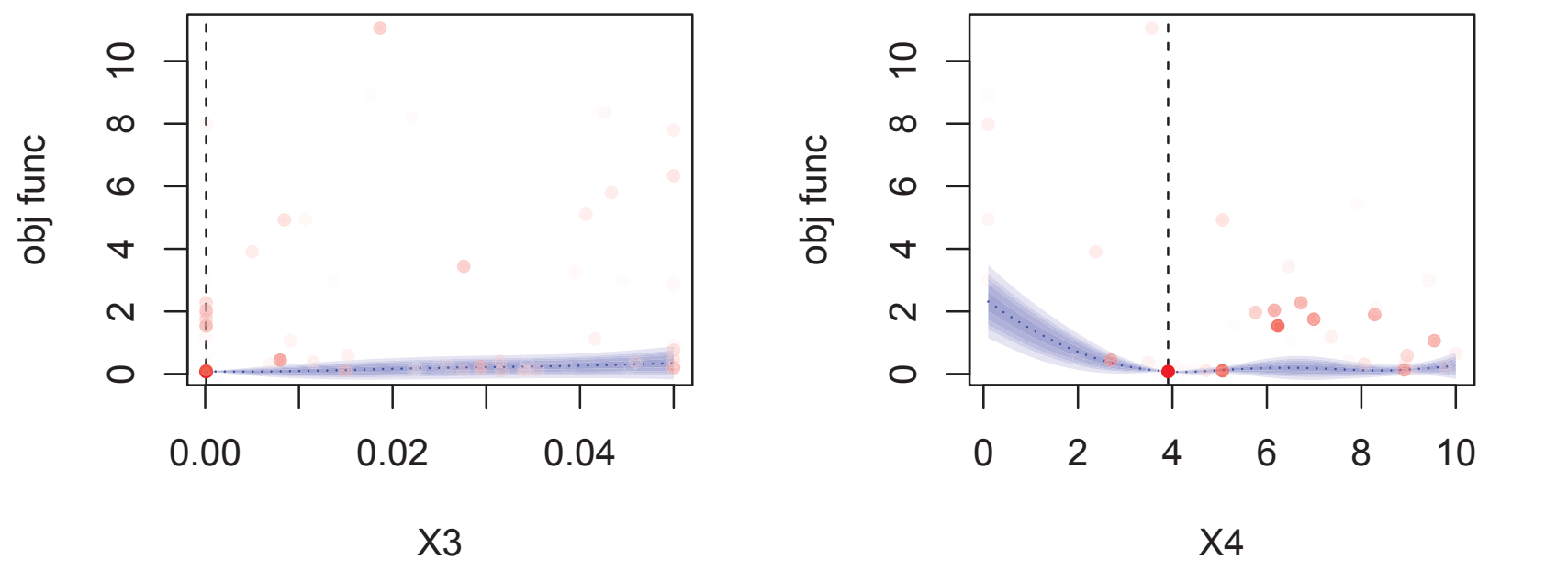
9. BLOCK ITERATION 8

\begin{tabular}{|c|c|c|c|c|c|}
\hline Run No. & L & w & Lwall & v & Obj. Value \\
\hline 85 & 6.85587 & 0.0225843 & 0.0001 & 4.19674 & 0.0848904 \\
86 & 15 & 0.0227736 & 0.00949943 & 8.75634 & 0.130974 \\
87 & 5.27661 & 0.0224905 & 0.00916522 & 4.14887 & 0.113441 \\
88 & 5.62621 & 0.0296645 & 0.05 & 0.1 & 2.39695 \\
89 & 0.993415 & 0.0309519 & 0.05 & 0.1 & 2.97341 \\
90 & 1.45259 & 0.0302898 & 0.0336464 & 0.1 & 2.68203 \\
91 & 0.5 & 0.0226468 & 0.0001 & 6.87603 & 0.10235 \\
92 & 9.0922 & 0.0224268 & 0.05 & 4.09366 & 0.132381 \\
93 & 14.5398 & 0.0226557 & 0.0150017 & 10 & 0.107855 \\
94 & 2.72626 & 0.0302162 & 0.0467501 & 0.1 & 2.64778 \\
95 & 4.36311 & 0.031494 & 0.0363392 & 0.1 & 3.2142 \\
96 & 0.5 & 0.0314459 & 0.0325708 & 0.1 & 3.18786 \\
\hline Runs: 12 & & & & & Not completed \\
\hline
\end{tabular}

Table 9: Design variables and objective values for block iteration 8

Number of runs in block: 12

Block solving time: $2 \mathrm{~s}$

Total elapsed time: $83 \mathrm{~s}$ 
$\mathrm{X} 2=0.0226, \mathrm{X} 3=0.0001, \mathrm{X} 4=3.9 \quad \mathrm{X} 1=3.3, \mathrm{X} 3=0.0001, \mathrm{X} 4=3.9$
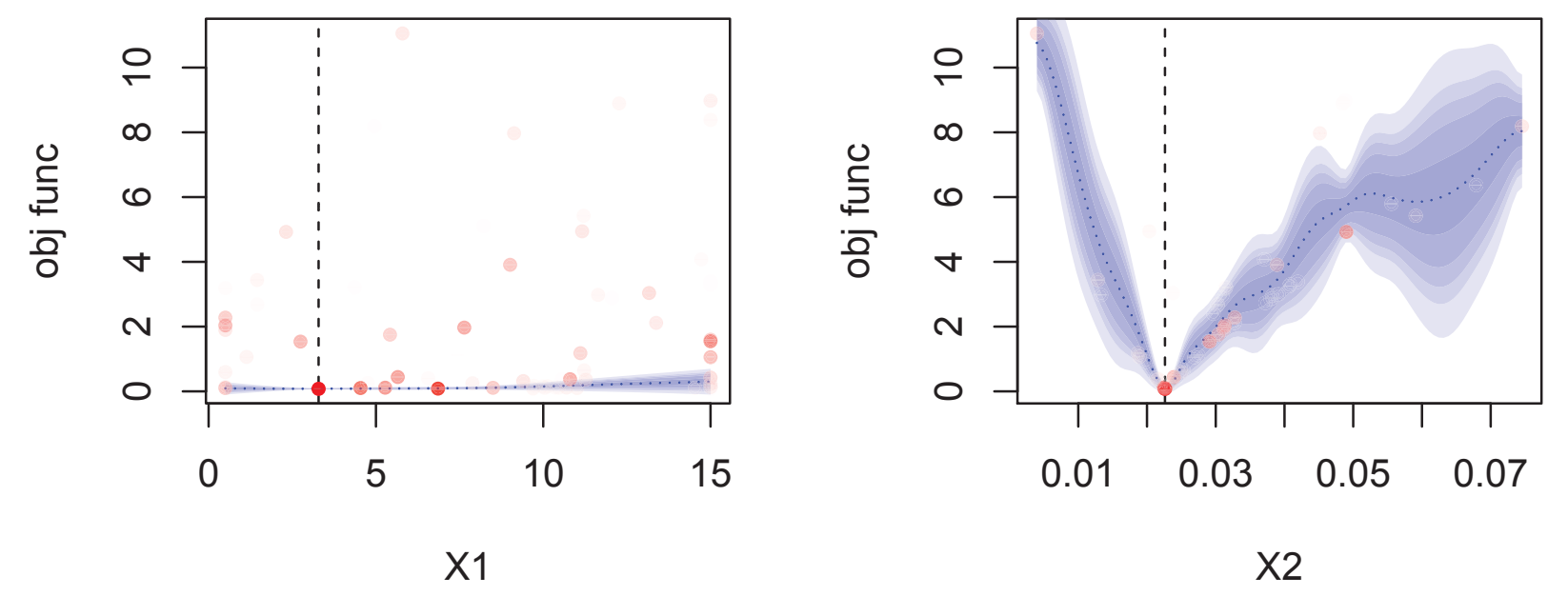

$\mathrm{X} 1=3.3, \mathrm{X} 2=0.0226, \mathrm{X} 4=3.9 \quad \mathrm{X} 1=3.3, \mathrm{X} 2=0.0226, \mathrm{X} 3=0.0001$
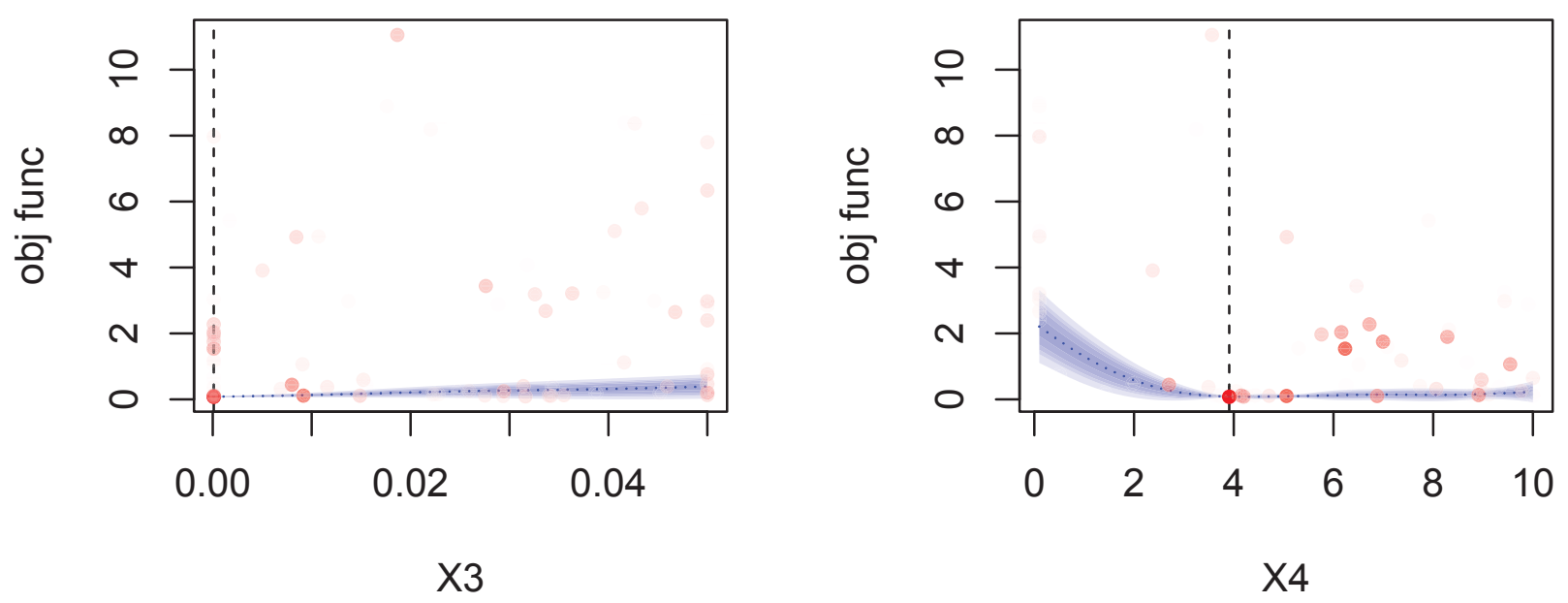
10. BLOCK ITERATION 9

\begin{tabular}{|c|c|c|c|c|c|}
\hline Run No. & $\mathrm{L}$ & $\mathrm{w}$ & Lwall & $\mathrm{v}$ & Obj. Value \\
\hline 97 & 0.5 & 0.0225652 & 0.05 & 8.43636 & 0.0842688 \\
98 & 0.5 & 0.0228759 & 0.0001 & 4.63985 & 0.144062 \\
99 & 0.5 & 0.0227752 & 0.0001 & 8.0096 & 0.130124 \\
100 & 0.5 & 0.0223493 & 0.05 & 6.21252 & 0.152587 \\
101 & 8.74224 & 0.0223599 & 0.05 & 5.82349 & 0.141029 \\
102 & 0.5 & 0.0227775 & 0.0001 & 5.6867 & 0.123803 \\
103 & 0.5 & 0.0224965 & 0.05 & 7.27882 & 0.107727 \\
104 & 0.5 & 0.0225247 & 0.0374445 & 8.42183 & 0.0976556 \\
105 & 13.0778 & 0.0225654 & 0.05 & 9.84864 & 0.0897764 \\
106 & 0.5 & 0.022849 & 0.0001 & 3.67118 & 0.127987 \\
107 & 0.5 & 0.0636752 & 0.0001 & 0.1 & 12.9766 \\
108 & 8.39217 & 0.0639485 & 0.0001 & 0.1 & 12.4523 \\
\hline Runs: 12 & & & & & Not completed \\
\hline
\end{tabular}

Table 10: Design variables and objective values for block iteration 9

Number of runs in block: 12

Block solving time: $3 \mathrm{~s}$

Total elapsed time: $96 \mathrm{~s}$ 
$\mathrm{X} 2=0.0226, \mathrm{X} 3=0.0001, \mathrm{X} 4=3.9 \quad \mathrm{X} 1=3.3, \mathrm{X} 3=0.0001, \mathrm{X} 4=3.9$
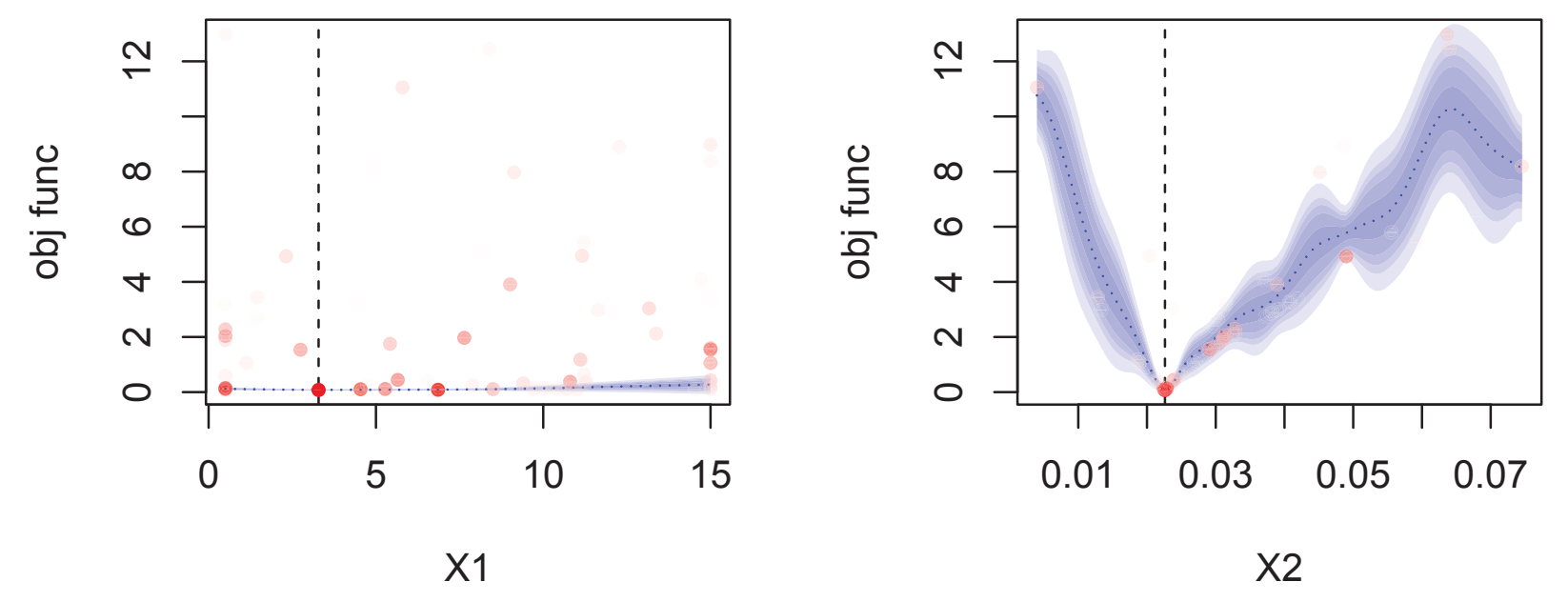

$\mathrm{X} 1=3.3, \mathrm{X} 2=0.0226, \mathrm{X} 4=3.9 \quad \mathrm{X} 1=3.3, \mathrm{X} 2=0.0226, \mathrm{X} 3=0.0001$
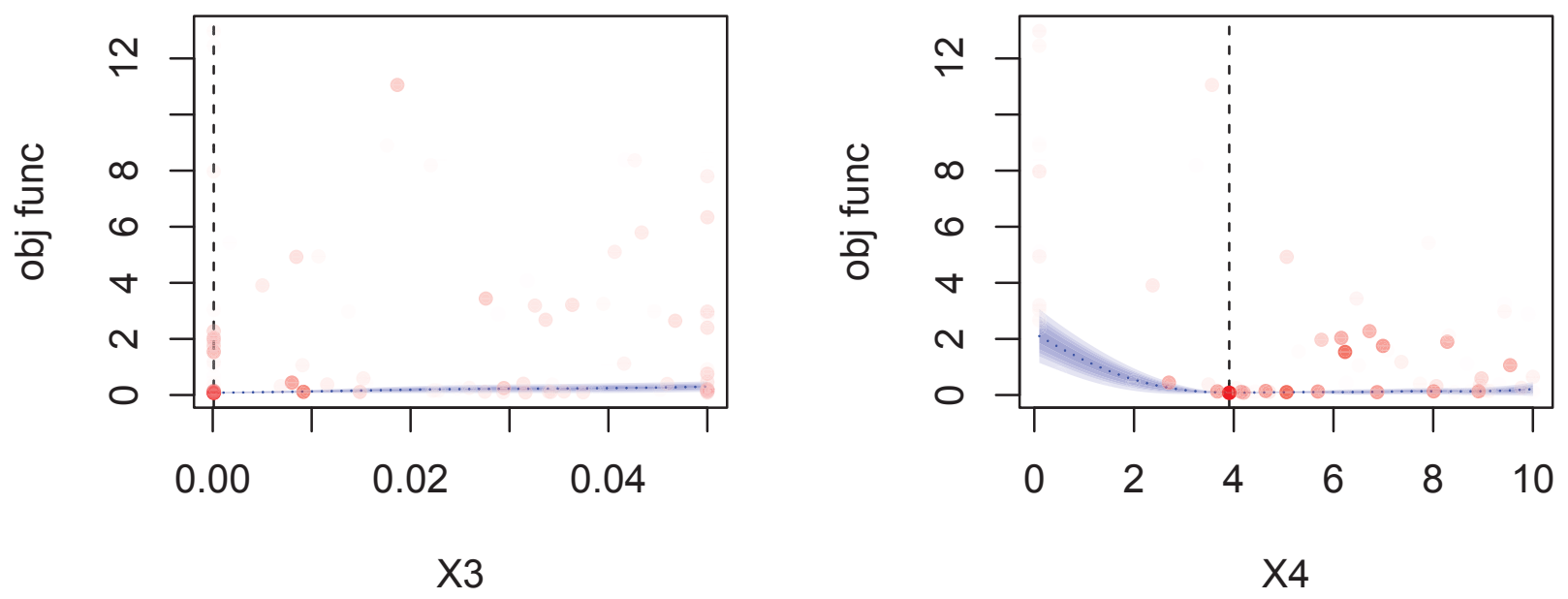
11. Block iteration 10

\begin{tabular}{|c|c|c|c|c|c|}
\hline Run No. & $\mathrm{L}$ & $\mathrm{w}$ & Lwall & $\mathrm{v}$ & Obj. Value \\
\hline 109 & 0.5 & 0.0518842 & 0.05 & 10 & 5.18247 \\
110 & 8.30762 & 0.0518782 & 0.05 & 10 & 4.54162 \\
111 & 4.3763 & 0.0522913 & 0.0300993 & 10 & 4.63768 \\
112 & 4.38363 & 0.0509397 & 0.0412051 & 10 & 4.50196 \\
113 & 4.42776 & 0.0520021 & 0.0450044 & 10 & 4.61596 \\
114 & 15 & 0.051641 & 0.0294849 & 10 & 4.46569 \\
115 & 10.1591 & 0.0515959 & 0.0324259 & 10 & 4.48113 \\
116 & 0.5 & 0.0515295 & 0.0299367 & 10 & 5.11227 \\
117 & 15 & 0.0531735 & 0.0377661 & 10 & 4.6232 \\
118 & 15 & 0.02256 & 0.0001 & 5.05418 & 0.0891896 \\
119 & 15 & 0.052929 & 0.0001 & 10 & 4.5906 \\
120 & 15 & 0.0527574 & 0.0183535 & 10 & 4.57485 \\
\hline Runs: 12 & & & & & Converged \\
\hline Total: 120 & & & & & Done \\
\hline
\end{tabular}

Table 11: Design variables and objective values for block iteration 10

Number of runs in block: 12

Block solving time: $3 \mathrm{~s}$

Total elapsed time: $108 \mathrm{~s}$

Total number of runs: 120 
$\mathrm{X} 2=0.0226, \mathrm{X} 3=0.0001, \mathrm{X} 4=3.9 \quad \mathrm{X} 1=3.3, \mathrm{X} 3=0.0001, \mathrm{X} 4=3.9$
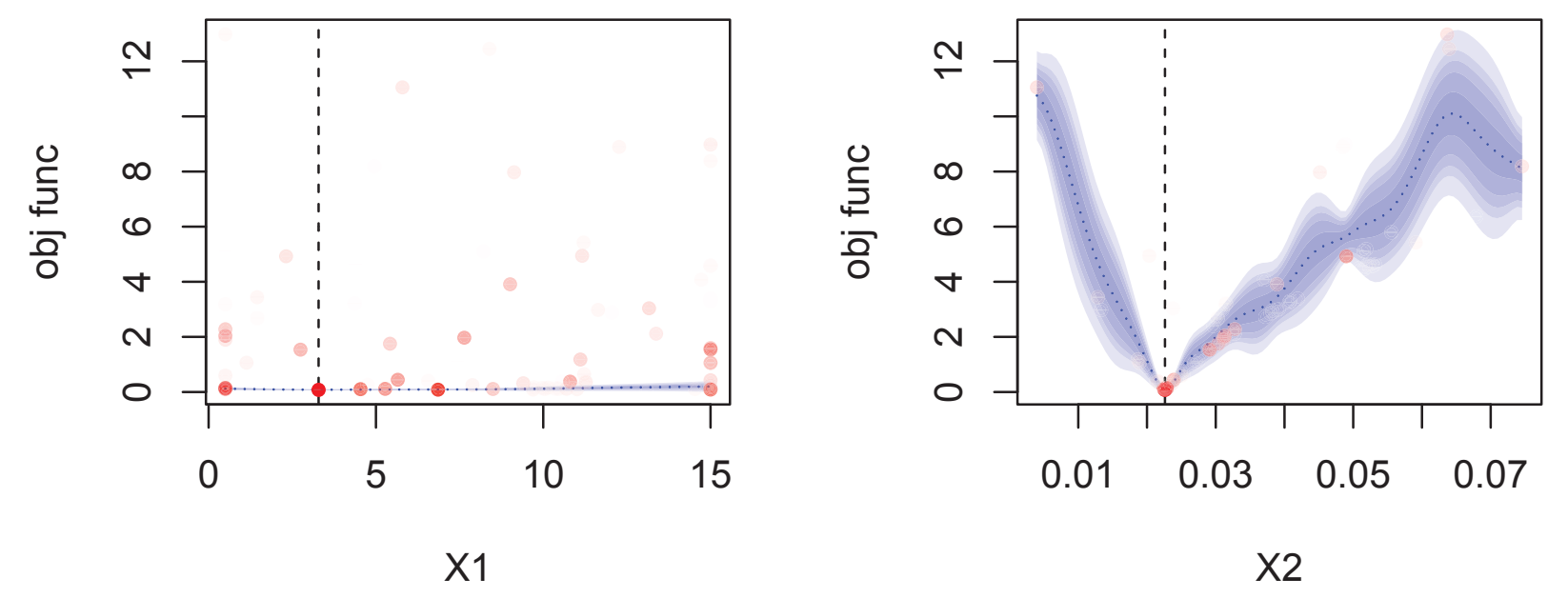

$\mathrm{X} 1=3.3, \mathrm{X} 2=0.0226, \mathrm{X} 4=3.9 \quad \mathrm{X} 1=3.3, \mathrm{X} 2=0.0226, \mathrm{X} 3=0.0001$
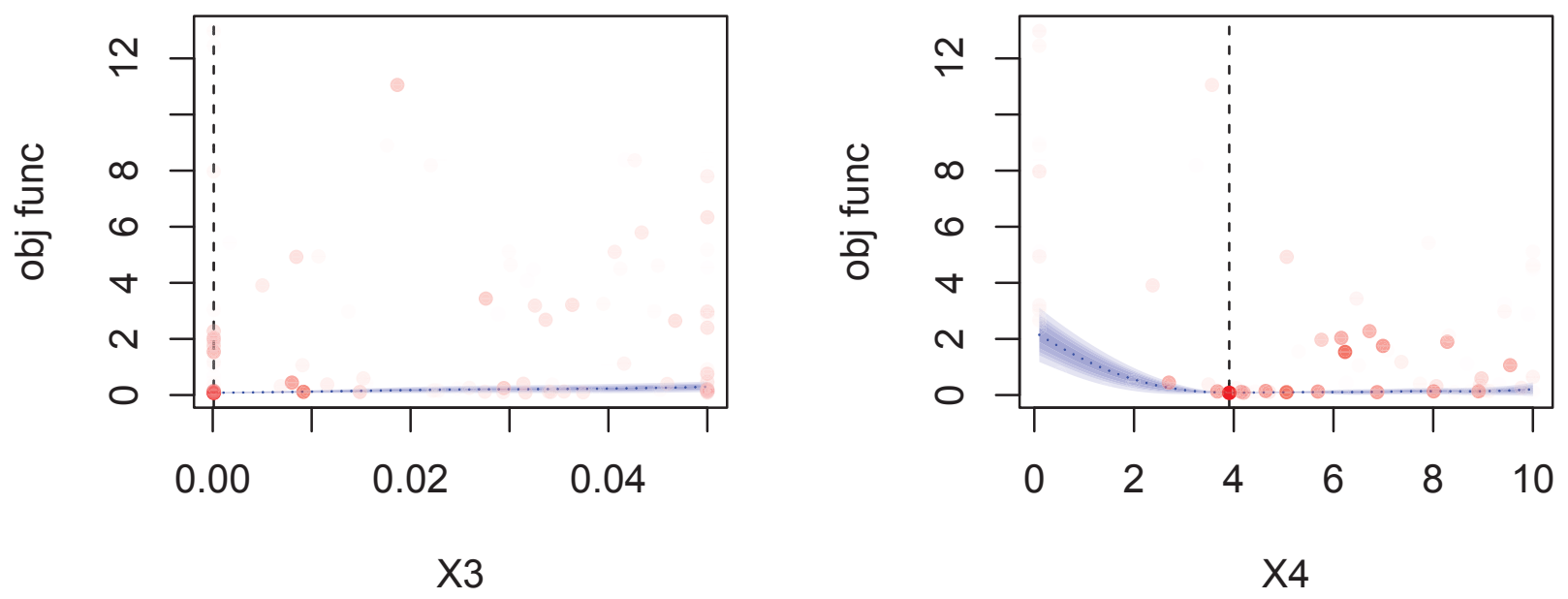

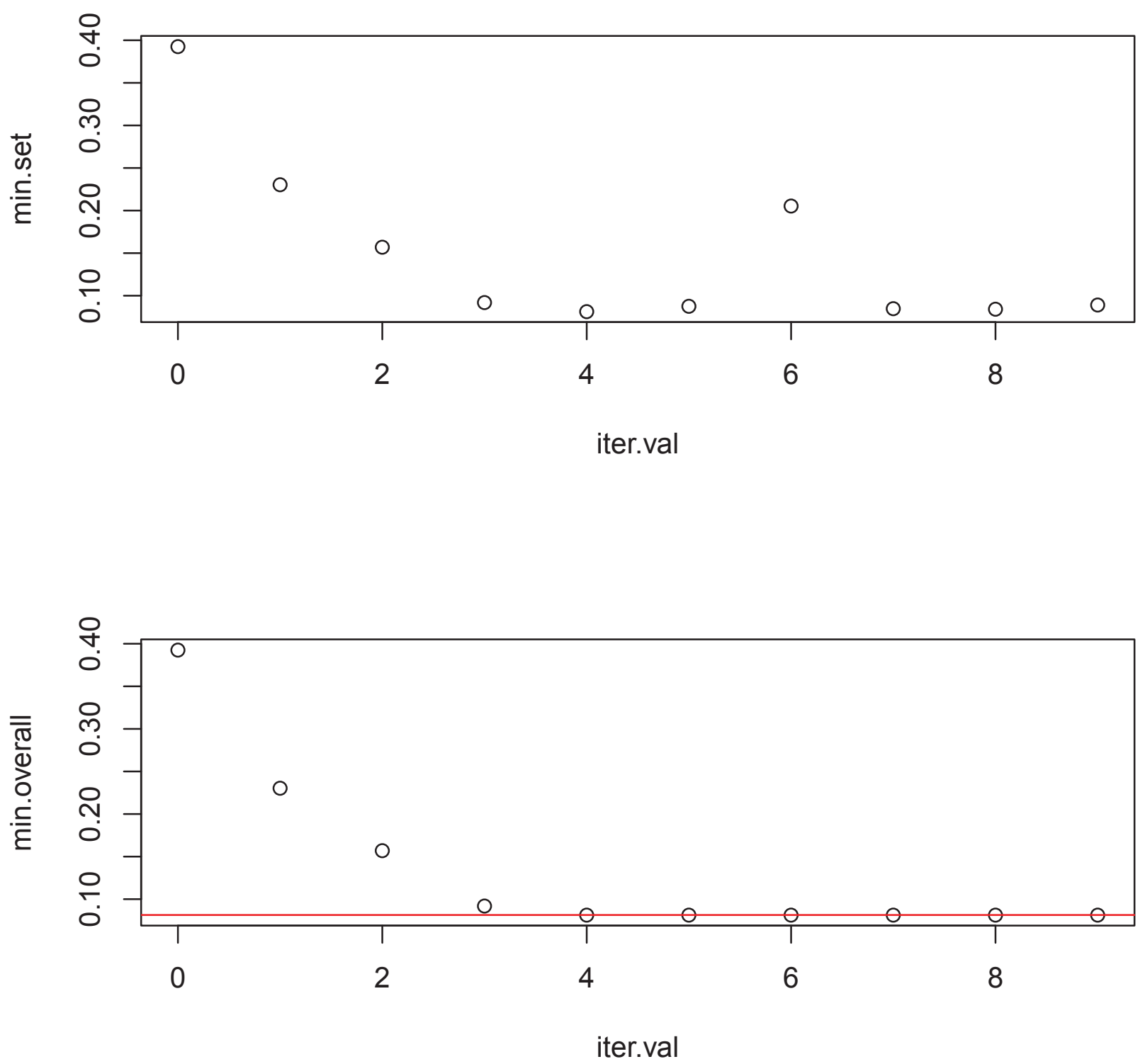